\title{
A CUSTOMIZABLE ARTIFICIAL AUDITORY FOVEA
}

by

\author{
Christopher Ness Casebeer
}

A dissertation submitted in partial fulfillment of the requirements for the degree

of

Doctor of Philosophy

in

Electrical Engineering

\section{MONTANA STATE UNIVERSITY}

Bozeman, Montana

November 2018 


\section{(c) COPYRIGHT}

by

Christopher Ness Casebeer

2018

All Rights Reserved 


\section{TABLE OF CONTENTS}

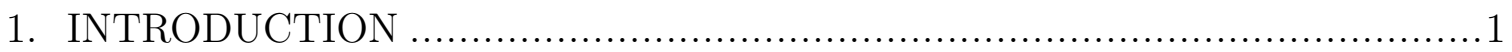

2. BIOLOGICAL INSPIRATION .................................................

Foveation in Vision .................................................................. 3

Auditory Foveation in Animals .................................................... 3

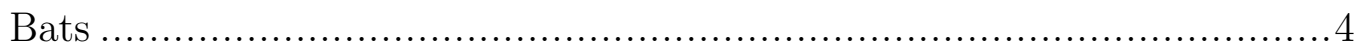

Barn Owls ..................................................................... 6

Marmoset Monkey Neurological Studies ............................................... 7

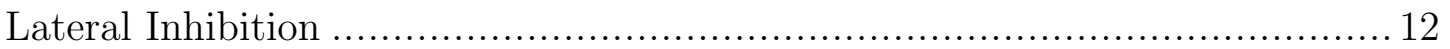

3. TIME-FREQUENCY ANALYSIS ........................................... 13

The Short Time Fourier Transform …............................................ 22

The Uncertainty Principle ................................................... 27

AM and FM Contributions to Bandwidth ....................................... 31

4. CUSTOM TILING OF THE TIME-FREQUENCY PLANE ....................... 33

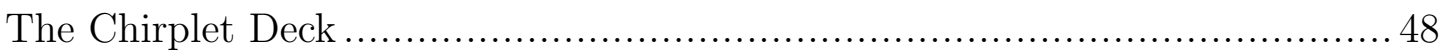

Custom Foveation of the Time-Frequency Plane ............................... 52

Distance Metric..................................................................... 55

5. SIGNAL PROCESSING USING THE ARTIFICIAL ACOUSTIC

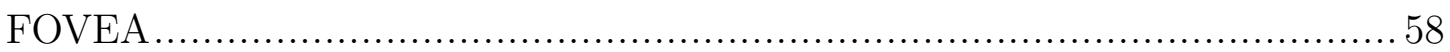

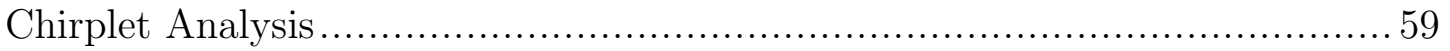

High Spatial Foveation...................................................... 62

Chirplet Deck Analysis........................................................... 65

High Deck Foveation.................................................... 67

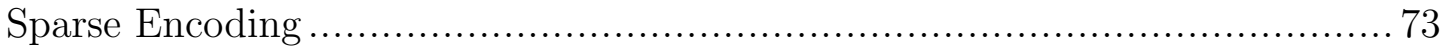

6. ARTIFICIAL NEURAL NETWORKS ........................................... 88

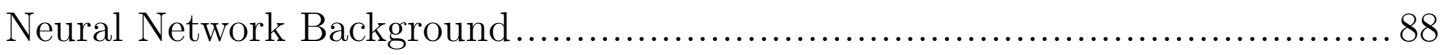

Convolutional Neural Networks and TensorFlow .................................. 95

Convolutional Network Configuration........................................ 97

7. USING THE CUSTOMIZABLE ARTIFICIAL AUDITORY FOVEA IN MACHINE LEARNING 


\section{TABLE OF CONTENTS - CONTINUED}

Chirplet Decks Used for the Front End ...................................... 101

8. ARTIFICIAL AUDITORY FOVEA MATLAB CODE.......................... 110

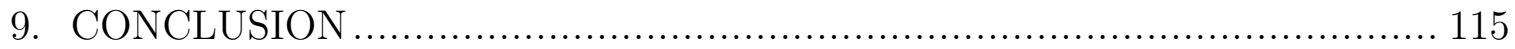

Future Directions................................................................... 115

REFERENCES CITED .................................................... 120

APPENDIX: Matlab artificial accousic fovea chirp and deck creation .............. 124 


\section{LIST OF TABLES}

Table

5.1 Example foveation. Very low spatial and low deck foveation.............60 60

5.2 Example foveation. High spatial foveation and low deck foveation. ............................................................ 64

5.3 Example foveation. High spatial foveation and high

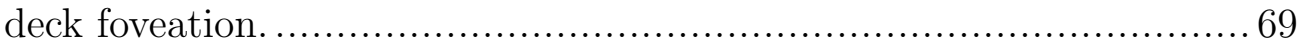

7.1 High deck resolution high spatial resolution. ........................... 101

7.2 Low deck resolution high spatial resolution. ........................... 102

7.3 High deck resolution low spatial resolution............................ 102 


\section{LIST OF FIGURES}

Figure

Page

2.1 High density of cone cells create a fovea in the back of the human eye [42].

2.2 P. Parnelli neural study. Note the large $61-62 \mathrm{kHz}$ processing area as well as the number of neurons in this area dedicated to $61-62 \mathrm{kHz}[34]$.

2.3 P. Parnellie primary echolocation signal. Note the

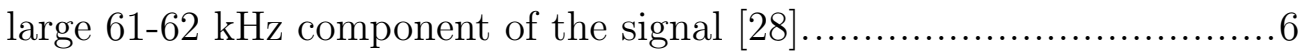

2.4 A larger proportion of barn owl cochlear hair cells are dedicated to behaviorally relevant sound creating an auditory fovea [35].

2.5 Tonotopic organization of Marmoset auditory cortex [13] ................. 9

2.6 Marmoset auditory neuron differentiating modulation and onset [20].

2.7 Marmoset auditory neuron differentiating signal onset pattern [21].

3.1 The square wave begins to take shape as we add more component sinusoids.

3.2 The sinusoids making up a square wave. 14

3.3 Windowing prior to Fourier transform. ................................. 16

3.4 Spectrogram. ......................................................... 17

3.5 Fourier transform of infinite sinusoid. ............................... 18

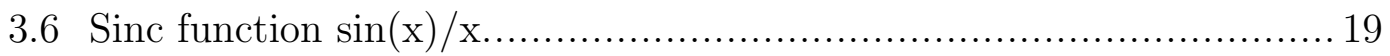

3.7 Varying window lengths and their Fourier transforms. ...................20

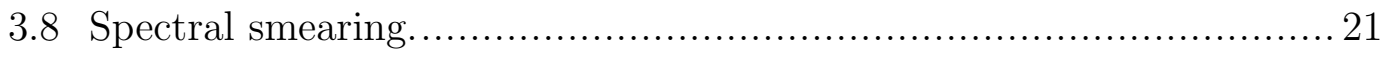

3.9 Windowing prior to Fourier transform. ................................... 23 


\section{LIST OF FIGURES - CONTINUED}

Figure

3.10 Wideband/narrowband spectrogram. The spectrogram window sizes used. Narrowband: .1 Seconds. Balanced: .005 Seconds. Wideband: .0005 Seconds. Evaluated every 25 samples.

3.11 Narrowband spectrogram time-frequency tiling. 25

3.12 Wideband spectrogram time-frequency tiling. 26

3.13 Infinite bandwidth through single point in time. 27

3.14 Illustration of duration and bandwidth of signal.

3.15 Gaussian modulated sinusoid.

4.1 Marmoset twitter call and proposed tiles. Balanced spectrogram : .005 second window size.

4.2 Chirp with no FM and tiling. Chirp is centered at $1000 \mathrm{~Hz}$ with a $0 \mathrm{~Hz} / \mathrm{sec}$ slope.

4.3 Upward chirp. Chirp is centered at $1700 \mathrm{~Hz}$ with a $235 \mathrm{kHz} / \mathrm{sec}$ slope.

4.4 Downward chirp. Chirp is centered at $1700 \mathrm{~Hz}$ with a $-235 \mathrm{kHz} / \mathrm{sec}$ slope. .36

4.5 Two chirplets under differing envelopes....

4.6 Linear chirp and tiling. Onset modulation. Chirp is centered at $1000 \mathrm{~Hz}$ with a $0 \mathrm{~Hz} / \mathrm{sec}$ slope.

4.7 Upward chirp. Onset modulation. Chirp is centered at $1700 \mathrm{~Hz}$ with a $235 \mathrm{k} 5 \mathrm{~Hz} / \mathrm{sec}$ slope.

4.8 Downward chirp. Onset modulation. Chirp is centered at $1700 \mathrm{~Hz}$ with a $-235 \mathrm{kHz} / \mathrm{sec}$ slope.

4.9 Linear chirp and tiling. Offset modulation. Chirp is centered at $1000 \mathrm{~Hz}$ with a $0 \mathrm{~Hz} / \mathrm{sec}$ slope.

4.10 Upward chirp. Offset modulation. Chirp is centered at $1700 \mathrm{~Hz}$ with a $235 \mathrm{kHz} / \mathrm{sec}$ slope. 
vii

\section{LIST OF FIGURES - CONTINUED}

Figure

Page

4.11 Downward chirp. Offset modulation. Chirp is centered at $1700 \mathrm{~Hz}$ with a $-235 \mathrm{kHz} / \mathrm{sec}$ slope.

4.12 A comparison of alternating contributions from either amplitude modulation or frequency modulation. In $\mathrm{A}$, a low alpha parameter compared to $\mathrm{B}$ creates a lower AM contribution. In $\mathrm{C}$ a lower Beta parameter compared to D creates a lower FM contribution to bandwidth.

4.13 Illustration of different signals which all have identical bandwidth but varying levels of AM and FM contributions to bandwidth [10].

4.14 Arbitrary control of time-frequency tiling. Placement of the chirplets could be anywhere, but chirplets of similar rotations are grouped together to show varying window lengths.

4.15 Visualization of tiling the time-frequency plane with chirplet decks. ...

4.16 Slope and length chirplet deck parameters.

4.17 Time and frequency step chirplet deck parameters. Each yellow dot represents a chirplet deck as was the case in Figure 4.15.

4.18 High and low spatial foveation time frequency plane. Each point in the figure portrays a chiplet deck center evaluation point. On the left we evaluate much more finely in time and frequency.

4.19 High foveation of chirplet deck. Here we see multiple chirplet lengths and slopes comprising high deck foveation.

4.20 Lower foveation of chirplet deck. The chirplet deck does not have as many lengths or slopes, comprising low deck foveation. 
viii

\section{LIST OF FIGURES - CONTINUED}

Figure

Page

4.21 A continuous time chirp signal is discretely sampled for the Matlab implementaiton of the artificial accousic fovea. 57

5.1 Translation of the chirplet deck in time and frequency.

5.2 Matlab structure of chirplet deck parameters.

$5.3-1100 \mathrm{~Hz} / \mathrm{sec}$ sloping chirplet .005 seconds match. Low spatial foveation. Chirplet deck parameters are shown in Table 5.1. The spectrogram window used was: Balanced: .005 Seconds.

$5.4-1100 \mathrm{~Hz} / \mathrm{sec}$ sloping chirplet .005 seconds match. High spatial foveation. Chirplet deck parameters are shown in Table 5.2. The spectrogram window used was: Balanced: .005 Seconds.

$5.5-1100 \mathrm{~Hz} / \mathrm{sec}$ sloping chirplet .005 seconds match. High spatial foveation closer. Chirplet deck parameters are shown in Table 5.2. The spectrogram window used was: Balanced: .005 Seconds.

5.6 Overall best chirp output for high spatial foveation and low deck foveation. Chirplet deck parameters are shown in Table 5.2. The spectrogram window used was: Balanced: .005 Seconds.

5.7 Overall best chirp output for high spatial foveation and low deck foveation zoomed. Chirplet deck parameters are shown in Table 5.2. The spectrogram window used was: Balanced: .005 Seconds.

5.8 Overall best chirp output for a high deck foveation and high spatial foveation. Chirplet deck parameters are shown in Table 5.3. The spectrogram window used was: Balanced: .005 Seconds. 


\section{LIST OF FIGURES - CONTINUED}

Figure

Page

5.9 Overall best chirp output zoom for a higher deck foveation and high spatial foveation. Chirplet deck parameters are shown in Table 5.3. The spectrogram window used was: Balanced: .005 Seconds.

5.10 Constant chirp match results. Chirplet deck parameters are shown in Table 5.3. The spectrogram window used was: Balanced: .005 Seconds.

5.11 All chirp match results against a Marmoset phee call. Chirplet deck parameters are shown in Table 5.3. The spectrogram window used was: Balanced: .005 Seconds.

5.12 All chirp match results against a Marmoset twitter call. Chirplet deck parameters are shown in Table 5.3. The spectrogram window used was: Balanced: .005 Seconds

5.13 Best chirp match to signal across all frequencies and chirp decks for an instant in time. We see the longer chirps represented by the 400 indicators matching the signal less often.

5.14 Best chirp in window separation..................................... 76

5.15 Best chirp in window separation..................................... 77

5.16 Best chirplet match identifcation subplot. The bottom subplot shows which type of chirp is best matching the signal at that particular point in time and frequency. An inlay graphic shows the corresponding color code of chirplet types. Chirplet deck parameters are shown in Table 5.1. The spectrogram window sizes used. Narrowband: .1 Seconds. Balanced: .005 Seconds. Wideband: .0005 Seconds. 


\section{LIST OF FIGURES - CONTINUED}

Figure

Page

5.17 Best chirplet match identifcation subplot. The bottom subplot shows which type of chirp (out of 38 types) is best matching the signal at that particular point in time and frequency. An inlay graphic shows the corresponding color coding of chirplet types. Chirplet deck parameters are shown in Table 5.3. The spectrogram window sizes used. Narrowband: .1 Seconds. Balanced: .005 Seconds. Wideband: .0005 Seconds

5.18 Best chirplet match identifcation subplot. The bottom subplot shows which type of chirp (out of 38 types) is best matching the signal at that particular point in time and frequency. An inlay graphic shows the corresponding color coding of chirplet types. Chirplet deck parameters are shown in Table 5.3. The spectrogram window sizes used. Narrowband: .1 Seconds. Balanced: .005 Seconds. Wideband: .0005 Seconds

5.19 Illustration of an experimental visualization inlay graphic. This is an example of the number of sloping chirplets each with different color code for identification plots....

5.20 This figure shows a zoomed in version of the marmoset twitter call. We are looking at a particular phrase. The blue color of the bottom figure represents onset type chirplets. The yellow color represents offset type chirplets. We can see that much more onset types are being activated at the onset of a phrase. This is illustrative and what is expected.

5.21 A Marmoset trill call wherein the fovea is not customized with higher spatial resolution on a frequency axis band. Chirplet deck parameters are shown in Table 5.3. No additional chirplets are matched in the $6000-8000 \mathrm{~Hz}$ band beyond the default $500 \mathrm{~Hz}$ steppings. 


\section{LIST OF FIGURES - CONTINUED}

Figure

5.22 A Marmoset trill call wherein the fovea is customized with higher spatial resolution on the frequency axis in a frequency band. Chirplet decks are stamped more tightly along the frequency axis. Chirplet deck parameters are shown in Table 5.3. Additional chirplets are matched in the $6000-8000 \mathrm{~Hz}$ band at $10 \mathrm{~Hz}$ steps.

6.1 The neural network neuron (perceptron) ............................... 90

6.2 Fully connected neural network connections........................... 91

6.3 Example of machine learning network arrangements [41]................ 94

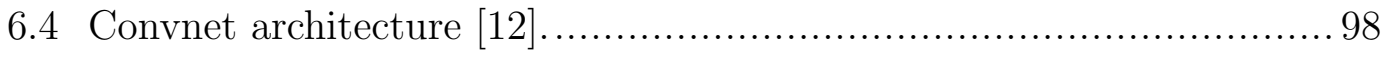

7.1 Training accuracy of chirplet input network on training set over all independent training runs. Each line represents an independent training of the network from start.

7.2 Cost function reduction of chirplet input network on training set over all independent training runs. Each line represents an independent training of the network from start.

7.3 Accuracy of chirplet input network on unseen test set data over all independent training runs. Each line represents an independent training of the network from start. Test set accuracy indicates ability of model to generalize.

7.4 Comparison of average training set accuracy across different front ends. Each line is the average of 50 independent trainings of the network on the indicated front end data.

7.5 Comparison of average cost function reduction across different front ends. Each line is the average of 50 independent trainings of the network on the indicated front end data. 


\section{LIST OF FIGURES - CONTINUED}

Figure

7.6 Comparison of test set accuracy different front ends. Each line is the average of 50 independent trainings of the network on the indicated front end data.......................... 109

9.1 Histogram of single chirplet response. ................................ 117

9.2 Cumulative distribution function of a particular chirp. ................ 118 


\begin{abstract}
We, as humans, can separate and attend to audio sources in mixtures of sounds and noise. We can listen distinctly to a friend at a party in a sea of background noise and conversations. Human auditory neurology exceeds even state-of-the-art audio algorithms. How are we able to do this? This dissertation takes inspiration from biology to frame a novel audio processing front-end. Neurobiology shows that auditory neurons isolate signal onsets, timing, frequency, amplitude and modulation characteristics. Why is it then that many standard processing methods choose to ignore this information or make the assumption that machine learning will extract it regardless of input processing? This dissertation uses time-frequency analysis principles towards building a new front-end aimed at preserving these fine temporal and spectral details of the original signal to improve audio system detection and recognition. The system allows keeping the fine frequency and time characteristics of a signal during analysis, while allowing customization of how much and where this resolution is kept. Like biology, this front-end can dedicate resources to detecting important signal events. It can over represent or foveate regions of the time-frequency plane that are important to the signal processing task at hand. These fine details are hypothesized to help enable audio learning algorithms to detect the fine nuances that distinguish musical instruments, determine the characteristics of a specific persons voice, or even detect the emotional state of a person. This customizable auditory fovea aims to mimic the powerful detection capability found in biology which is in contrast to standard methods in audio signal processing.
\end{abstract}




\section{INTRODUCTION}

Biology and the capability of the human body is the ultimate existence proof. The human brain does amazing cognitive tasks which computers can not replicate. We as humans can learn new concepts and our brains remain plastic towards learning new concepts. The human visual system can discern occluded objects or objects which have never been seen in the viewed context before. In a similar fashion, the human auditory system far outpaces even state of the art audio and speech recognition systems. We can focus our attention on auditory objects, such as focusing on one conversation in a sea of other signals and noise $[6,7,9]$. A person can also tease apart and attend to one auditory object in a mixture of objects. Another existence proof is our ability to attend to speech contained in background talking [9]. Biology has developed in such a way to allow us to excel at these tasks. This dissertation focuses on taking inspiration from biology to create a biologically inspired audio processing front end. This front end is framed by the fields of signal processing, time-frequency analysis and information theory to ground the project. Each of these frameworks are examined and explained how they are used. Current methods in speech and audio recognition can destroy critical pieces of a signal's fine information. A front end in this context is the portion of a computer algorithm which preprocesses a signal before it is used by other parts of the algorithm. It is the crucial representation step in machine learning. It is our belief that a front end which is inspired by biology

and framed in engineering could be of great use as a biomimetic method in audio recognition since biology has already solved the representation problem. It is the aim of this project to create a new front end for audio and speech recognition. This 
dissertation will examine the biological inspiration for the front end, time-frequency analysis tenets which frame the front end, how the front end is assembled, how the back end is assembled and the results when the processing pipeline is tested. 


\section{BIOLOGICAL INSPIRATION}

\section{$\underline{\text { Foveation in Vision }}$}

The biology of human and animal brains shows us informative concepts which can guide our path. The concept of a visual fovea is well known. The density of cones in the retina of the eye peaks as we approach the center of the back of the eye $[5,33,42]$. This foveation gives us high acuity in our central vision. We lose acuity as we move away from the center of vision. The high acuity is due to a higher dedication of resources,i.e cone cells, then what is found in peripheral vision. The cone cells reach peak density in the fovea of the human eye shown in Figure 2.1.

(a)

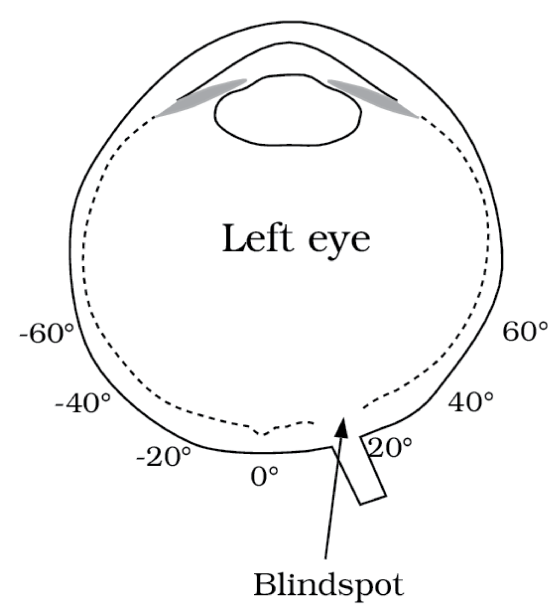

(b)

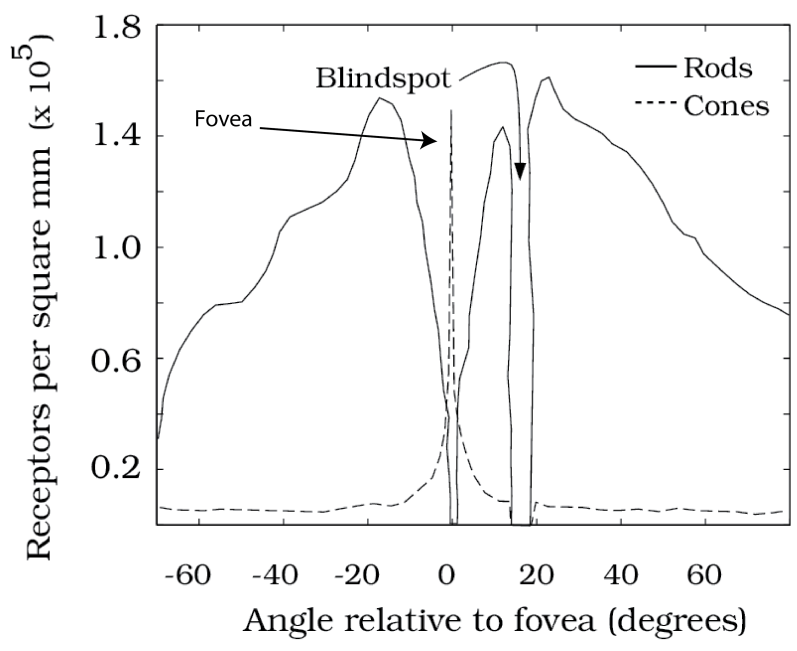

Figure 2.1: High density of cone cells create a fovea in the back of the human eye [42].

\section{Auditory Foveation in Animals}

Similar to visual foveation, audio fovea structures have been shown in animals. Like the retina, these auditory foveae allow bats to attend to specific characteristics 
of incoming audio in better detail. In bats, it has been shown that an auditory or acoustic fovea is evident $[28,34]$.

Bats

A species of bat, P. Parnelli, has been studied and shown to exhibit neural structures dedicated to processing its own echo location signal. The neural study shown in Figure 2.2 shows a large "flutter processing area" which conveniently has a large number of neurons dedicated to $61 \mathrm{kHz}$ processing. One of P. Parnelli's primary calls correspondingly has a large $61 \mathrm{kHz}$ component as shown in Figure 2.3. 

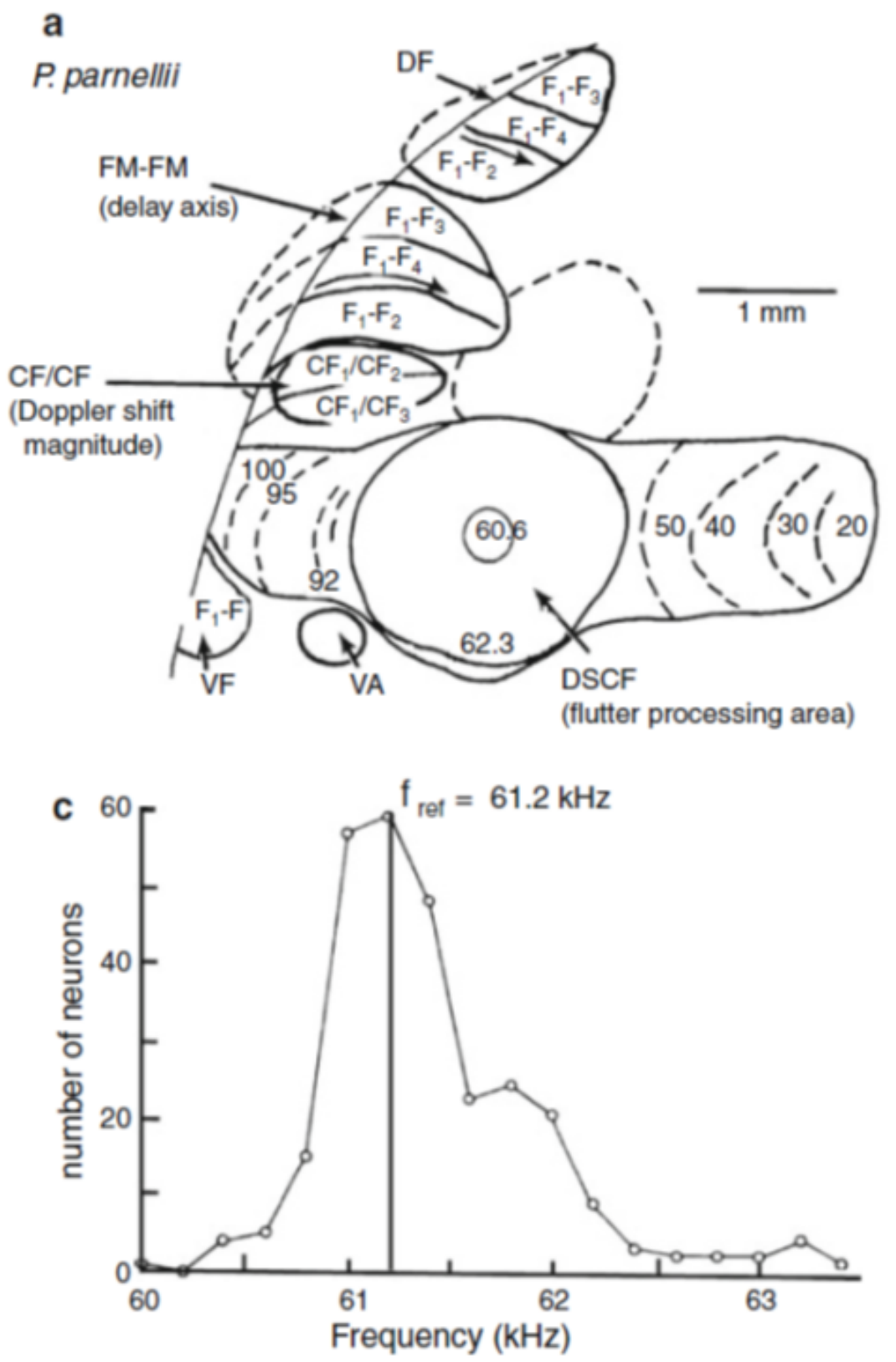

Figure 2.2: P. Parnelli neural study. Note the large $61-62 \mathrm{kHz}$ processing area as well as the number of neurons in this area dedicated to $61-62 \mathrm{kHz}$ [34]. 

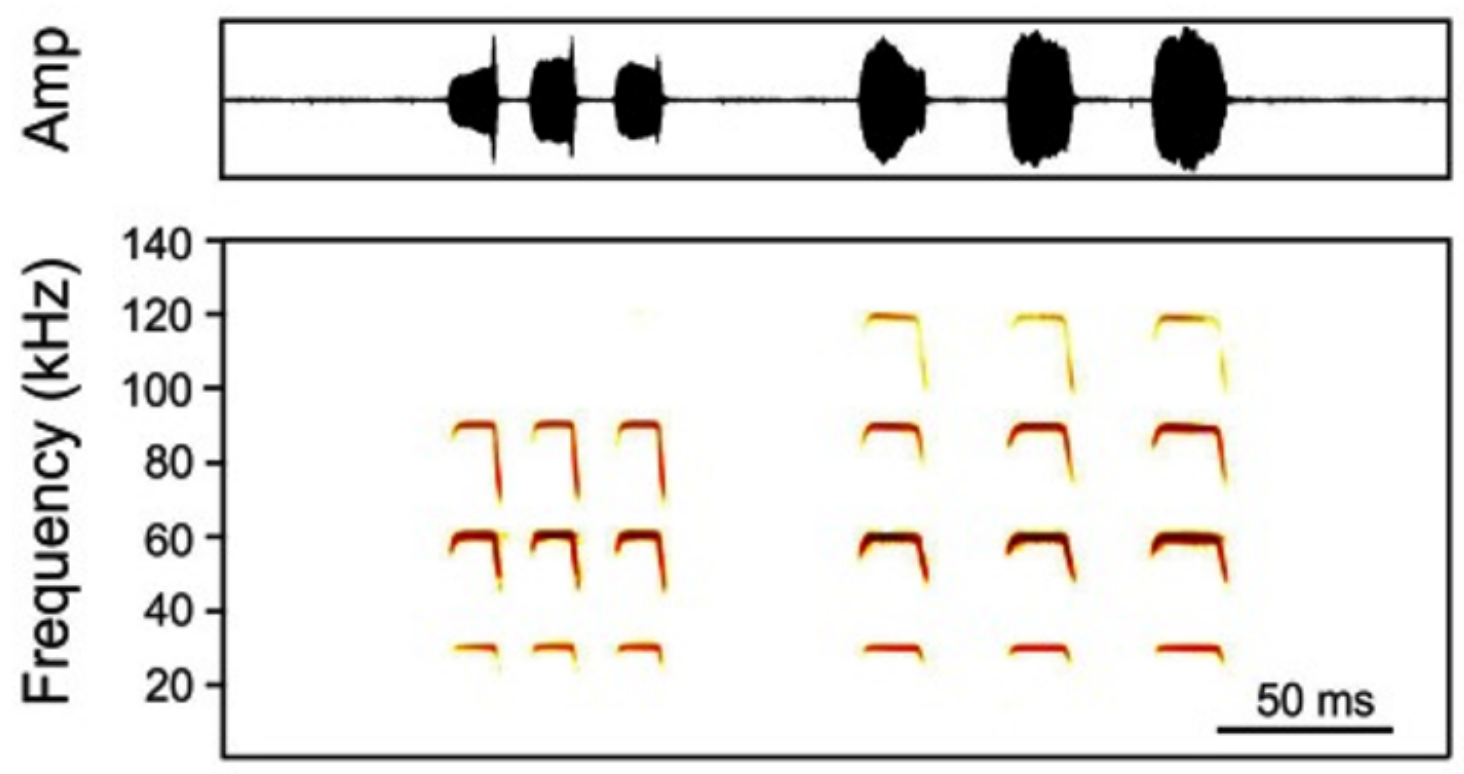

Figure 2.3: P. Parnellie primary echolocation signal. Note the large $61-62 \mathrm{kHz}$ component of the signal [28].

Similarly, a portion of the horseshoe bat's basilar membrane has a large portion dedicated to sensing the pure tone component of its own echo location signal [35]. Resources are being dedicated to signals which are important for the species.

\section{Barn Owls}

Owls are another species with an auditory fovea [19]. The auditory fovea of the barn owl occurs in the basilar membrane inside the cochlea, a primary animal structure responsible for the transduction of air pressure waves into electrical stimulus. An overrepresentation of hair cells dedicated to behaviorally relevant frequencies is found. This overrepresentation is shown in Figure 2.4. It is this overrepresentation that is taken as inspiration to create an overrepresentation of detectors in a front end system for acoustic analysis. If a portion of signal is of great importance in detection or a portion of a signal is characteristic of something we want to detect and analyze, biology inspires us to dedicate more resources in areas that need finer detection. Note 
also that foveation can occur at any layer of neural processing. Foveation has been shown in the ear of owls or in the auditory cortex of bats.

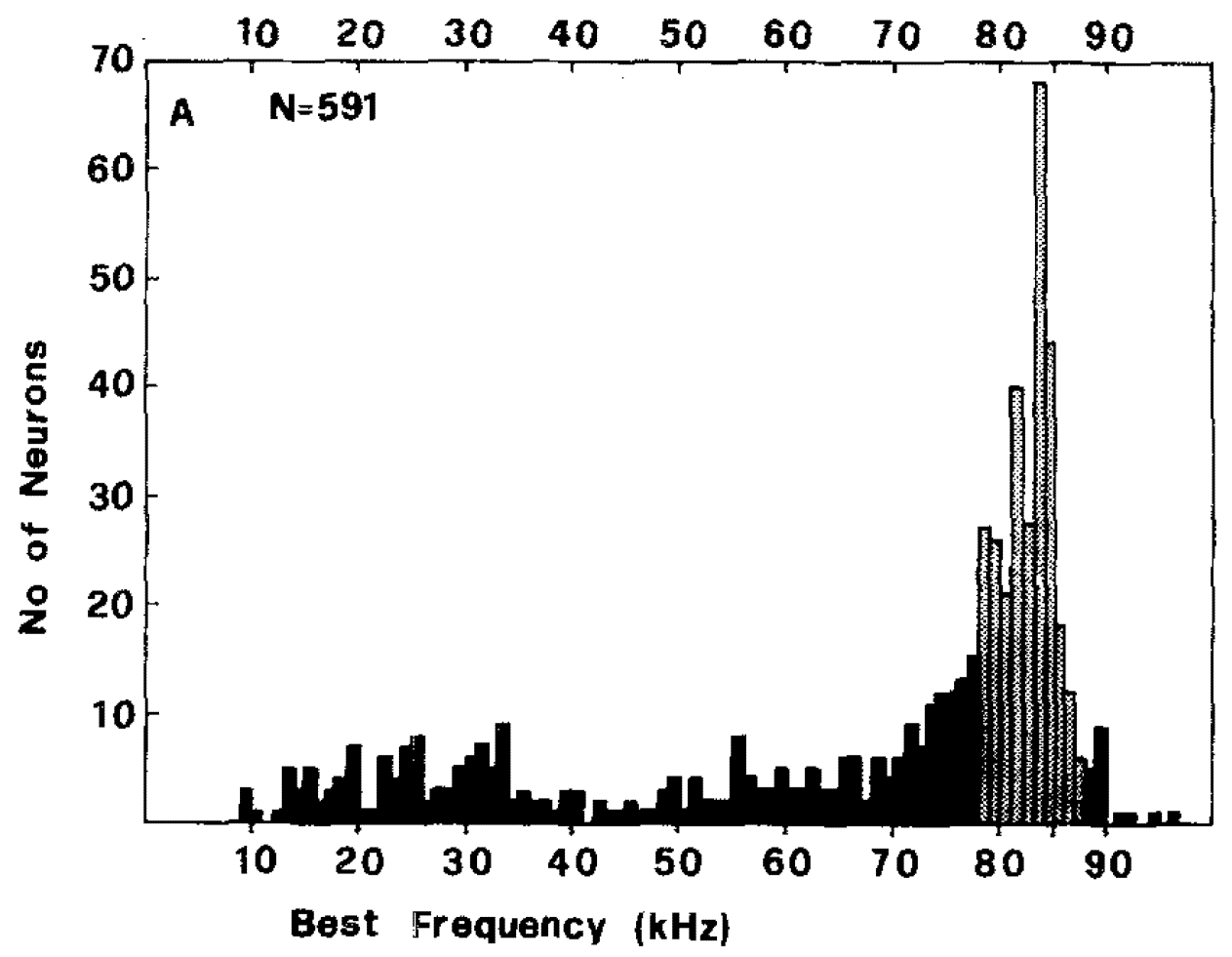

Figure 2.4: A larger proportion of barn owl cochlear hair cells are dedicated to behaviorally relevant sound creating an auditory fovea [35].

\section{Marmoset Monkey Neurological Studies}

Another inspiration is the study of the Marmoset monkey, a primate animal neurology model $[18,27]$. This animal model has fine neural auditory sensitivity to many elements of a signal. Neurons and areas of the Marmoset auditory cortex have been found to respond to specific frequency and specific temporal patterns. Certain neurons fire only upon the initial onset of a test signal [21]. They are marking 
the accurate beginning of a signal. Certain neurons only fire when an incoming signal contains certain amplitude modulations [20]. Neurons are typically frequency selective [13]. This informs us, that the primate brain is paying attention to both frequency and time information. This is a biological reminder that resources are dedicated to pay careful attention to time, frequency, and amplitude modulation. A biologically inspired front end should finely monitor each of these elements. 


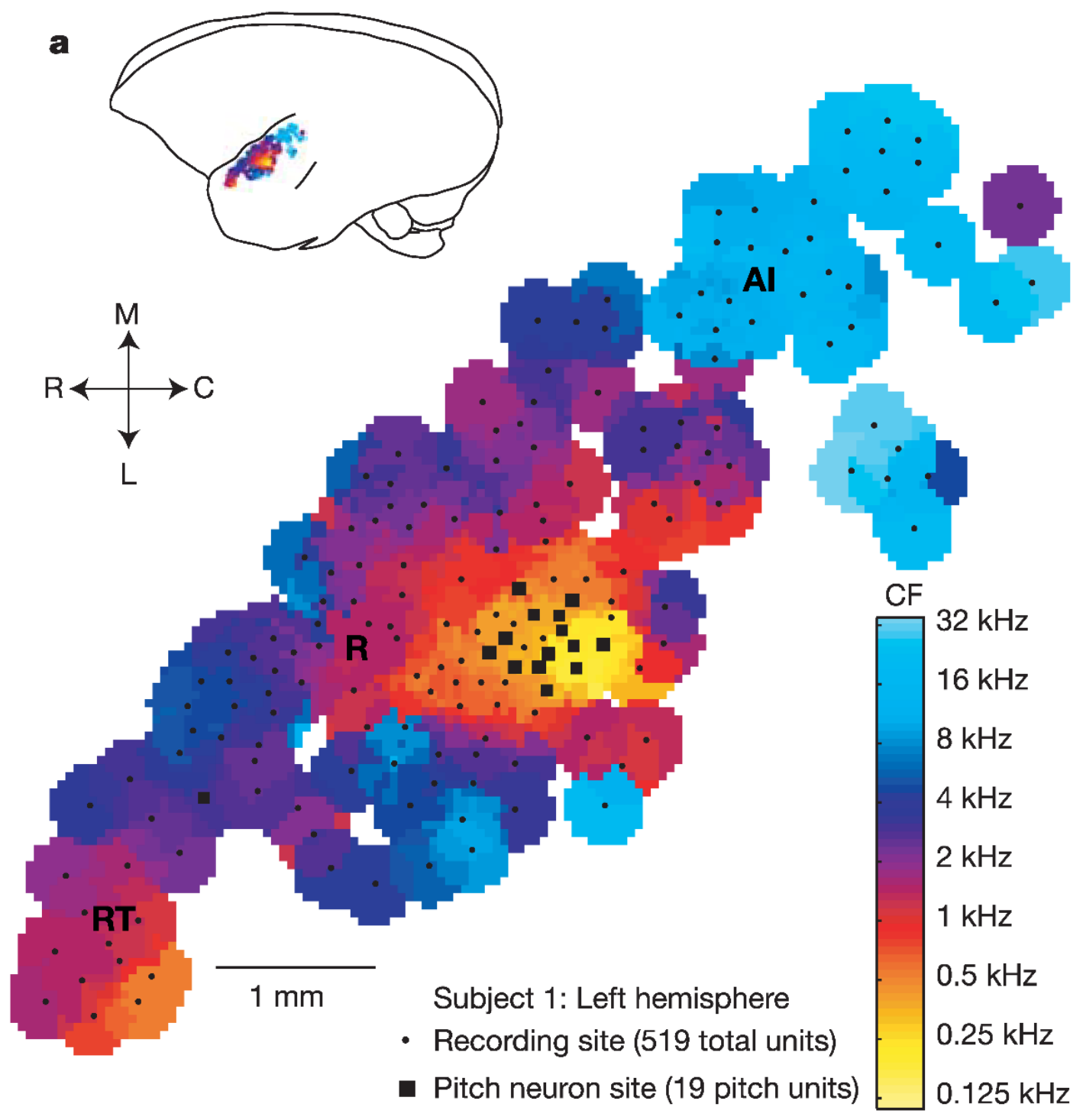

Figure 2.5: Tonotopic organization of Marmoset auditory cortex [13]. 
sAM

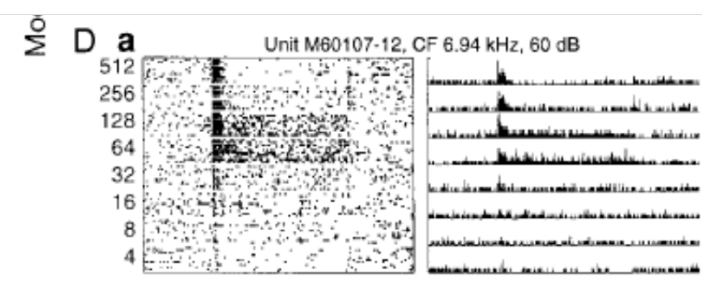

$\overline{\text { Time (ms) }}$
sFM

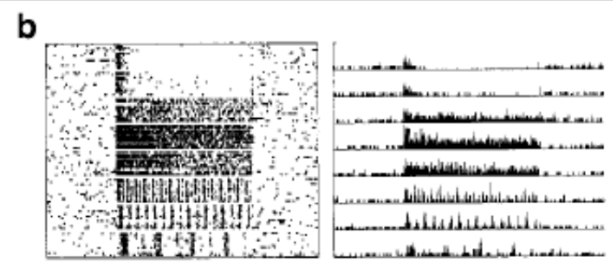

Time (ms)

Figure 2.6: Marmoset auditory neuron differentiating modulation and onset [20].

Figure 2.5 displays the frequency organization of the Marmoset auditory cortex [13]. Specific neurons respond best to specific frequencies. Figure 2.6 shows one neuron responding to two types of modulated stimuli [20]. The response patterns are markedly different. It is also important to note that the neurons begin firing very close in time to signal onset. This is evidence that neurons are marking the beginning of signals with great precision and pay attention to modulation patterns. The pattern of onset or attack of a signal has also been shown to differentiate neuron response in Marmoset auditory cortex [21]. This is shown as the different neuron (unit) responses to two different amplitude attack patterns of a signal shown in Figure 2.7. 

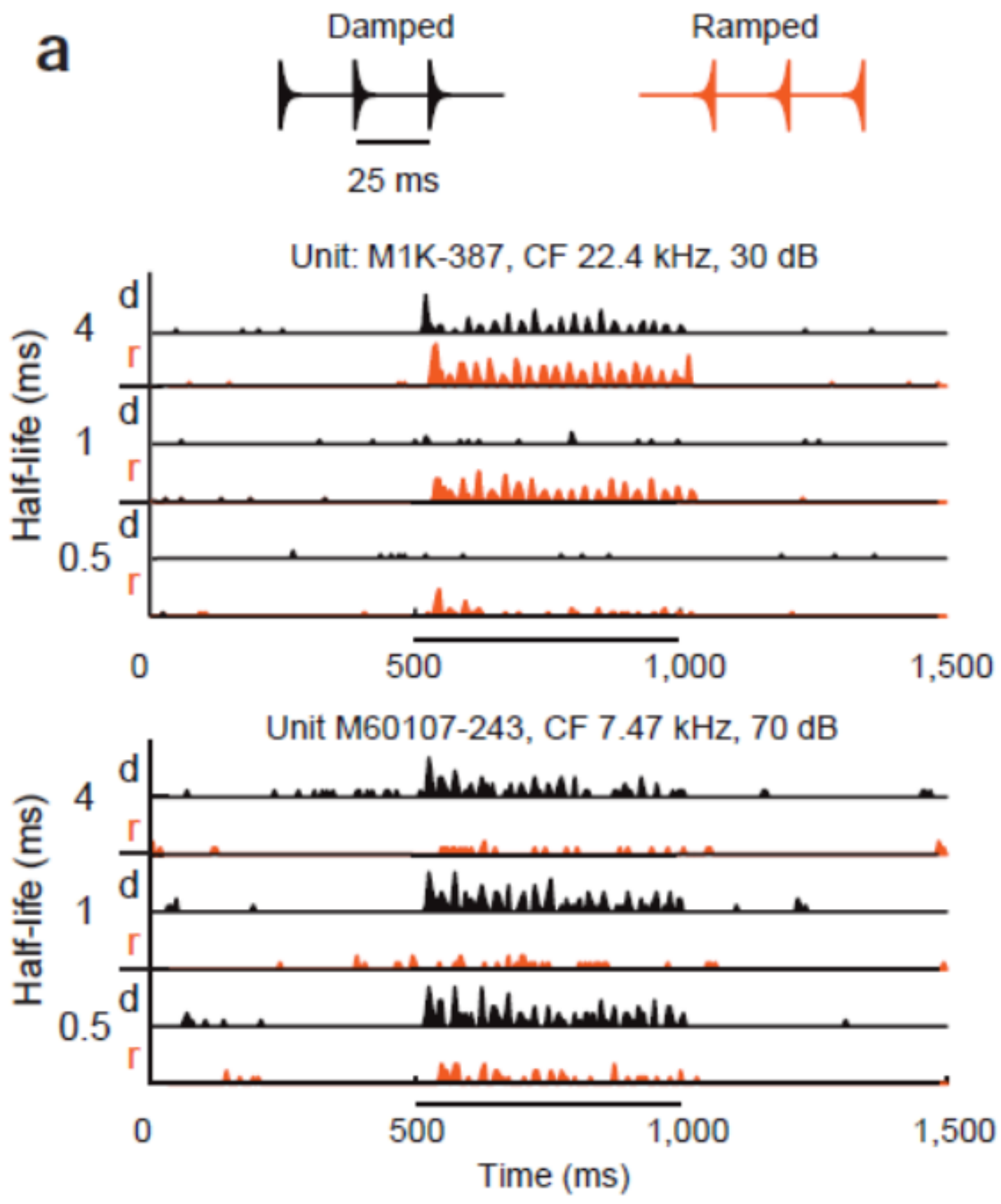

Figure 2.7: Marmoset auditory neuron differentiating signal onset pattern [21]. 


\section{Lateral Inhibition}

Another idea from biology which will filter into the dissertation is that of lateral inhibition. Lateral inhibition describes a decrease in a neuron's response to a stimulus when its neighbors, which match the signal better, are also stimulated [20]. Lateral inhibition has been found in visual, tactile and auditory systems across species. In essence, only the best neuron is allowed to fire in a bed of neurons when all neurons are stimulated similarly. This biological idea will inspire how the audio framework proposed will process its data and will be detailed. A nonlinear max operator is introduced in this project to sparsify data from the front end and will be talked about later in the dissertation to implement sparse coding.

This evidence shows us that if we are to use biology as a proof of concept that speech and audio recognition can be done, it can be done well and it can be done in noise, some part of our speech and audio algorithm should pay special attention to time, frequency and amplitude characteristics of a signal. An overrepresentation of detectors in the front end of the sensory systems has been used in biology successfully. These inspirations factor into the front end detailed in later sections. These ideas are in contrast to standard approaches to audio detection front ends where for example the spectrogram is used as the input to machine learning algorithms which have been proven brittle in auditory scene analysis. [4,16, 17, 29,39]. 


\section{TIME-FREQUENCY ANALYSIS}

A very common signal processing technique is the use of the Fourier transform. It is a mathematical transform which decomposes a signal into its component sinusoids [31]. The idea is that any signal can be built from a summation of many sinusoids of varying frequencies and phase shifts. For example, a square wave can be constructed from component sinusoids as shown in Figures 3.1 and 3.2.

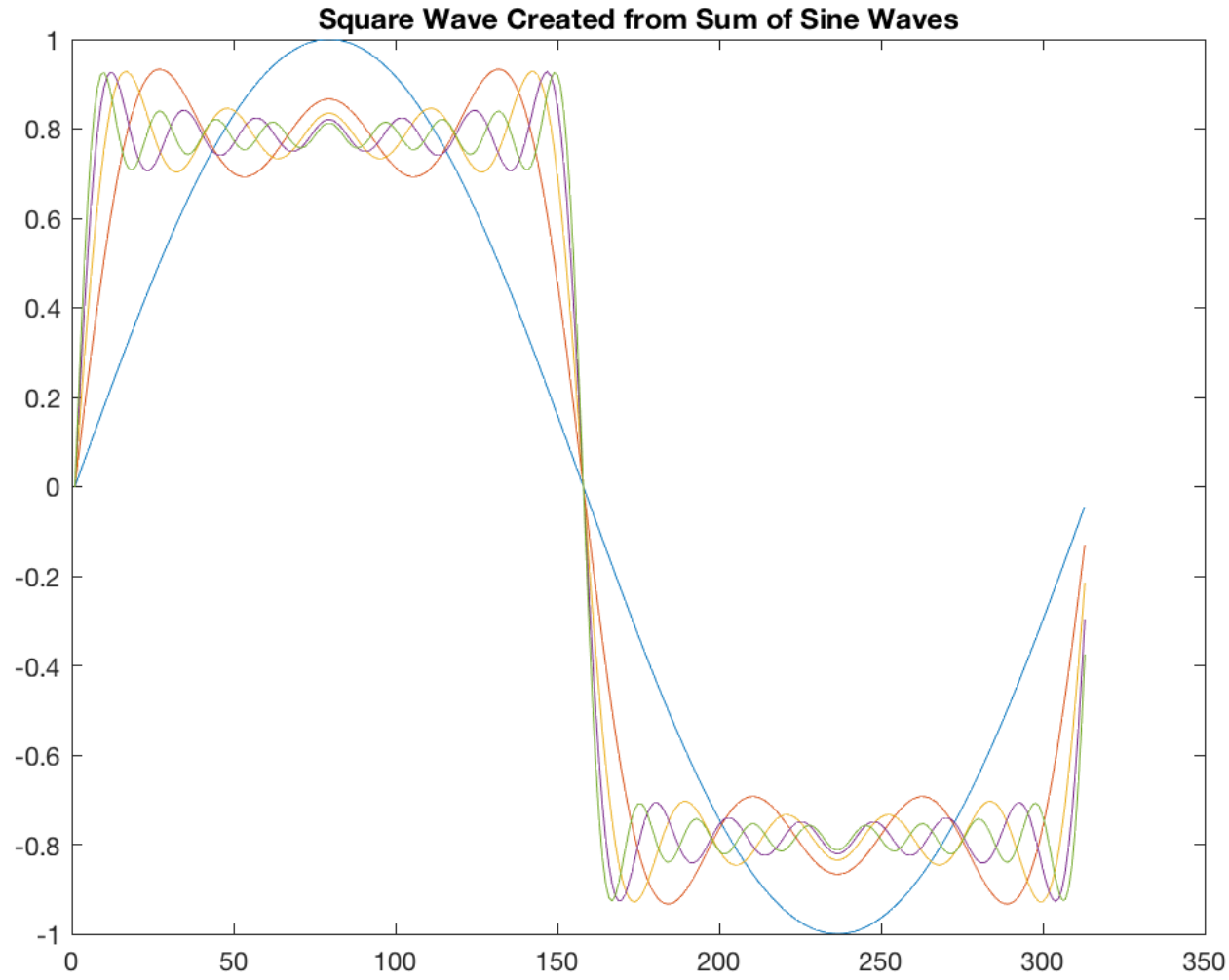

Figure 3.1: The square wave begins to take shape as we add more component sinusoids. 


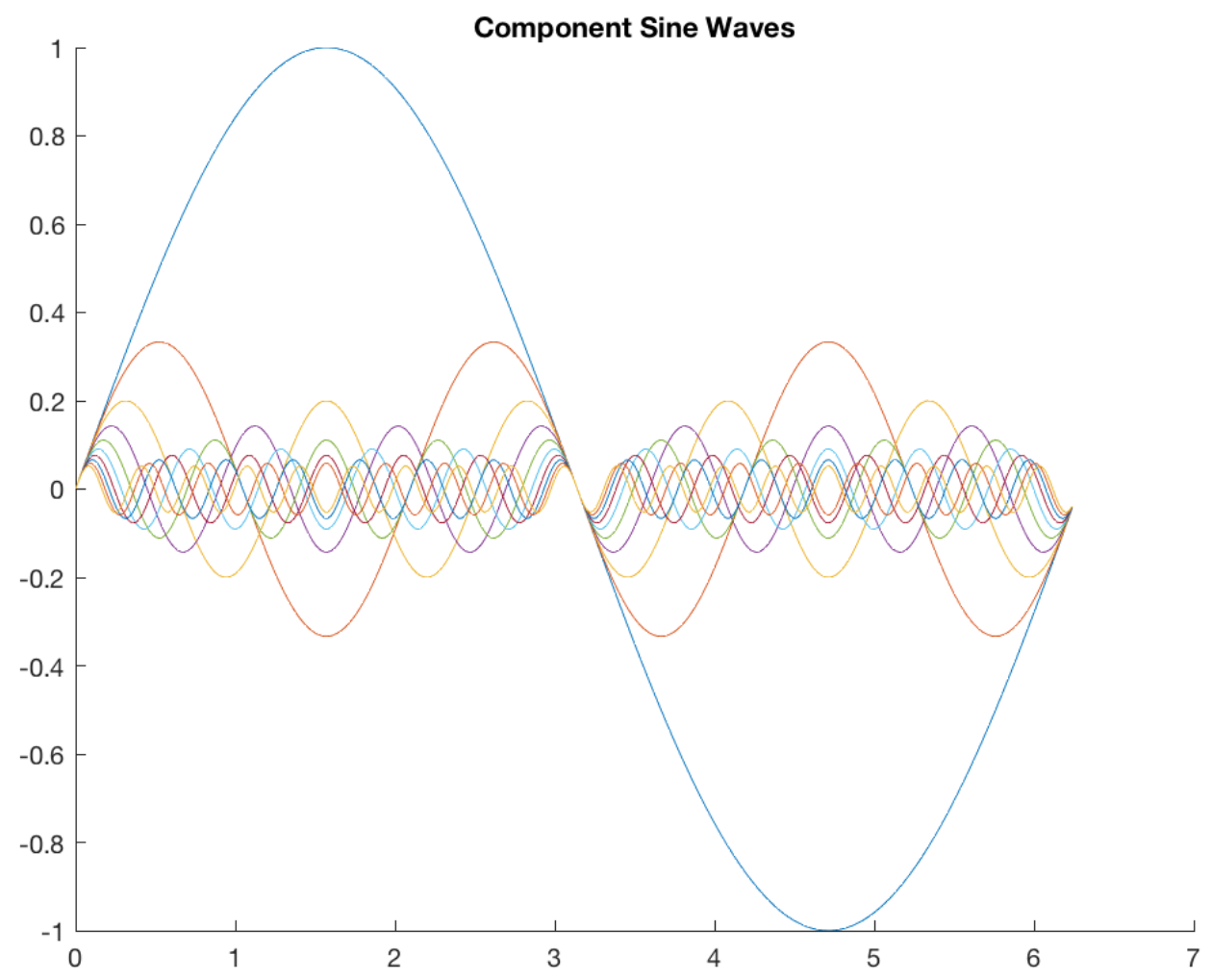

Figure 3.2: The sinusoids making up a square wave.

The transform is shown mathematically below:

$$
X(\omega)=\int_{-\infty}^{\infty} x(t) e^{-j \omega t} d t
$$

This transform is comparing the signal $x(t)$ to a complex exponential where $e^{-j \omega t}=\cos (\omega t)-j \sin (\omega t)$. Using contributions of complex sinusoids allows us to get the magnitude and phase of a component frequency. For every frequency, w, we compare how the signal, $x(t)$, matches at all time points with the complex sinusoids at $w$. In this manner we measure the amount of amplitude and phase that a given 
frequency $w$ is contained in the signal, given by $X(w)$.

$$
X(\omega)=\int_{-\infty}^{\infty} x(t)(\cos (\omega t)-j \sin (\omega t)) d t
$$

The result, $X(w)$, is a measure which shows us the amount and angle of any component frequencies which make up a signal. When we take the Fourier transform of a time domain signal we create a frequency representation of that signal and we are said to be in the frequency domain.

Sometimes a signal changes with time, such as is the case with voice signals. These signals are termed nonstationary. A stationary signal is one in which the statistical parameters of the signal do not change over time. With a nonstationary signal, these statistical descriptions, change in time. We sometimes want to look at how a signal varies in frequency content over time. To do this we window a signal in time and take subsequent Fourier transforms of the windowed signals. We in turn look at the frequency content of a signal as it varies over the different windows. To window a signal means to analyze it in sections. This overall process is called the short time Fourier transform [10]. Mathematically it looks like this:

$$
X(\tau, \omega)=\int_{-\infty}^{\infty} x(t) w(t-\tau) e^{-j \omega t} d t
$$

Notice that the equation is very similar, except now we multiply our signal $x$ by a window $w$ at varying points in time. Our resulting function $X$ depends both on time shift and frequency. Visually we are doing the processing shown in Figure 3.3. 
16
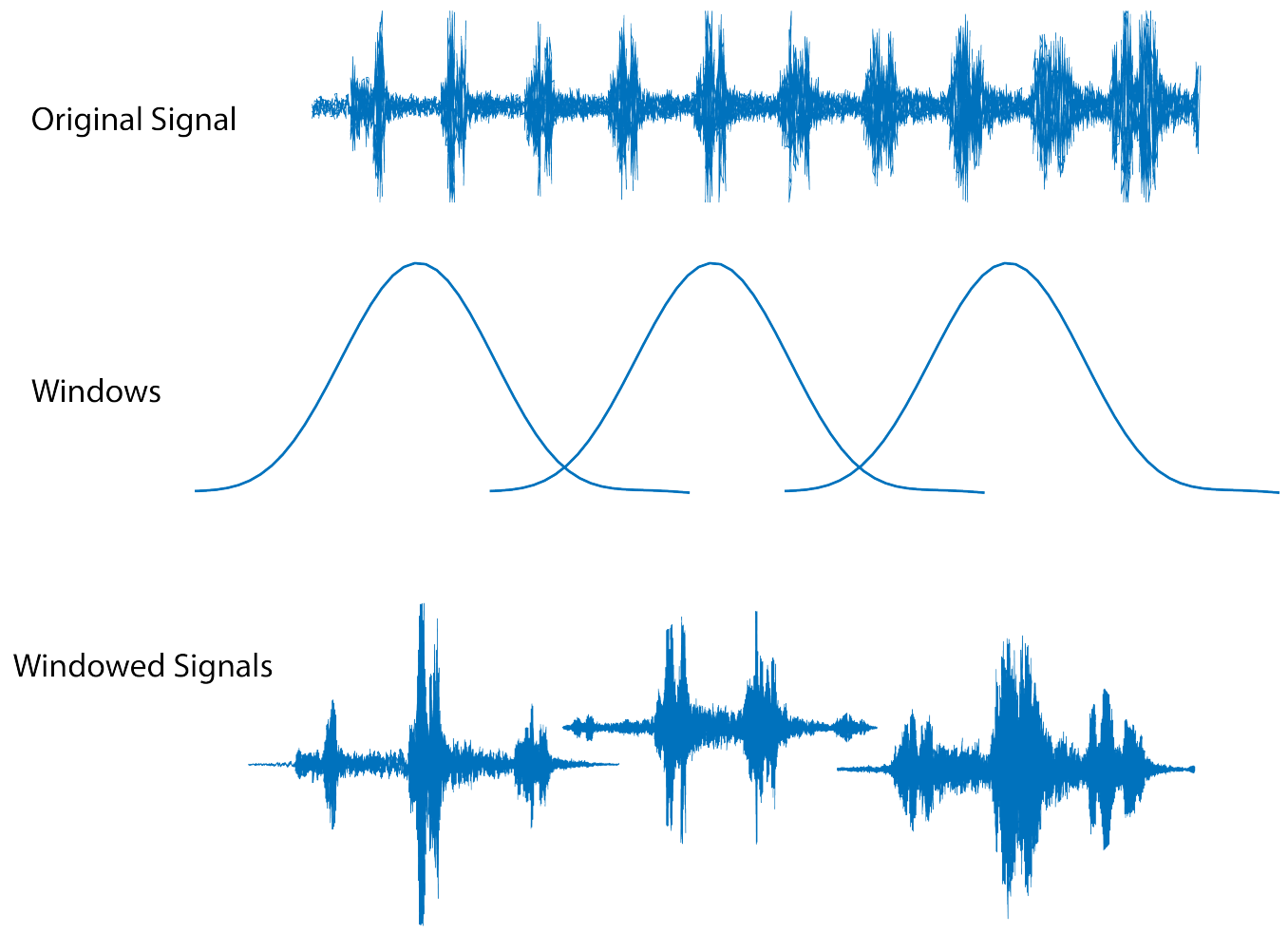

Figure 3.3: Windowing prior to Fourier transform.

We visualize the short time Fourier transform using an energy plot called a spectrogram. The short time Fourier transform gives us a spectrogram when frequency content versus time is plotted. A spectrogram is shown in Figure 3.4. 


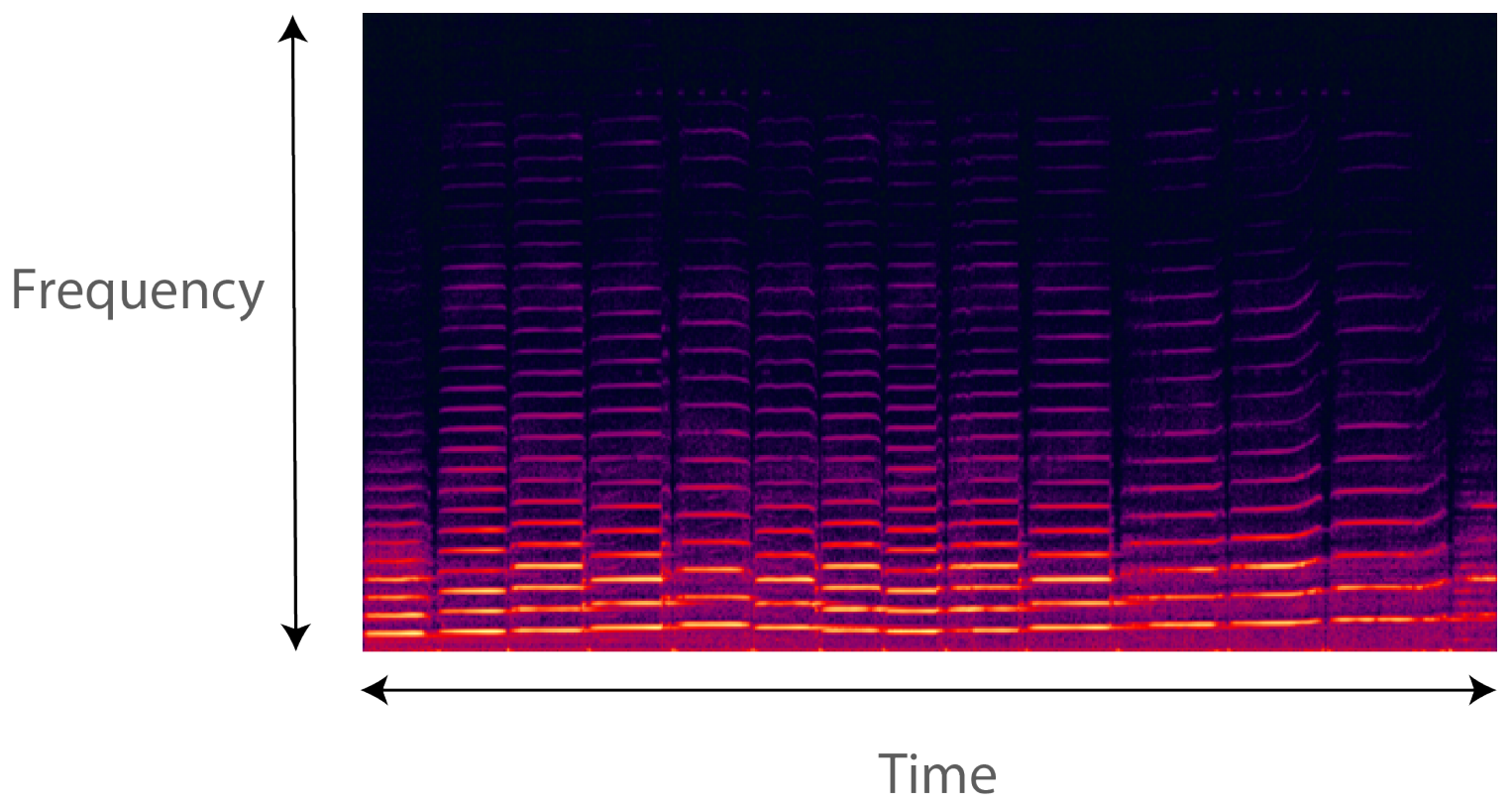

Figure 3.4: Spectrogram.

This spectrogram gives us information about the frequency content of a signal as it varies with time. However, an issue arises with the spectrogram. The spectrogram and in turn the Fourier transform is limited in its ability to resolve a signal which is windowed. The Fourier transform is defined for infinitely long signals [22, 30,31]. When we take a portion of a signal to analyze we inherently apply a window to that signal. This can have a large effect on the spectral analysis. A window applied to a signal is in effect multiplying a signal with a window in time. Resolution of an infinitely long sinusoid is show in Figure 3.5. This perfect resolution is lost when we window. 

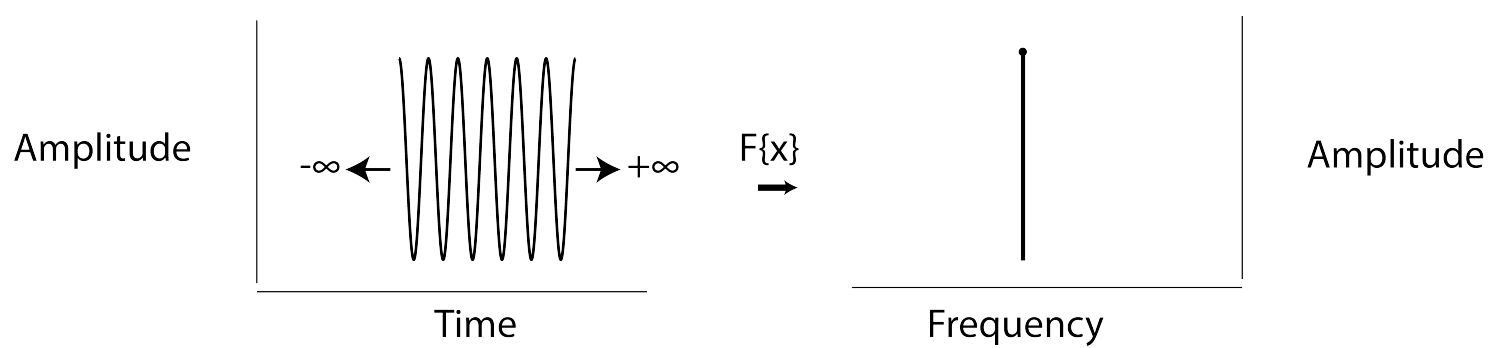

Figure 3.5: Fourier transform of infinite sinusoid.

A central idea of signal processing is the convolution theorem [31]. It states that a multiplication in the time domain is equivalent to the convolution of the two corresponding signals in the frequency domain. Again, we get signals into the frequency domain by taking their Fourier transform. The Fourier transform of an infinite sinusoid of one frequency results in an impulse in the frequency domain. The Fourier transform of a rectangular window is a sinc function. A sinc function is shown in Figure 3.6. As we change the window length in the time domain we also change the sinc's main lobe width in the frequency domain as demonstrated in Figure 3.7. 


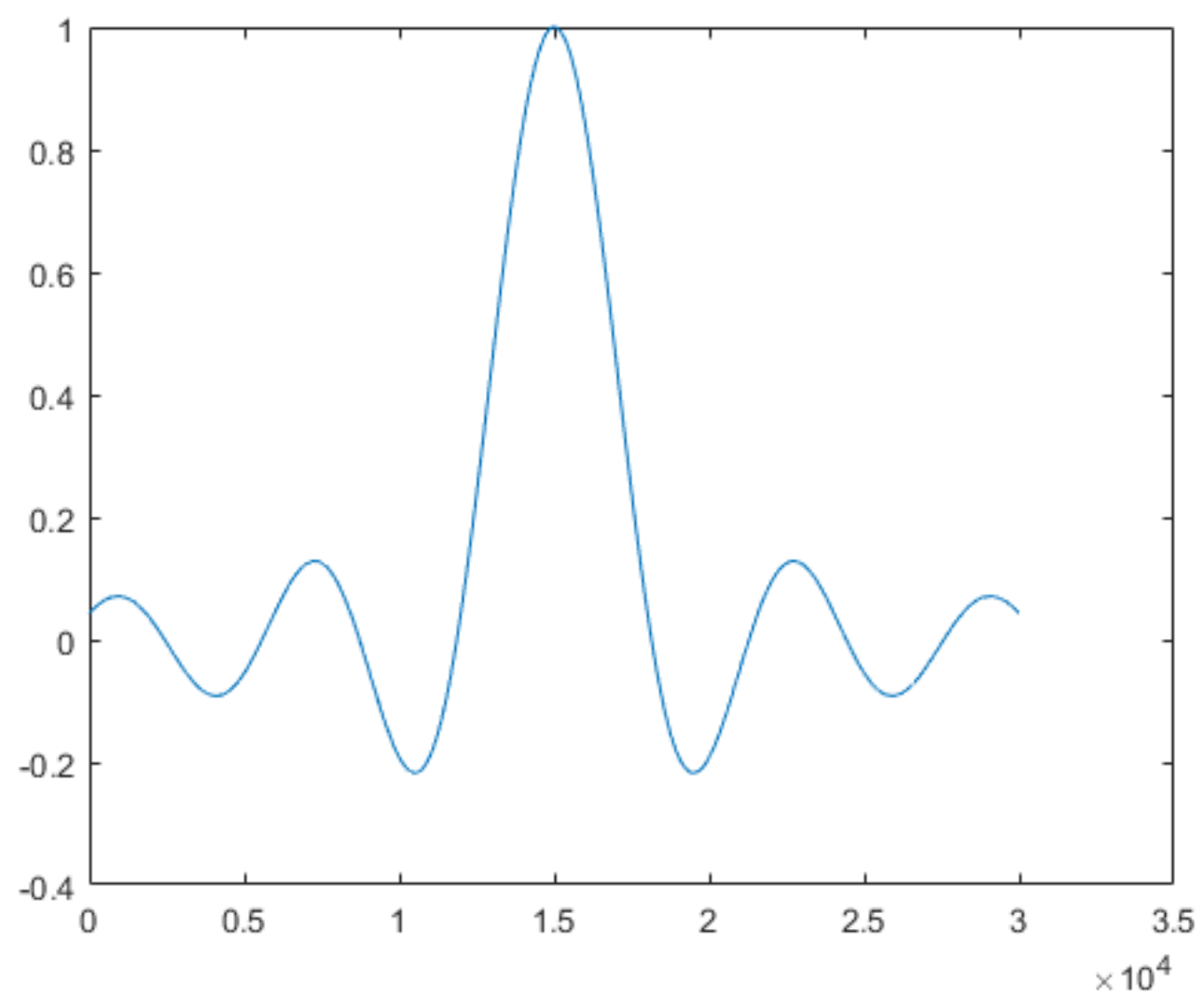

Figure 3.6: Sinc function $\sin (\mathrm{x}) / \mathrm{x}$. 

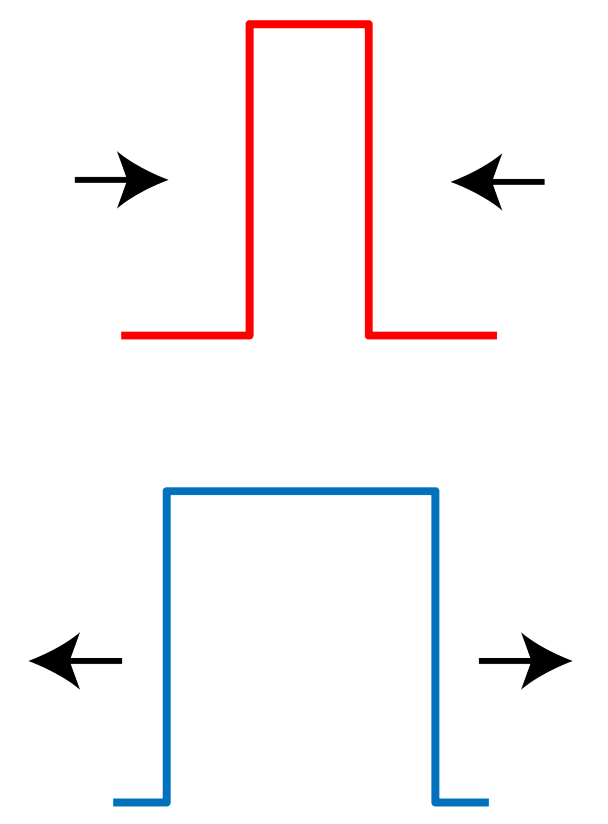

\section{Time}

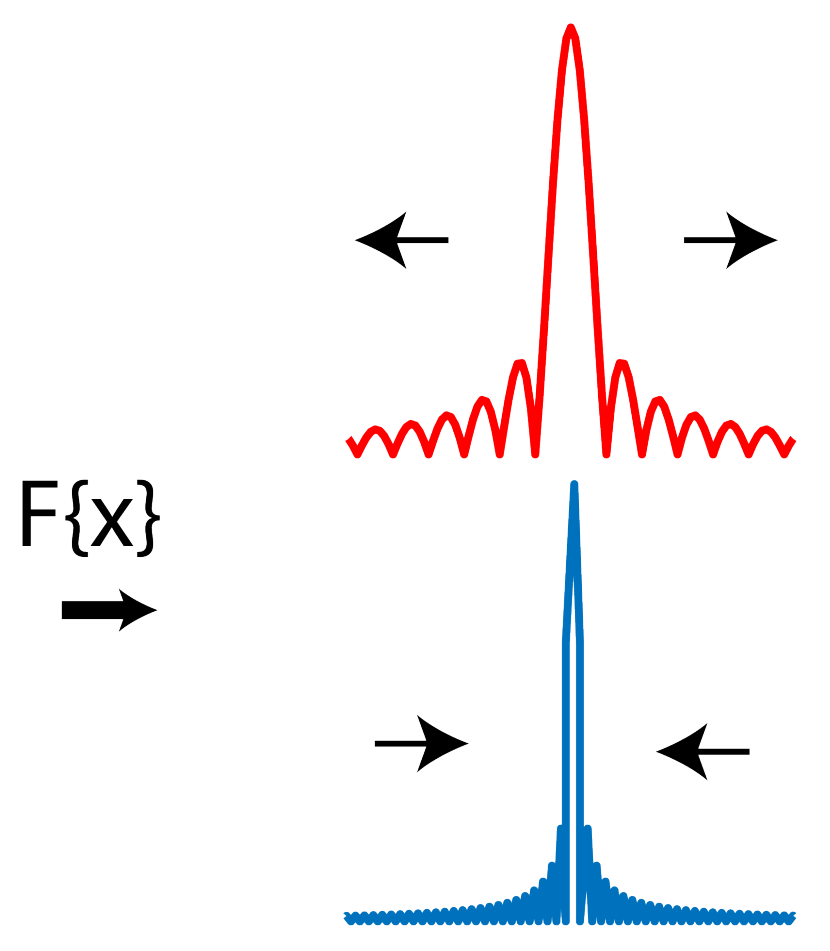

Frequency

Figure 3.7: Varying window lengths and their Fourier transforms.

The convolution theorem of signal processing states that we convolve the two signals in the frequency domain because we multiplied them in the time domain. When we look at only a portion of our sinusoid, we have in effect windowed the signal, multiplying the signal by the window. The two signals are the original infinite sinusoid and the window. Convolution can be thought of as a sliding multiply and add operation or as a sliding dot product. Two signals are slid past each other, multiplied and added for each time delay of one of the signals. The perfect resolution of an infinite sinusoid is now smeared spectrally by a convolution with the window's 
equivalent sinc function in the frequency domain. This results in a lowered resolution of frequency of our signal in the frequency domain. In essence our signal in the frequency domain has been blurred. The convolution theorem is stated below.

$$
F(f \cdot g)=F(f) * F(g)
$$

We now have the multiplication of a window and an infinite sinusoid in the time domain, which results in the convolution of their frequency transforms. The perfect impulse is now convolved with the transform window. The end result has energy spread in the frequency domain, shown right of the arrow in Figure 3.8.

\section{Amplitude}
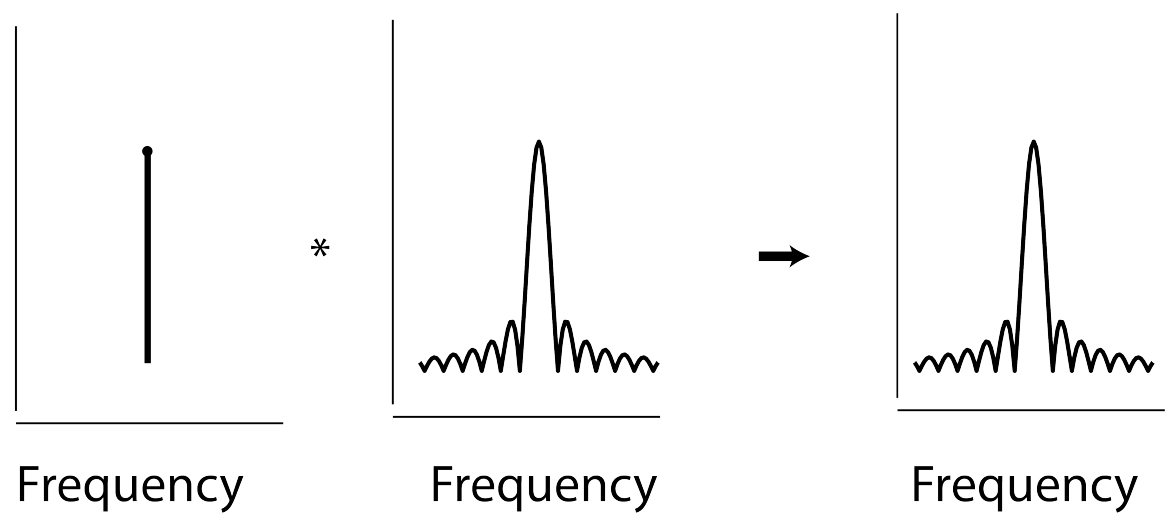

Figure 3.8: Spectral smearing.

The central idea is that a long window in time has a more concentrated main lobe in the frequency domain. When we convolve this with our frequency domain sinusoid, we get a more concentrated spectrum. This more concentrated spectrum allows a higher resolution of frequency. If our signal had two component sinusoids, this smaller main lobe convolved with each sinusoid's transformed impulse would allow better resolution of each component frequency. Thus, when we use a longer window 
we get a better frequency separation and resolution. However, this long window in time, allows more events to happen inside this window. Thus, we cannot separate the events of the window accurately in time. This begins to illustrate the trade-off of frequency and time resolution when we use the short time Fourier transform and in turn the Fourier transform with windows.

This now leads us into the issue of selecting window sizes when using the short time Fourier transform. The short time Fourier transform inherently uses a window to segment the signal under analysis. As described above, this window choice will affect our ability to resolve frequency components of a signal.

The Short Time Fourier Transform

To analyze a signal that changes with time (a non-stationary signal), a tool which is often used is the short time Fourier transform and in turn the spectrogram. The short time Fourier transform windows a signal in time and then takes a Fourier transform of the windowed signal. This idea is presented in Figure 3.9. 

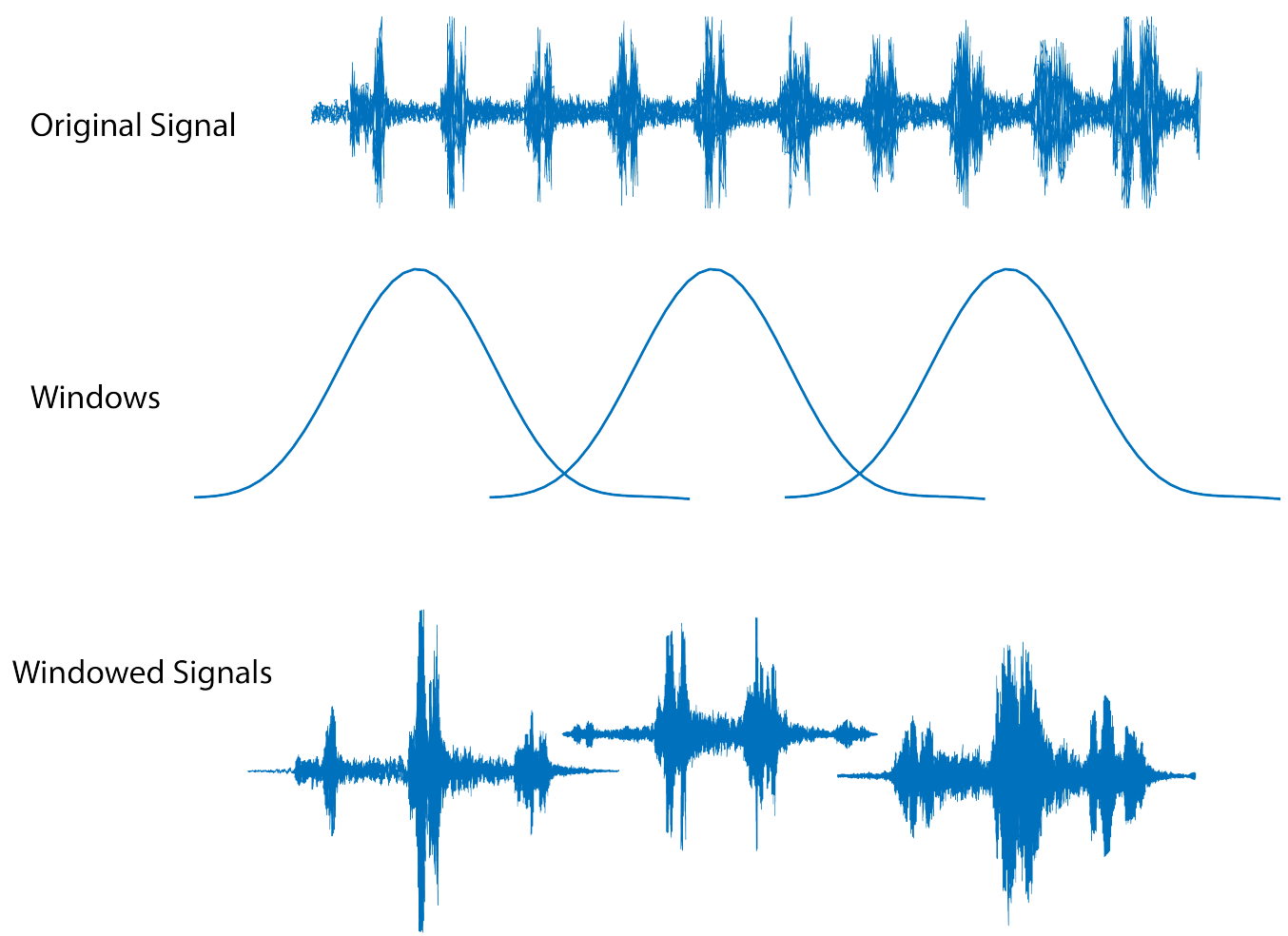

Figure 3.9: Windowing prior to Fourier transform.

We have previously discovered the effect which windowing has on resolution of time events or frequency content. A large window can resolve frequency content well. A short window can resolve time events well. A spectrogram picks one window size across the analysis. This presents a problem. We trade time resolution for frequency resolution. We examine this trade-off in Figure 3.10. 

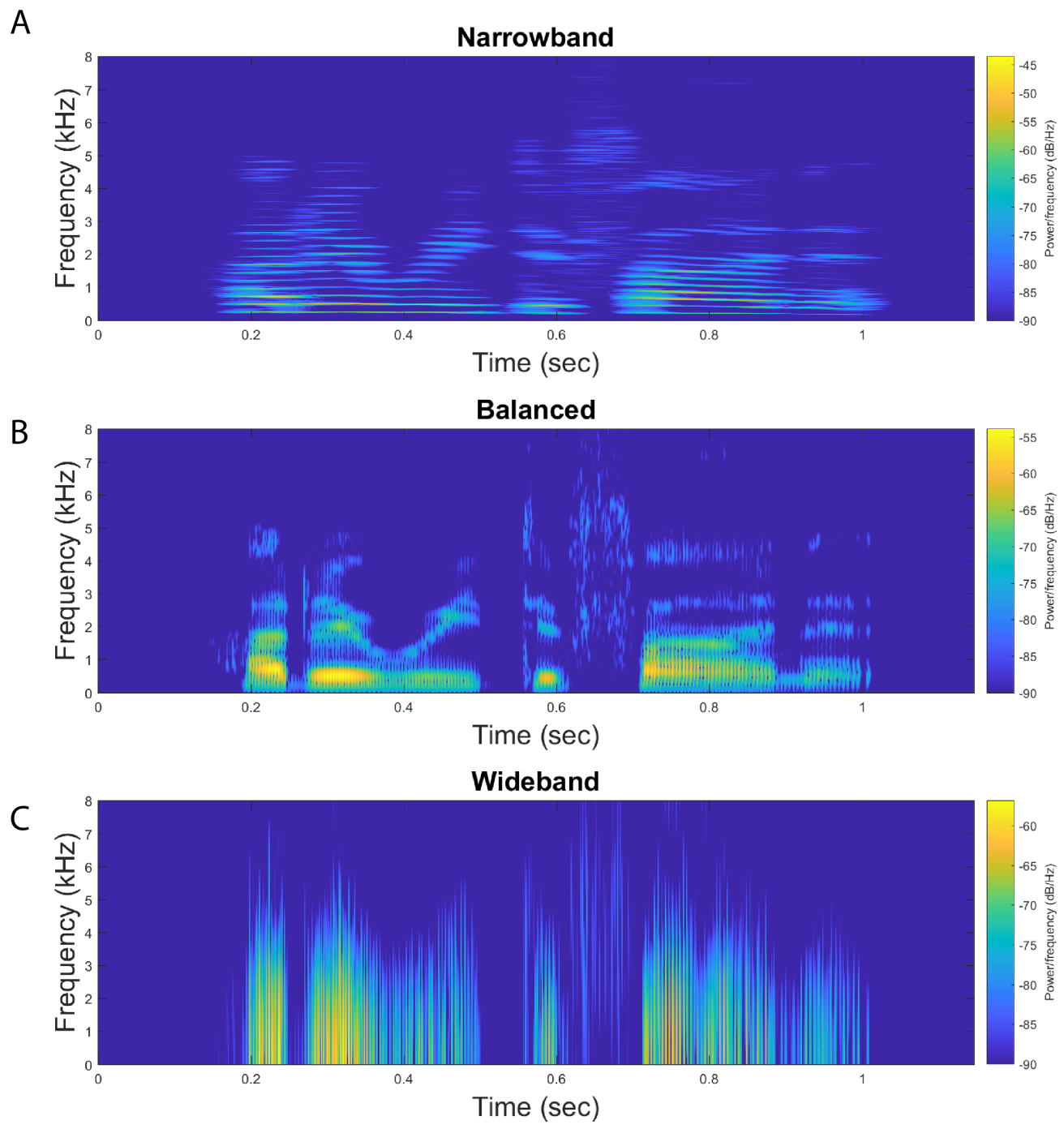

Figure 3.10: Wideband/narrowband spectrogram. The spectrogram window sizes used. Narrowband: .1 Seconds. Balanced: .005 Seconds. Wideband: .0005 Seconds. Evaluated every 25 samples.

In Figure 3.10, we see both a narrowband and a wideband spectrogram. A narrowband spectrogram uses a long window in time for each Fourier transform. This resolves frequency accurately. This is shown in Figure 3.10 A. A balanced spectrogram can also be used. It is a balance between short and long windows so 
frequency transitions can be seen. This is shown in Figure 3.10 B. A wideband spectrogram uses a short window which in turn resolves time events more accurately. This is shown in Figure 3.10 C. In the narrowband spectrogram we see tight frequency bands which are spread out over time. In the wideband spectrogram we can see more evolution of the signal in time, but we also see energy spread over wider ranges of frequencies.

Time-frequency analysis is the study of the time-frequency plane and how to resolve the signals which occupy it [10]. This field of study gives us tools to describe different methods of signal analysis as well as the ability to examine exactly these time-frequency trade-offs. If we look at the time-frequency plane, we can plot the resolving power of different types of short time Fourier transforms. This idea of tiling the time-frequency plane to describe resolving ability is demonstrated in Figure 3.11 and Figure 3.12.

Frequency

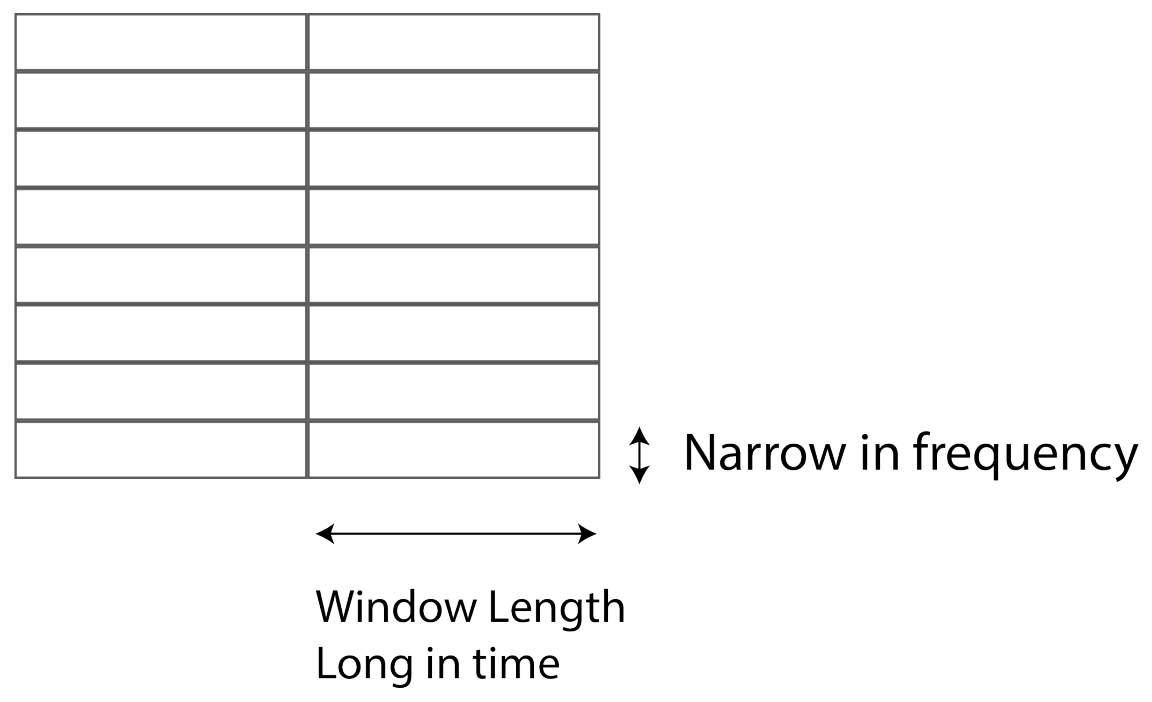

Time

Figure 3.11: Narrowband spectrogram time-frequency tiling. 


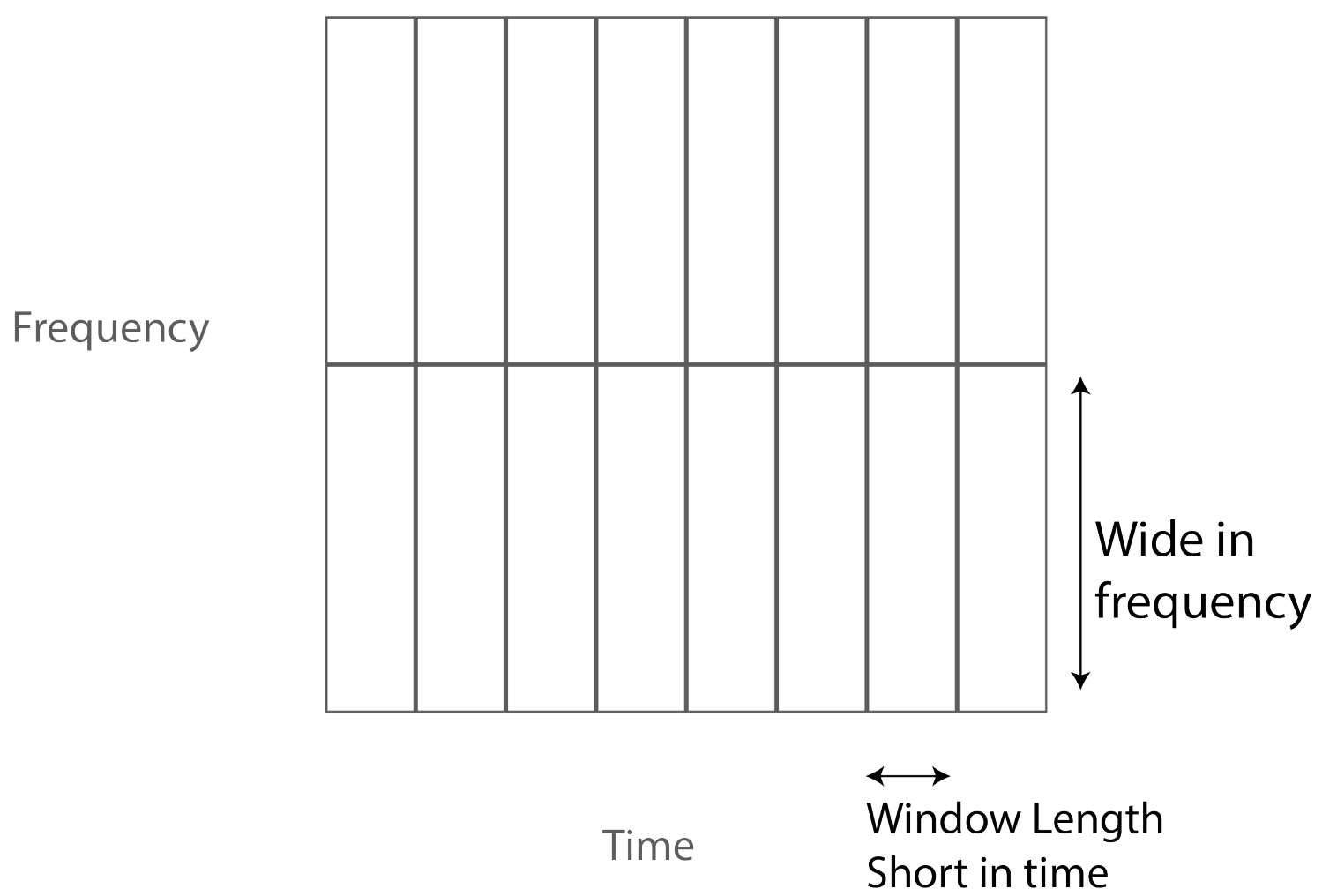

Figure 3.12: Wideband spectrogram time-frequency tiling.

In the narrowband description, we see rectangles which are narrow along the frequency axis indicating high frequency resolution. In the wideband description, we see rectangles which are narrow along the time axis indicating high time resolution. This tiling describes how our analysis tool is resolving signals in either time or frequency. The short time Fourier transform is a common method used to process signals for audio machine learning algorithms $[4,16,17]$. However, users must pick a window size and then proceed to use this window size for their analysis. This discussion now leads to the question of how small can we make these rectangles. To answer this question a central tenet of time-frequency analysis will be used. 
The Uncertainty Principle

Time-frequency analysis has a central theory called the uncertainty principle [17]. It states that a signal's duration and bandwidth cannot both be arbitrarily small. As an example, let us examine one exact moment in time. If we pick this exact point in time, an infinite number of frequencies can travel through this single point in time. We are showing that an infinitesimally small time slice has an infinite bandwidth. This idea is illustrated in Figure 3.13 . The exact moment in time is indicated by the impulse occurring at the center of the figure. Signals of every frequency can pass through this point.

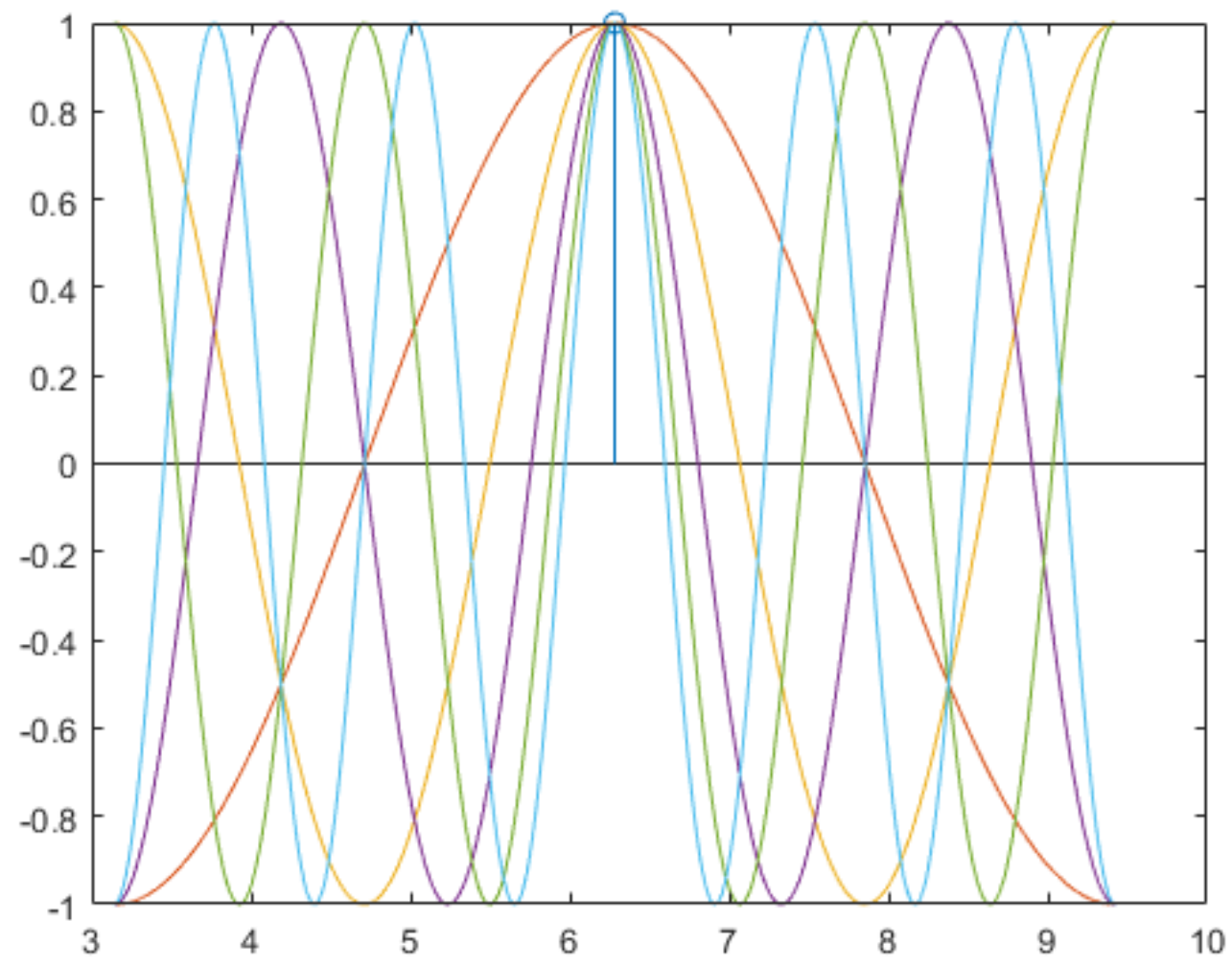

Figure 3.13: Infinite bandwidth through single point in time. 
This idea ties in very closely with the uncertainty principle. Given definitions of the duration and bandwidth of a signal, shown below, an absolute minimum for that signal's duration and bandwidth product can be derived [10]. Duration and bandwidth are defined respectively as variance in time and frequency:

$$
\begin{gathered}
T^{2}=\sigma_{t}^{2}=\int(t-\langle t\rangle)^{2}|s(t)|^{2} d t, \\
B^{2}=\sigma_{w}^{2}=\int(w-\langle w\rangle)^{2}|S(w)|^{2} d t,
\end{gathered}
$$

We can illuminate the idea of mean time, $\langle t\rangle$, and mean frequency, $\langle w\rangle$ used in the above equations. Mean time can be thought of as central time point of a signal. Mean frequency can be thought of as the center of a signal's frequency spread or bandwidth. Duration and bandwidth are defined as variances, $\sigma_{t}^{2}$ and $\sigma_{w}^{2}$. If we take the standard deviation as the square root of the variance, we can further describe the signal. In $2 \sigma_{t} 68 \%$ of the signal will pass in time. $2 \sigma_{w}$ contains most of a signal's frequency contents. This is graphically shown in Figure 3.14 


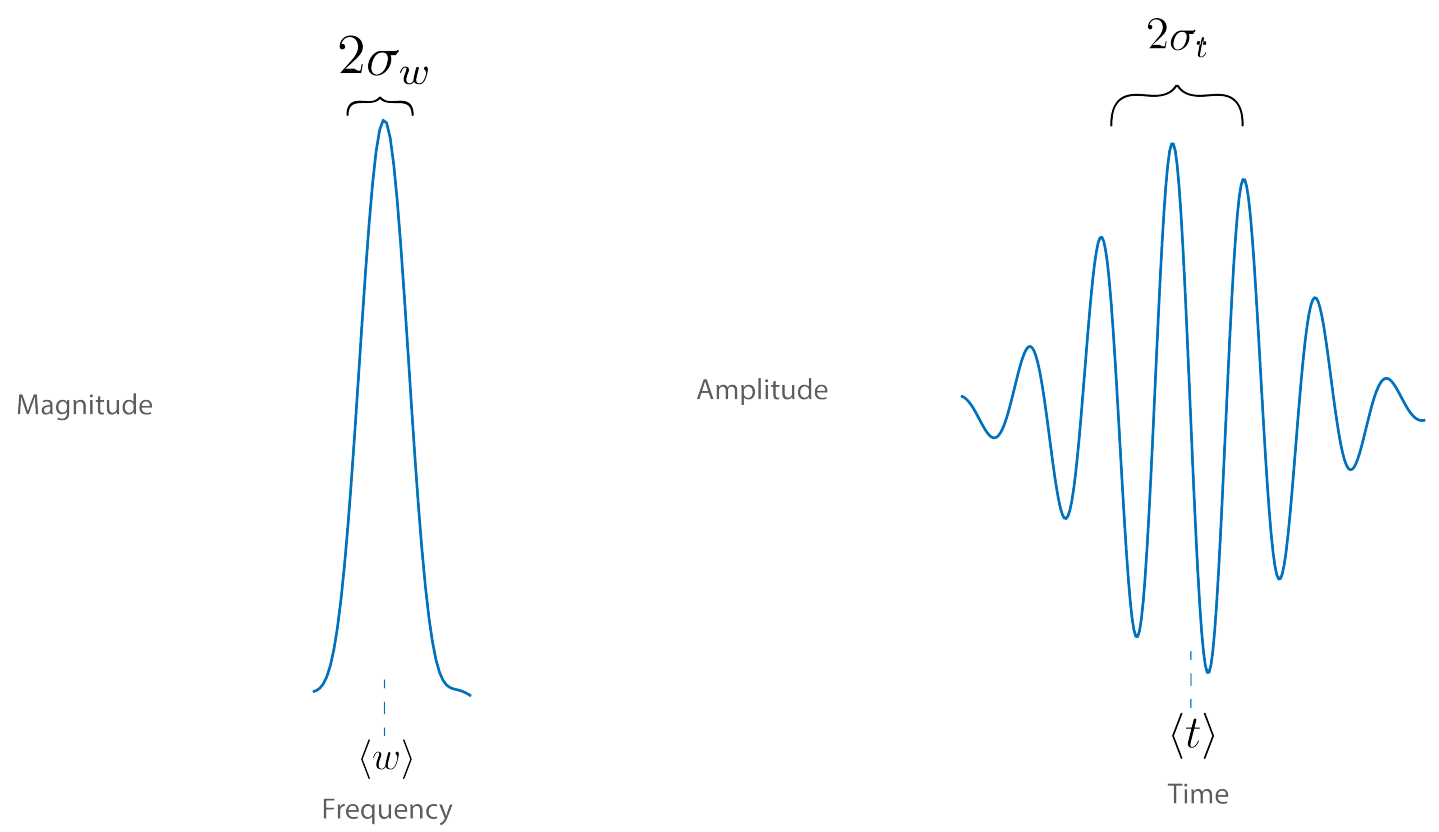

Figure 3.14: Illustration of duration and bandwidth of signal.

The term $|s(t)|^{2}$ represents the energy per unit time at time $\mathrm{t}$ (energy density) [10]. We get the total energy by integrating the signal over time and squaring. The term $|S(w)|^{2}$ represents the energy per unit frequency at frequency w (energy density spectrum) [10]. We get the total energy spectrum by integrating the signal over frequency and squaring.

Given these definitions of duration and bandwidth, a minimum for the product of the two is:

$$
T B \geq \frac{1}{2}
$$

This says that either a signal's bandwidth or its duration can become very small, but not both together.

It can also be shown that the signal which achieves the minimum duration bandwidth product is the Gaussian modulated sinusoid [10]. This signal is shown 
in Figure 3.15. It's area on the time-frequency plane is minimum. A sinusoid is modulated with a Gaussian envelope to make this signal.

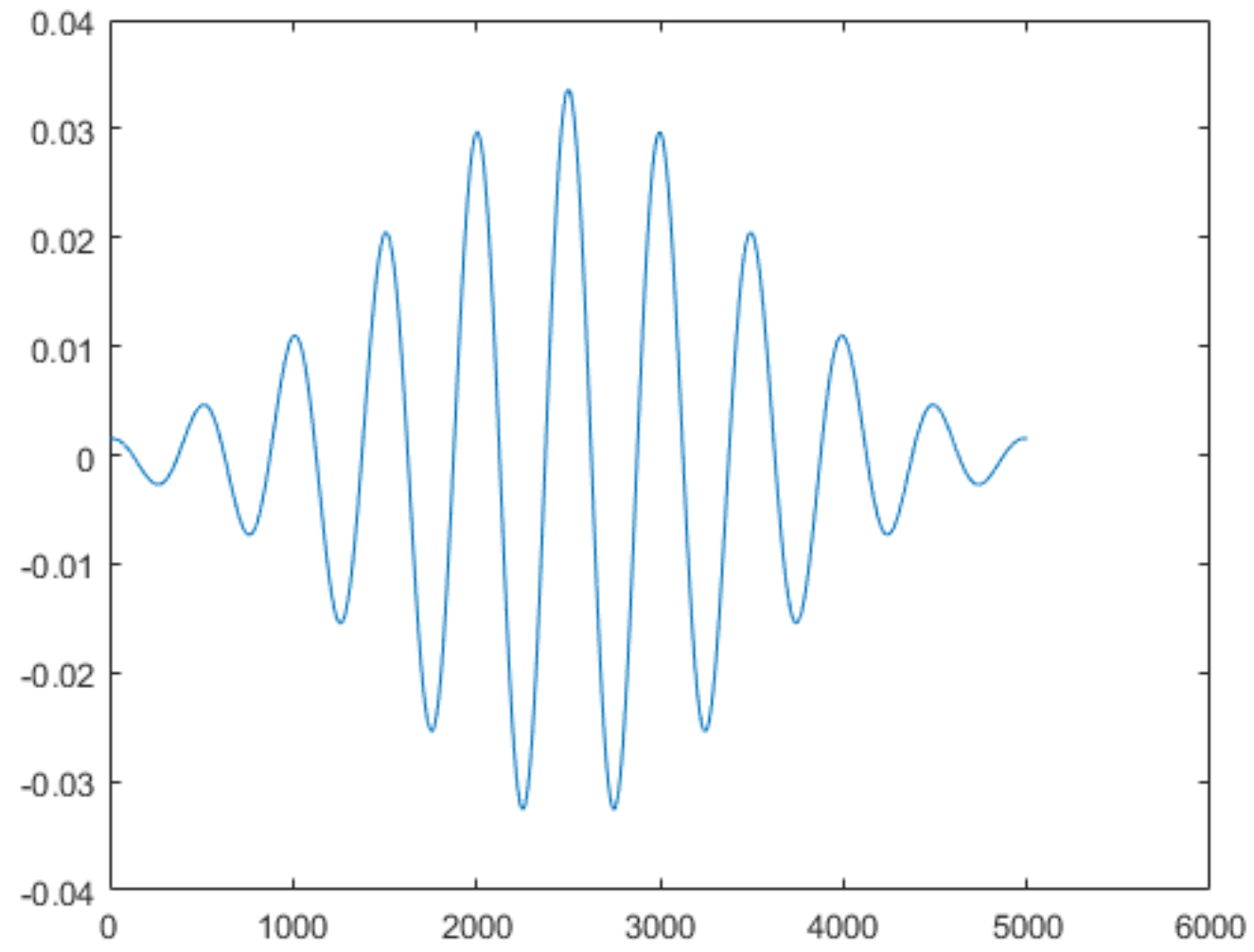

Figure 3.15: Gaussian modulated sinusoid.

This signal achieves the minimum time-frequency product of one half. The mathematical form of the signal is shown below:

$$
s(t)=\sqrt[4]{\frac{\alpha}{\pi}} e^{-\frac{\alpha t^{2}}{2}+j t \omega}
$$

If we can approach the minimum time-frequency resolution with this signal, we now have a tool to tile the time-frequency plane very accurately. This would allow us to identify minimal time-frequency events if we use this signal to match against our 
target signal.

\section{$\mathrm{AM}$ and FM Contributions to Bandwidth}

It can be shown that the bandwith of a signal is the sum of two contributions, that of the AM contribution and the FM contribution to frequency bandwidth [10]. We can find the contribution of each type for a given signal. Large and fast amplitude fluctuations in a signal will contribute to AM bandwidth. Large frequency changes in a signal will contribute to the FM portion of the overall bandwidth. The contributions can be defined mathematically. The same bandwidth as defined previously can now be defined in terms of its AM and FM contributions.

Here $\phi^{\prime}(t)$ represents instantaneous frequency which is the result of the derivative of the phase argument. $A(t)$ represents the amplitude portion of a sinusoid. $A^{\prime}(t)$ represents the derivative of this amplitude portion.

$$
\begin{gathered}
B_{A M}^{2}=\int A^{\prime 2}(t) d t \\
\left.B_{F M}^{2}=\int\left(\phi^{\prime}(t)-\langle w\rangle\right)^{2} A^{2}(t)\right) d t \\
\phi^{\prime}(t)=\frac{d}{d t}(\text { phase }) \\
\langle w\rangle=\int \phi^{\prime}(t) A^{2}(t) d t \\
B^{2}=B_{A M}^{2}+B_{F M}^{2}
\end{gathered}
$$


Thus far we have examined what a spectrogram is and how windowing can affect time or frequency resolution. A spectrogram analysis is chosen with a fixed window size, effectively locking in resolution of time vs frequency. We have also examined the uncertainty principle and the signal which can achieve the minimal area on the time-frequency plane, the Gaussian modulated sinusoid. We also looked at the mathematical definition of the uncertainty principle as well as how to define bandwidth in terms of AM and FM contributions. Now we will look at a Marmoset vocalization signal which contains fine time information, frequency information and time-frequency events which are rotated along the time-frequency axis. This signal type can inform a new tiling of the time-frequency plane, which will become the audio front end of this project. 


\section{CUSTOM TILING OF THE TIME-FREQUENCY PLANE}

We will look at a signal where we need to create a custom tiling and where the standard spectrogram doesn't represent the signal well. This signal is the Marmoset twitter call. The project has chosen the Marmoset vocal repertoire as a basis for examination as well as a test method for the artificial auditory fovea being described. The reason for this is primarily that the Marmoset is a primate animal model used extensively for auditory neurology research $[18,27]$. The Marmoset has a well studied set of calls used for its socialization [3]. These vocalizations are important for those studying Marmoset behavior and auditory neurology. For these reasons, the Marmoset vocalization set was chosen for testing. Figure 4.1 below shows a balanced spectrogram of a Marmoset twitter call. 


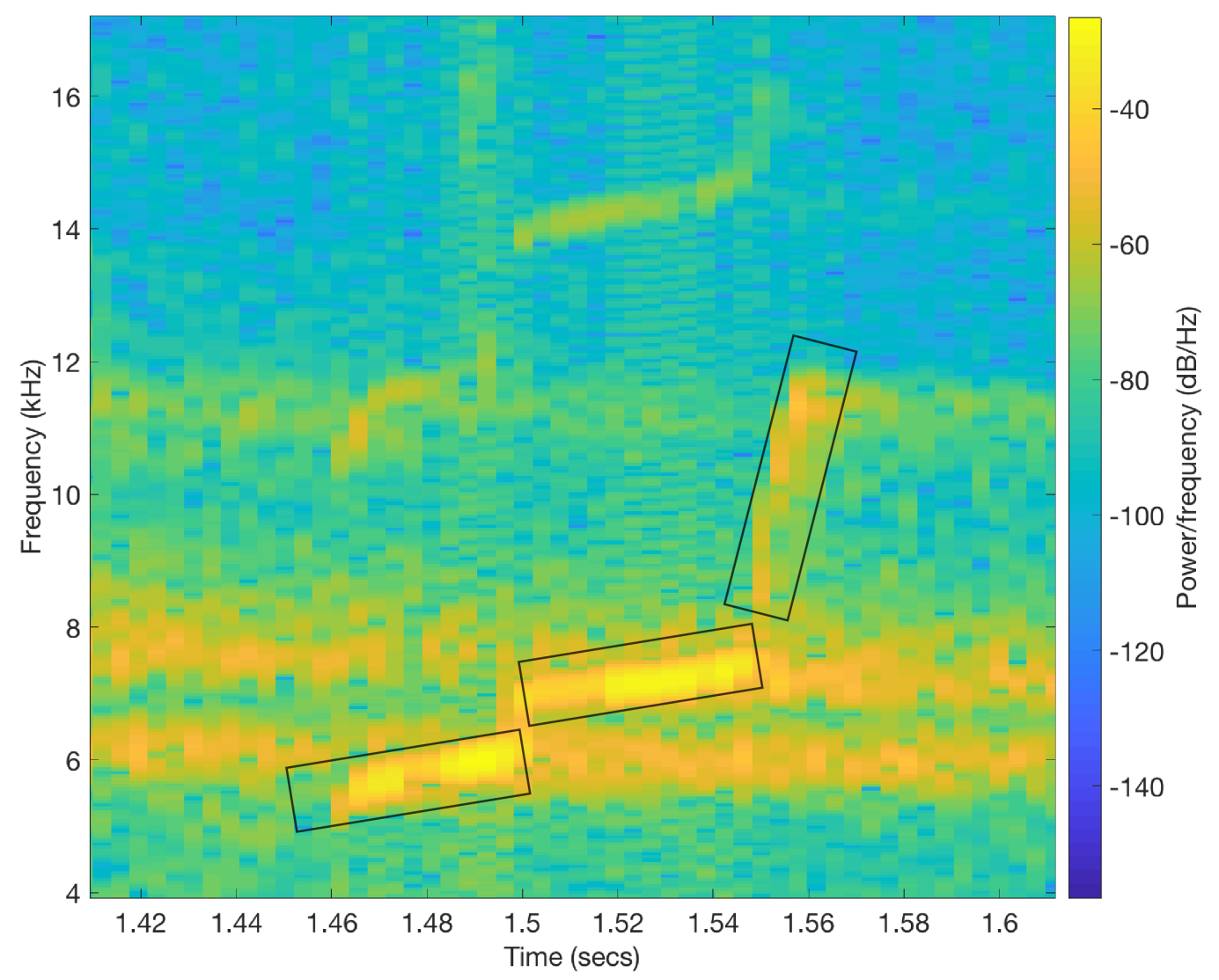

Figure 4.1: Marmoset twitter call and proposed tiles. Balanced spectrogram : .005 second window size.

The spectrogram shows distinct frequency and time events over a portion of a Marmoset call. However, some spectral events are rotated in the time-frequency domain, i.e., there are FM components present. This suggests that a rotated rectangle in the time-frequency domain would be a better match for this portion of the signal. Using a frequency varying sinusoid, we can achieve just this. A variation on the Gaussian modulated sinusoid can help us achieve the rotations in the time-frequency domain. This signal has been presented in literature and is termed the chirplet [23,24]. The chirplet is simply a Guassian modulated sinusoid where the sinusoid's frequency 
is swept. The varying kinds of chirp signals and their space in the time-frequency plane are shown in Figures 4.2 to 4.4. A chirplet which does not change frequency and does not result in rotation in the time-frequency plane is show in Figure 4.2.
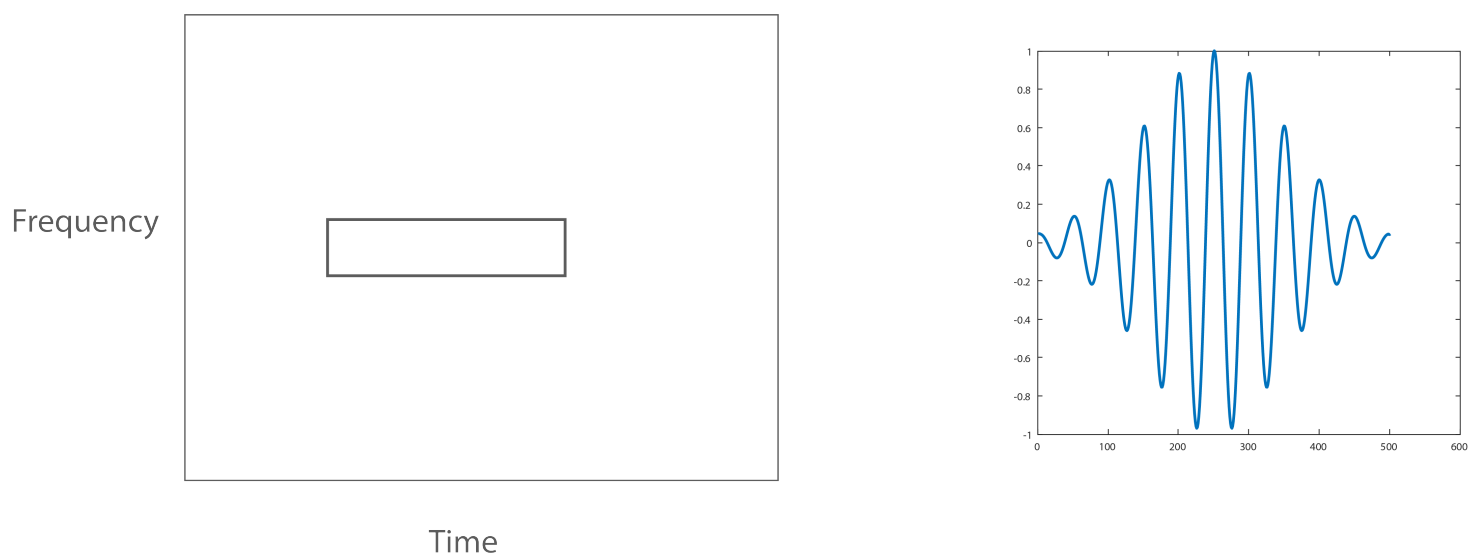

Figure 4.2: Chirp with no FM and tiling. Chirp is centered at $1000 \mathrm{~Hz}$ with a 0 $\mathrm{Hz} / \mathrm{sec}$ slope.

However, if we want to rotate the tiling rectangle, we can. We change the frequency content of the chirplet. We can up sweep and down sweep the frequency of chirplet. Those tilings appear in Figure 4.3 and Figure 4.4.
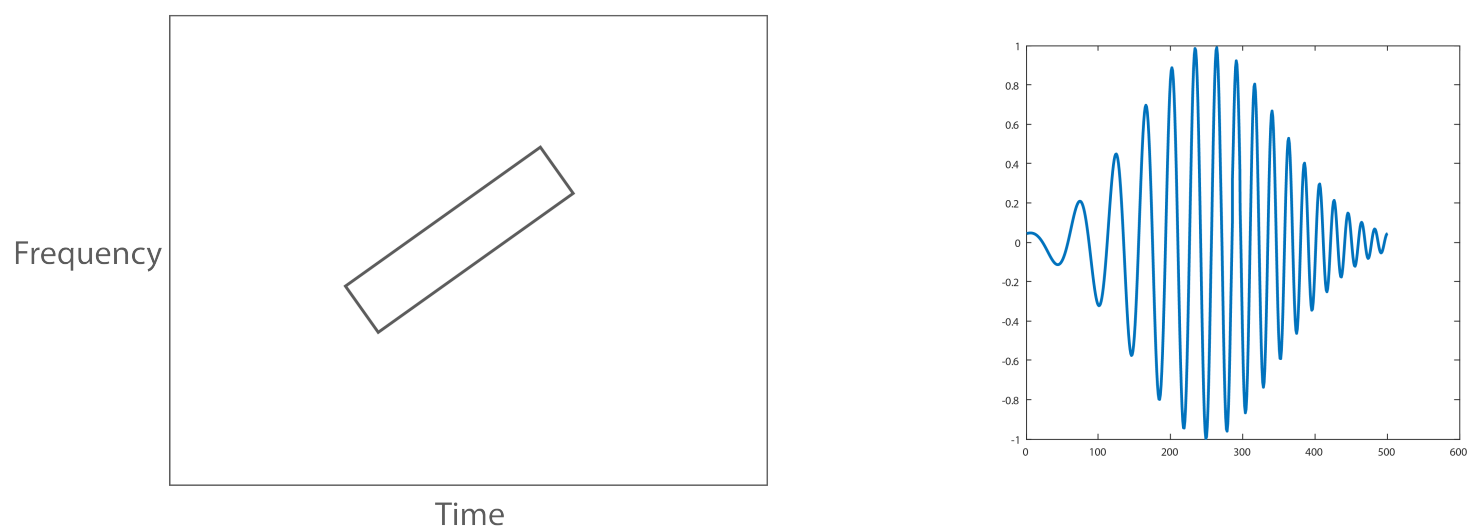

Figure 4.3: Upward chirp. Chirp is centered at $1700 \mathrm{~Hz}$ with a $235 \mathrm{kHz} / \mathrm{sec}$ slope. 

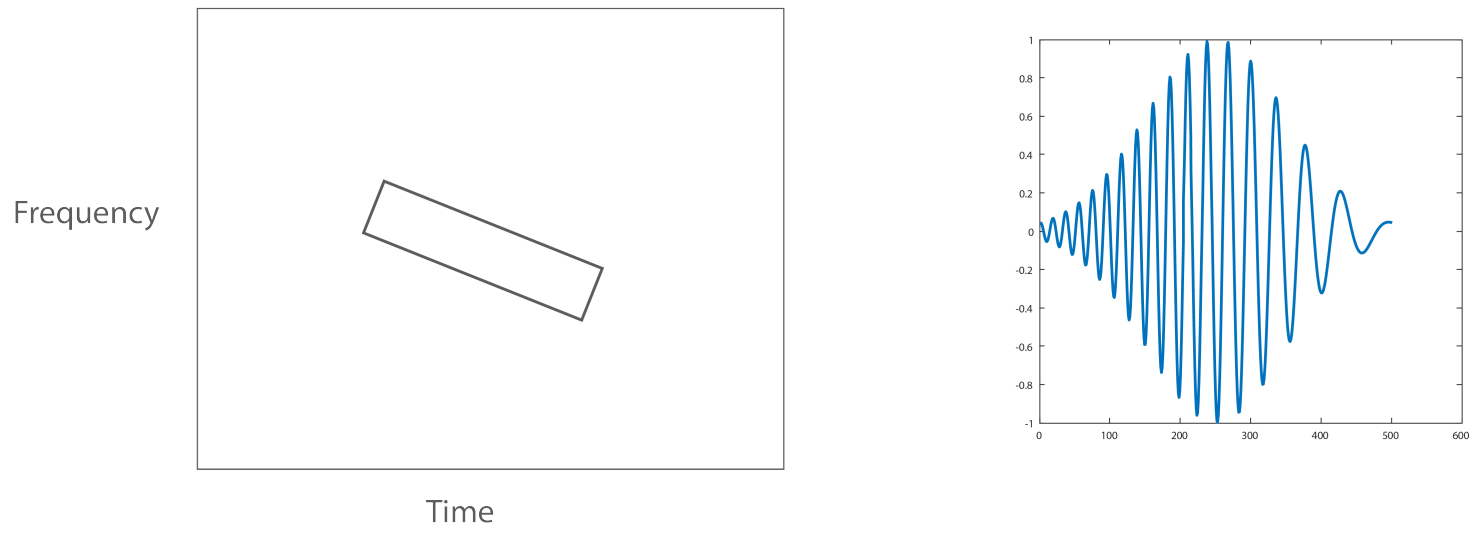

Figure 4.4: Downward chirp. Chirp is centered at $1700 \mathrm{~Hz}$ with a $-235 \mathrm{kHz} / \mathrm{sec}$ slope.

In the case of the upward and downward chirps we can see the frequency of the chirp changing with time. A high to low frequency change is shown in the downward chirp of Figure 4.4 and the opposite for Figure 4.3. The mathematical expression for a Gaussian modulated frequency varying chirp is shown below:

$$
s(t)=\sqrt[4]{\frac{\alpha}{\pi}} e^{-\frac{\alpha t^{2}}{2}+\frac{j \beta t^{2}}{2}+j t \omega_{0}}
$$



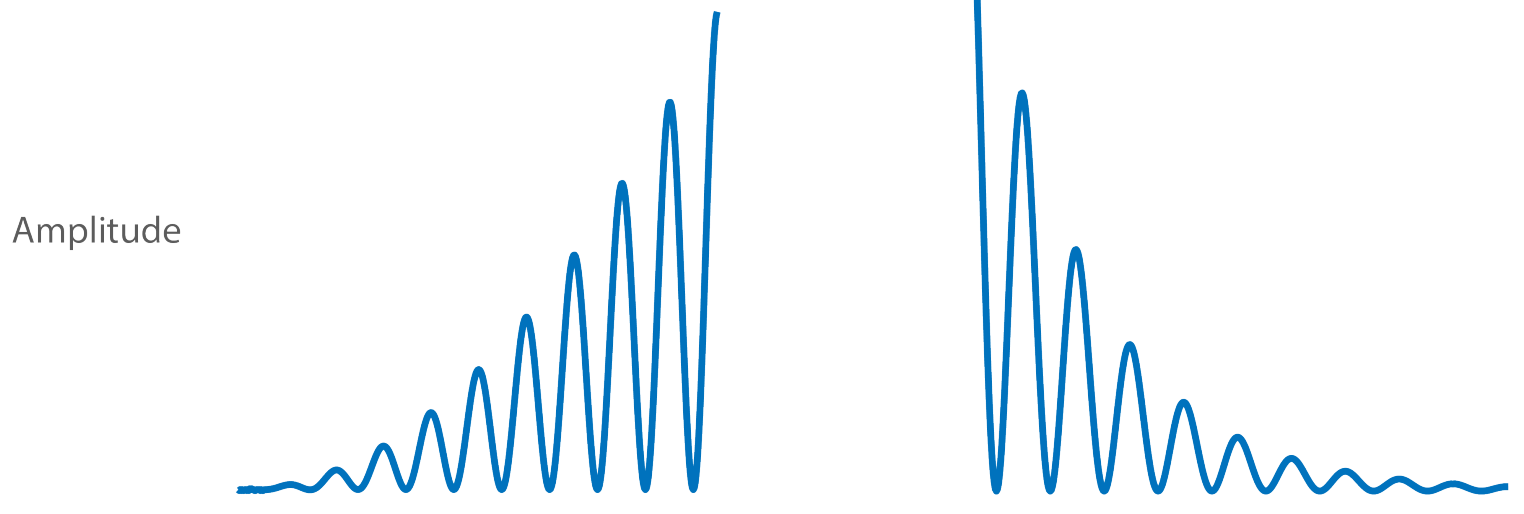

Time

Figure 4.5: Two chirplets under differing envelopes.

We can also look at detecting onsets and offsets by changing the modulation type from a Guassian to a linear ramp. This inspiration comes from neurons which specifically mark offset and onset timings for incoming signals. We use these chirp types later to look at detecting signal onsets and offsets. The six different combinations of onset and offset detectors as visualized on the time-frequency plane are shown in Figure 4.6 to 4.11 .

The associated equation for the onset chirp is:

$$
s(t)=\alpha t e^{\frac{j \beta t^{2}}{2}+j \omega_{0} t}
$$

The associated equation for the offset chirp is:

$$
s(t)=(1-\alpha t) e^{\frac{j \beta t^{2}}{2}+j \omega_{0} t}
$$



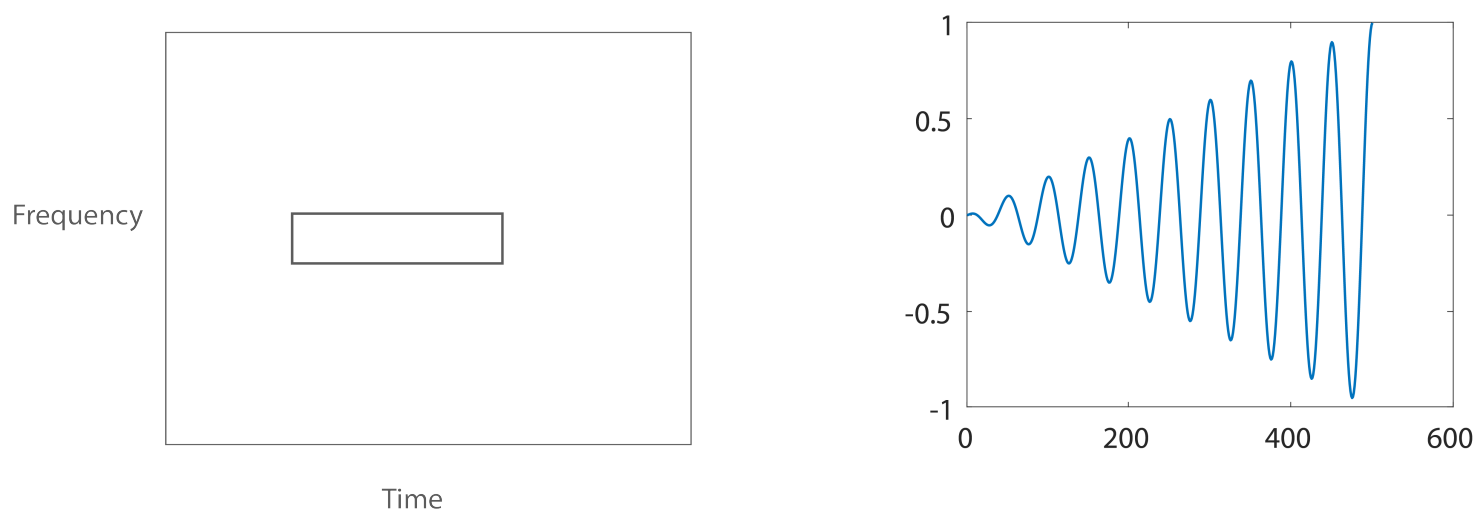

Figure 4.6: Linear chirp and tiling. Onset modulation. Chirp is centered at $1000 \mathrm{~Hz}$ with a $0 \mathrm{~Hz} / \mathrm{sec}$ slope.
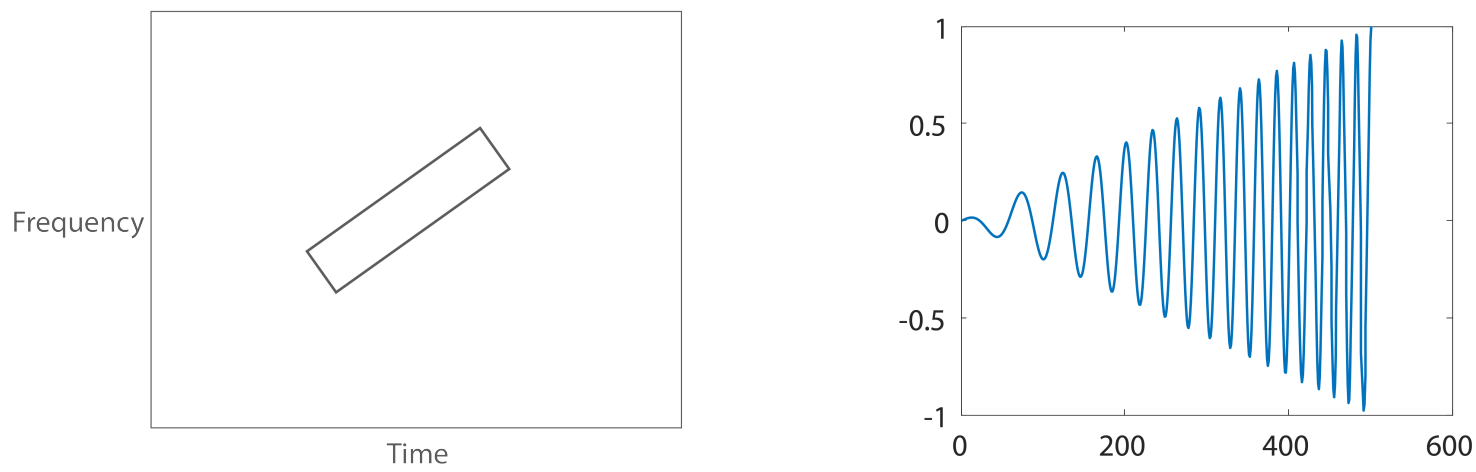

Figure 4.7: Upward chirp. Onset modulation. Chirp is centered at $1700 \mathrm{~Hz}$ with a $235 \mathrm{k} 5 \mathrm{~Hz} / \mathrm{sec}$ slope. 

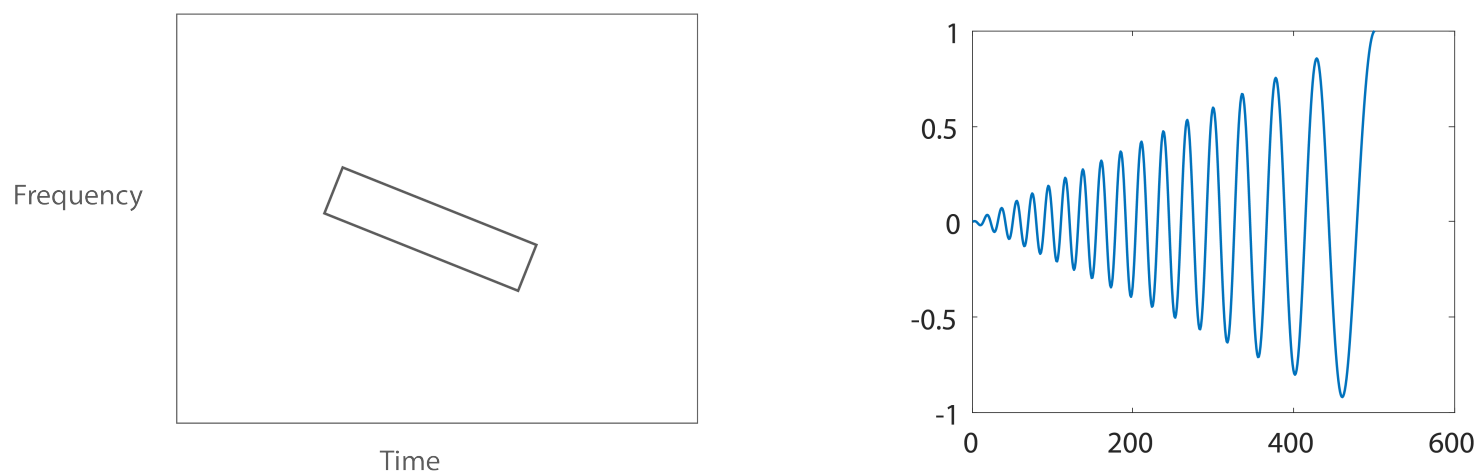

Figure 4.8: Downward chirp. Onset modulation. Chirp is centered at $1700 \mathrm{~Hz}$ with a $-235 \mathrm{kHz} / \mathrm{sec}$ slope.
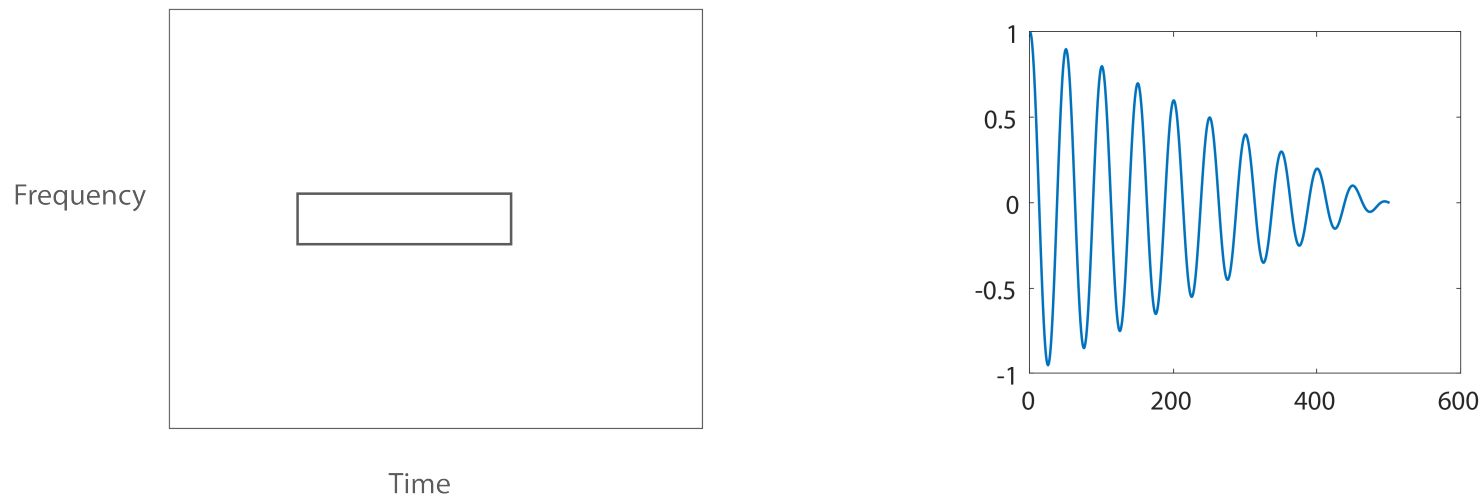

Figure 4.9: Linear chirp and tiling. Offset modulation. Chirp is centered at $1000 \mathrm{~Hz}$ with a $0 \mathrm{~Hz} / \mathrm{sec}$ slope.
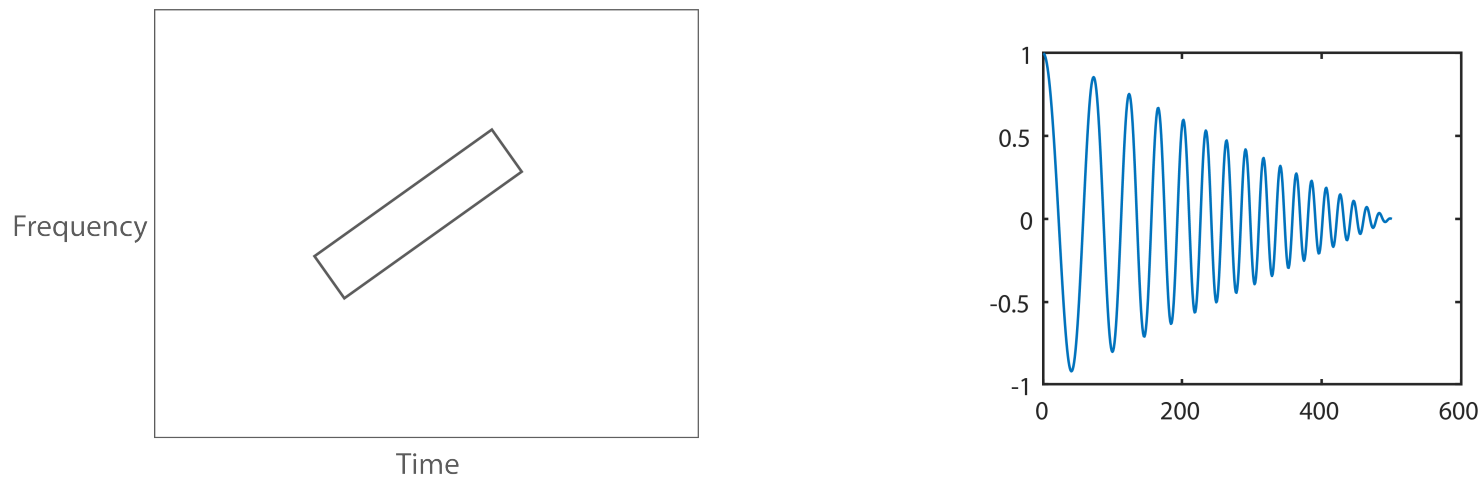

Figure 4.10: Upward chirp. Offset modulation. Chirp is centered at $1700 \mathrm{~Hz}$ with a $235 \mathrm{kHz} / \mathrm{sec}$ slope. 

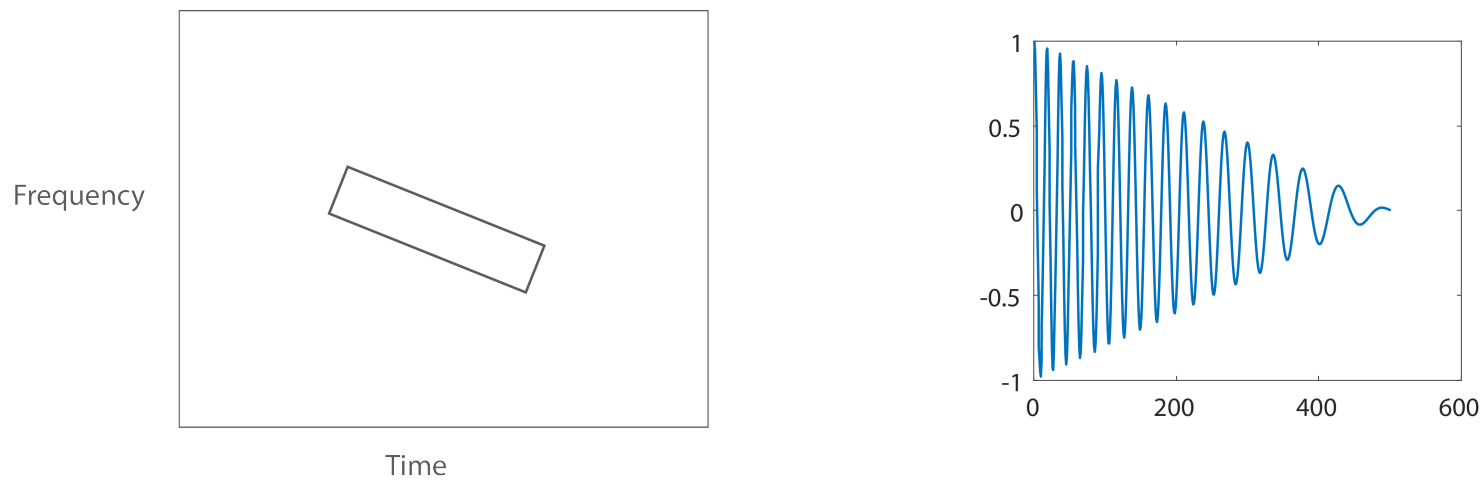

Figure 4.11: Downward chirp. Offset modulation. Chirp is centered at $1700 \mathrm{~Hz}$ with a $-235 \mathrm{kHz} / \mathrm{sec}$ slope.

For mathematical completness, the AM and FM equations will be shown for Guassian modulated and ramp modulated chirps. The total bandwidth of a signal comes from both AM and FM contributions.

We can look at the AM and FM equations for the Guassian modulated chirplet. The mathematical analyses shown here agree up with Cohen's final results for the chirp type waveform [10]. The associated equations for the Guassian modulated chirp follow.

The Guassian modulated chirp is defined as:

$$
s(t)=(\alpha / \pi)^{\frac{1}{4}} e^{-\frac{\alpha t^{2}}{2}+\frac{1}{2} \beta j t^{2}+j t \omega}
$$

Signal envelope is defined as:

$$
A(t)=(\alpha / \pi)^{\frac{1}{4}} e^{-\frac{\alpha t^{2}}{2}}
$$


Phase term is defined as:

$$
\phi(t)=\frac{1}{2} \beta j t^{2}+j t \omega
$$

The AM bandwidth of the signal is defined and evaluated:

$$
\begin{gathered}
B_{A M}^{2}=\int_{-\infty}^{\infty} A^{\prime}(t)^{2} d t=\int_{-\infty}^{\infty}\left(\frac{\partial}{\partial t}\left(\sqrt[4]{\frac{\alpha}{\pi}} e^{-\frac{1}{2} \alpha t^{2}}\right)\right)^{2}=\int_{-\infty}^{\infty} \frac{\alpha^{5 / 2} t^{2} e^{-\alpha t^{2}}}{\sqrt{\pi}} \\
B_{A M}^{2}=\int_{-\infty}^{\infty} \frac{\alpha^{5 / 2} t^{2} e^{-\alpha t^{2}}}{\sqrt{\pi}}=\frac{\alpha}{2}
\end{gathered}
$$

The FM bandwidth of the signal is defined:

$$
B_{F M}^{2}=\int\left(\phi^{\prime}(t)-\langle w\rangle\right)^{2} A(t)^{2} d t
$$

Mean frequency is calculated:

$$
\langle w\rangle=\int_{-\infty}^{\infty} \phi^{\prime}(t) A^{2}(t) d t=\int_{-\infty}^{\infty}(\beta t+\omega) *\left((\alpha / \pi)^{\frac{1}{4}} e^{\left(-\alpha t^{2} / 2\right)}\right)^{2}=\omega
$$

The derivative of the phase term is needed:

$$
\phi^{\prime}(t)=\frac{d}{d t}(\text { phase })=\frac{d}{d t}\left(\frac{\beta t^{2}}{2}+\omega t\right)=\beta t+\omega
$$

The FM bandwidth is evaluated:

$$
B_{F M}^{2}=\int_{-\infty}^{\infty}(\beta t)^{2} *\left((\alpha / \pi)^{\frac{1}{4}} e^{\left(-\alpha t^{2} / 2\right)}\right)^{2}=\frac{\beta^{2}}{2 \alpha}
$$


We see that for a Guassian modulated chirplet, the AM contribution to bandwidth is tied to the $\alpha$ parameter. This $\alpha$ parameter controls the lobe width of the guassian modulation. The FM contribution is tied to both the $\alpha$ and $\beta$ parameters, but more strongly to $\beta$. The $\beta$ parameter tells us the chirp slope on the time frequency plane. A larger $\beta$ gives us a chirp which changes in frequency faster. This mathematical analysis expands and verifies Cohen's AM FM analysis of the Guassian chirp [10]. A demo of the AM FM trade-off for the Guassian modulated chirplet is given in Figure 4.12. 
AM/FM Bandwidth for parameters $\alpha$ and $\beta$
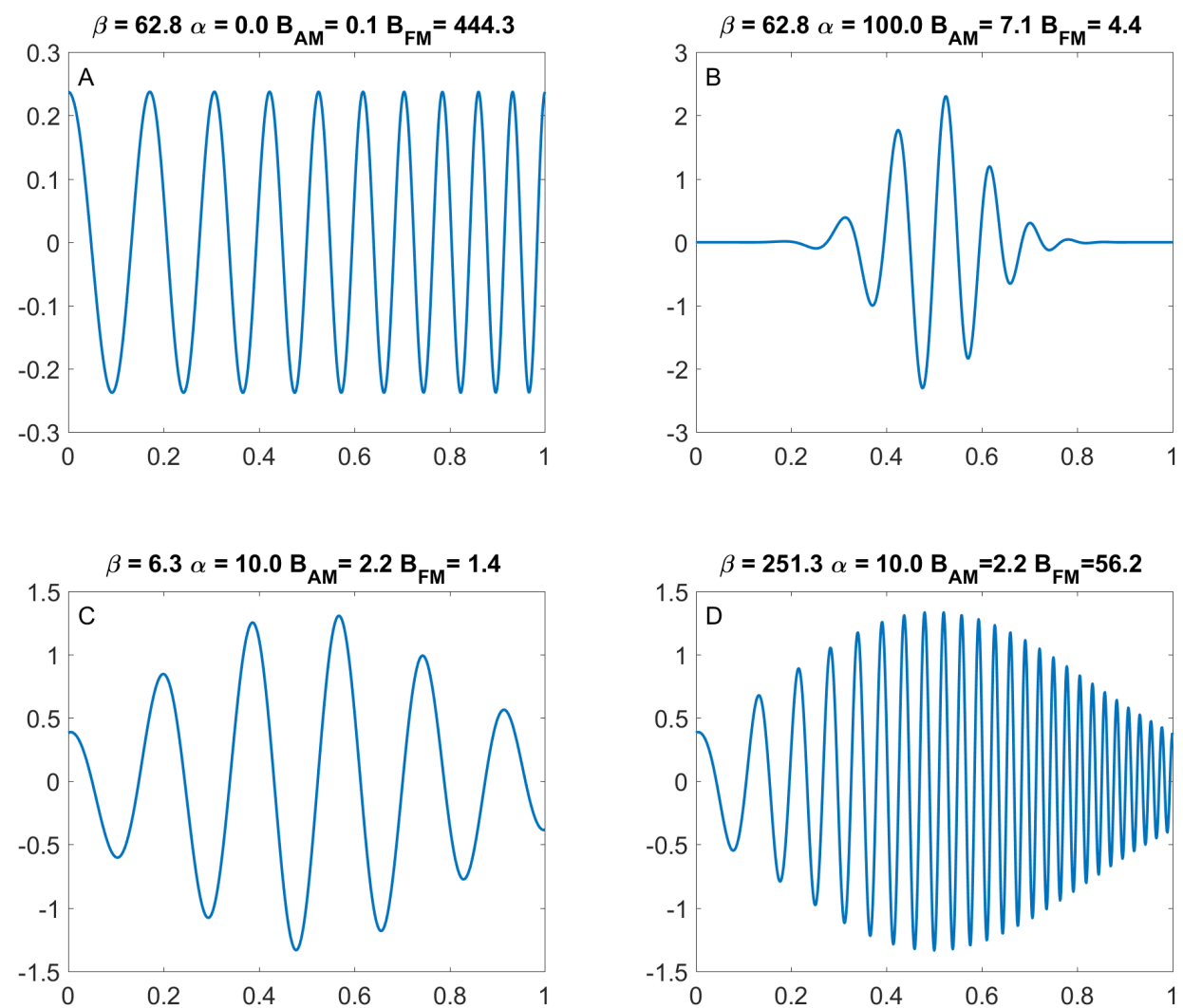

Figure 4.12: A comparison of alternating contributions from either amplitude modulation or frequency modulation. In $\mathrm{A}$, a low alpha parameter compared to $\mathrm{B}$ creates a lower $\mathrm{AM}$ contribution. In $\mathrm{C}$ a lower Beta parameter compared to D creates a lower FM contribution to bandwidth.

A signal with the same bandwidth can be acheived given proportional contributions from either part. Four different configurations of a signal are given in Figure 4.13, where each has a different FM or AM contribution, but all signals have an identical total bandwidth of $4 \mathrm{~Hz}[10,14]$. 
AM/FM Relative Contribution for parameters $\alpha$ and $\beta$
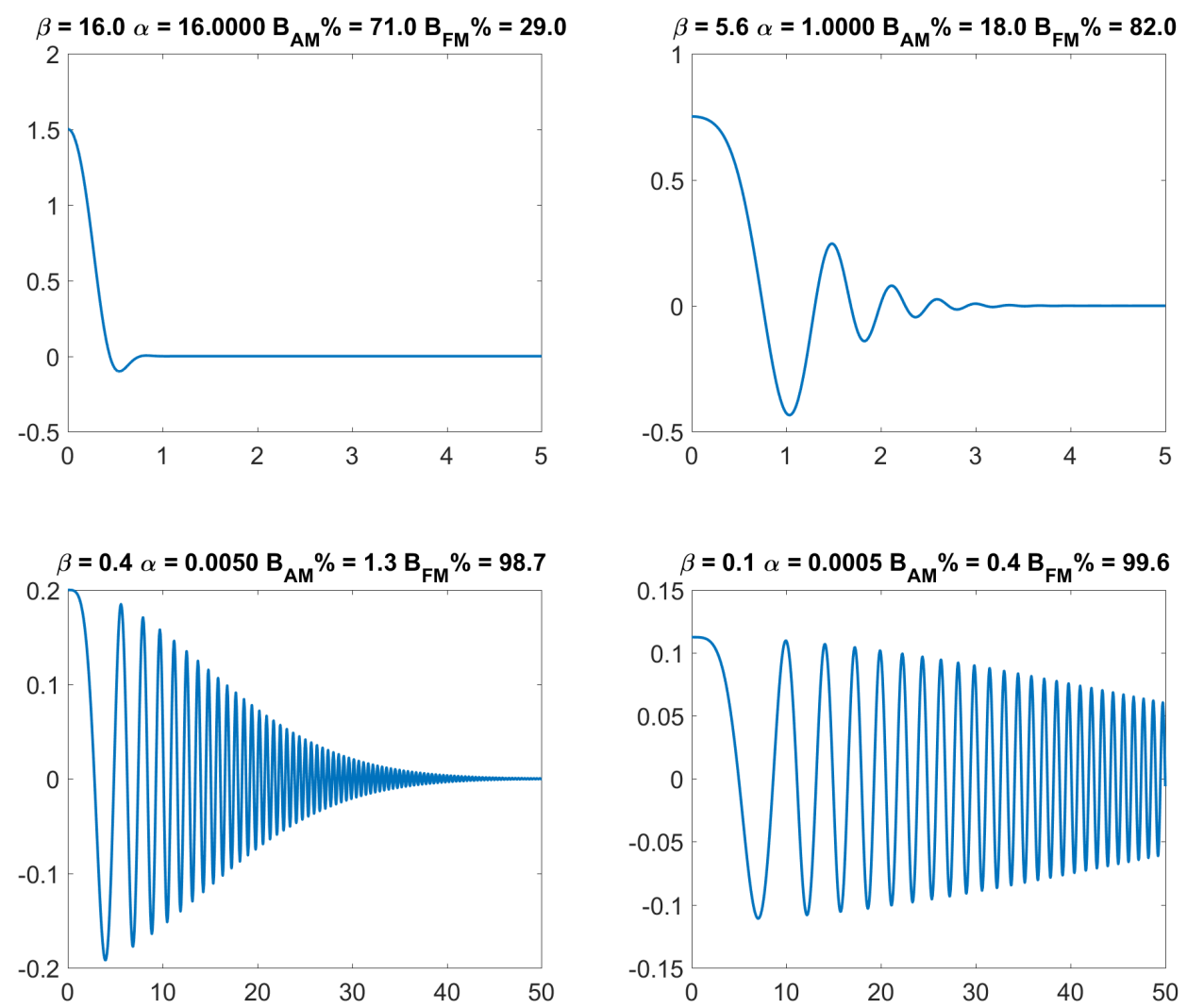

Figure 4.13: Illustration of different signals which all have identical bandwidth but varying levels of AM and FM contributions to bandwidth [10].

We can find the AM and FM contributions of the linear ramp type chirps as well. Here I have defined a piecewise treatment of the signal. I integrate the signal from time zero to the time at which the signal goes to 1 for the onset or to 0 for the offest. To normalize the signal's energy a constant was added.

The onset chirp is defined as:

$$
s(t)=\alpha t e^{\frac{j \beta t^{2}}{2}+j \omega_{0} t}
$$


An energy normalization constant was calculated:

$$
k_{\text {onset }}=\sqrt{\frac{1}{\alpha}}
$$

The envelope of the signal is:

$$
A(t)=\left(k_{\text {onset }} \alpha t\right)
$$

The phase term of the signal is:

$$
\phi(t)=\frac{1}{2} \beta j t^{2}+j t \omega
$$

AM bandwidth is calculated:

$$
B_{A M}^{2}=\int_{0}^{1 / \alpha} A^{\prime}(t)^{2} d t=\int_{0}^{1 / \alpha}\left(\frac{d\left(k_{\text {onset }} \alpha t\right)}{d t}\right)^{2} d t=\alpha
$$

The FM bandwidth is defined as:

$$
B_{F M}^{2}=\int\left(\phi^{\prime}-\langle w\rangle\right)^{2} A^{2} d t
$$

The derivative of the phase term is needed:

$$
\phi^{\prime}(t)=\frac{d}{d t}(\text { phase })=\frac{d}{d t}\left(\frac{\beta t^{2}}{2}+\omega t\right)=\beta t+\omega
$$

Here I take the same result as Cohen for mean frequency $\langle w\rangle$.

$$
\langle w\rangle=\int \phi^{\prime}(t) A^{2}(t) d t=\omega
$$


We evaluate the FM bandwidth:

$$
B_{F M}^{2}=\int_{0}^{1 / \alpha}(\beta t+\omega-\omega)^{2}\left(k_{\text {onset }} \alpha t\right)^{2} d t=\frac{\beta^{2}}{5 \alpha^{4}}
$$

The same analysis follows for the offset chirp.

The offset chirp is defined as:

$$
s(t)=(1-\alpha t) e^{\frac{j \beta t^{2}}{2}+j \omega_{0} t}
$$

An energy normalization constant was calculated:

$$
k_{\text {offset }}=\frac{1}{\sqrt{\frac{1}{\alpha}-2+\alpha}}
$$

The envelope of the signal is:

$$
A(t)=\left(k_{\text {offset }}(1-\alpha) t\right)
$$

The phase term of the signal is:

$$
\phi(t)=\frac{1}{2} \beta j t^{2}+j t \omega
$$

AM bandwidth is evaluated:

$$
B_{A M}^{2}=\int_{0}^{1 / \alpha}\left(\frac{d\left(k_{\text {offset }}(1-\alpha) t\right)}{d t}\right)^{2} d t=\alpha
$$

The FM bandwidth is defined as:

$$
B_{F M}^{2}=\int\left(\phi^{\prime}-\langle w\rangle\right)^{2} A^{2} d t
$$


The derivative of the phase term computes to:

$$
\phi^{\prime}(t)=\frac{d}{d t}(\text { phase })=\frac{d}{d t}\left(\frac{\beta t^{2}}{2}+\omega t\right)=\beta t+\omega
$$

Here I take the same result as Cohen for mean frequency $\langle w\rangle$.

$$
\langle w\rangle=\int \phi^{\prime}(t) A^{2}(t) d t=\omega
$$

We evaluate the FM bandwidth:

$$
B_{F M}^{2}=\int_{0}^{1 / \alpha}(\beta t+\omega-\omega)^{2}\left(k_{o f f s e t}(1-\alpha) t\right)^{2} d t=\frac{\beta^{2}}{5 \alpha^{4}}
$$

We can now look at tiling the time-frequency plane with many chirplets of many different durations, bandwidths, and rotations. The custom fovea will use these chirplets as the detectors for characterizing audio. A custom tiling of the timefrequncy plane will be used. An example of control of the time-frequency plane and arbitrary tiling is shown in Figure 4.14. The figure shows nine different chirplets occupying different parts of the time-frequency plane where each grouping has the same chirp but different window lengths. 


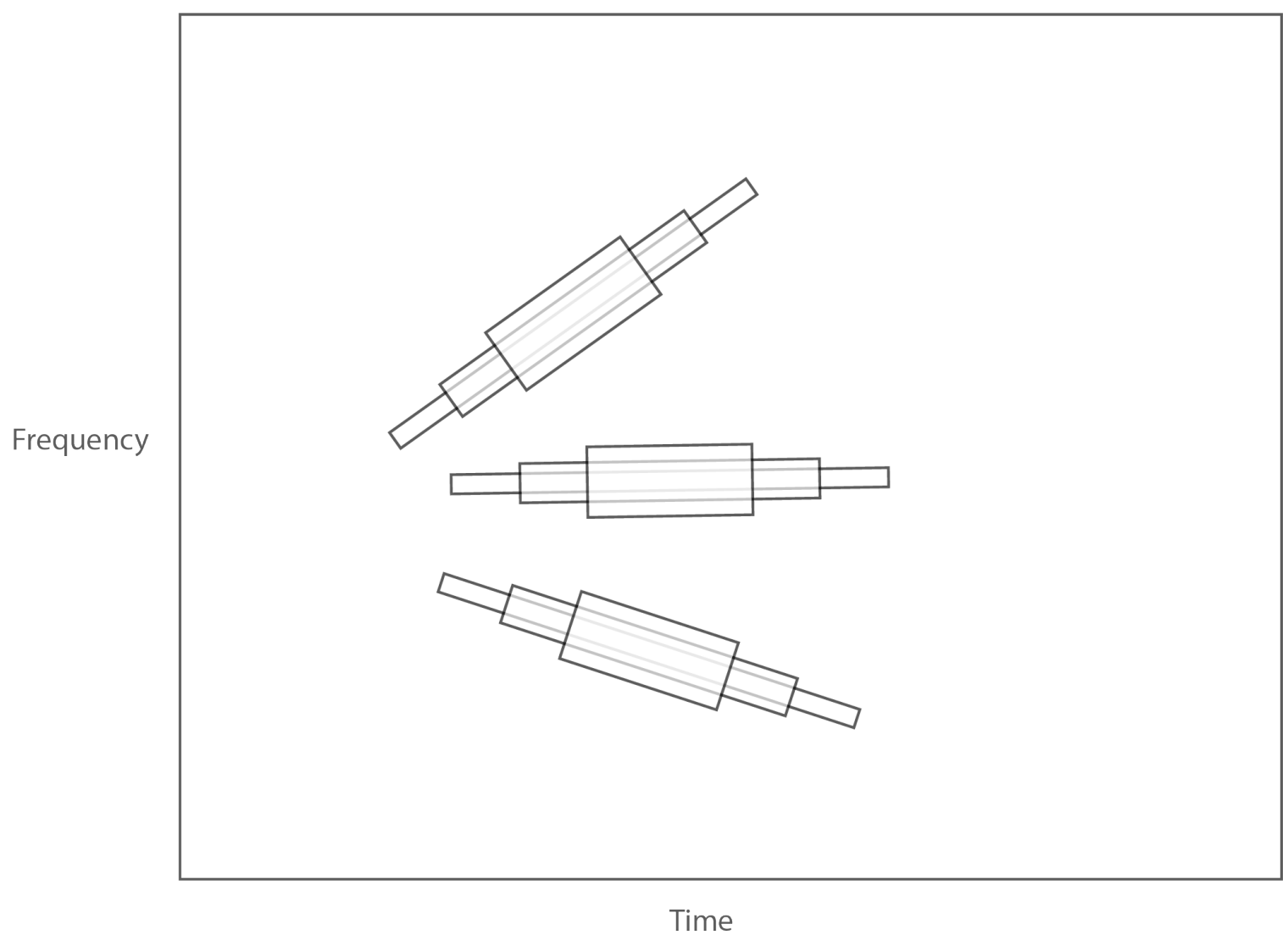

Figure 4.14: Arbitrary control of time-frequency tiling. Placement of the chirplets could be anywhere, but chirplets of similar rotations are grouped together to show varying window lengths.

\section{The Chirplet Deck}

We can now generate many different types of chirps and compare each to the signal under analysis. We tile the time-frequency plane with a chirplet "deck" using various time and frequency placements to match the signal under analysis at many different areas of the time-frequency plane. A chirplet deck is the terminology used to describe all the chirplets that are generated for a single point in the time-frequency plane. A tiling example of a arbitrary chirplet deck is show in Figure 4.15. 


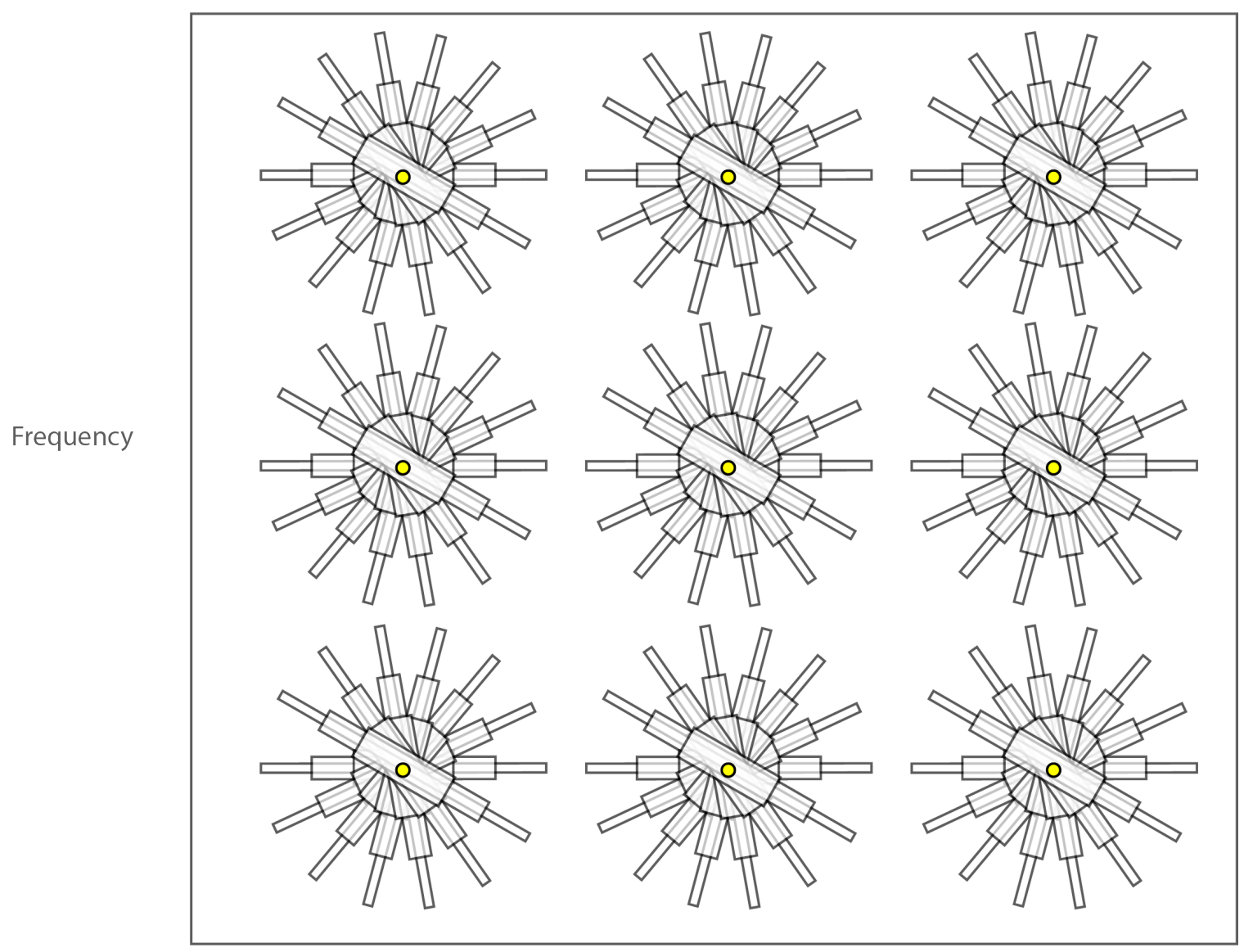

Time

Figure 4.15: Visualization of tiling the time-frequency plane with chirplet decks.

Notice how we are resolving different chirplet frequency slopes and chirplet lengths at each point in the time-frequency domain. Also notice in Figure 4.15 that the total area of the chirplet in the time-frequency plane varies as the signal becomes longer or shorter in line with the uncertainty principle. We trade bandwidth for temporal area in the plane. For a given setup of the front end we will specify a number of parameters which tell us about how we are tiling the domain with our chirplets and chirplet decks. We can specify the slopes of our chirplets by specifying a beginning slope and an end slope and a slope step. FM chirplets will be created between these two slopes for each deck in the time-frequency domain. FM is defined in 
terms of cycles per second per second or Hertz per second. When a chirplet is created, the frequency sweep used will follow this FM slope given the length of the chirplet. Thus a longer chirplet will have higher and lower ending frequencies to maintain a given slope than a shorter chirplet would. Chirplet decks in this project are made with respect of Nyquist. It was made sure, that given the targeted sampling rate of $44100 \mathrm{~Hz}$ for the project, that no chirplet contained frequency content above 22050 Hz. Chirplet lengths can also be specified and for each chirplet deck, all chirplets will be made in each of the specified lengths. Chirplet length is defined in seconds. For a chirplet deck, the user can specify the length of chirplets in seconds which should be made. Length and slope are illustrated in Figure 4.16

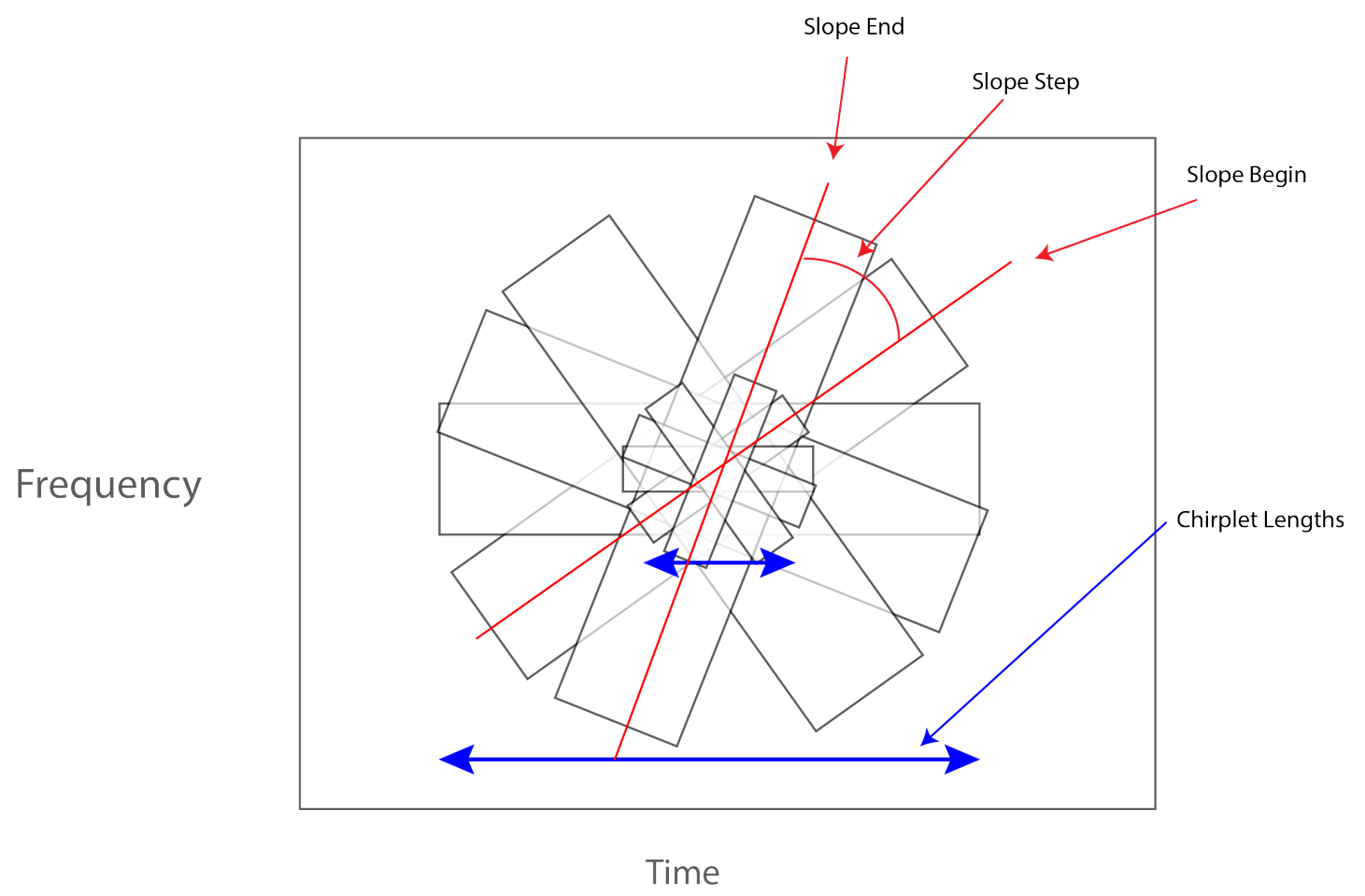

Figure 4.16: Slope and length chirplet deck parameters.

We can also specify where each deck is placed in the time-frequency plane. We 
assume a rectangular grid and specify how often along the time and frequency axis the decks are created. The signal being measured is evaluated every $\mathrm{N}$ samples dictated by the time axis step. The two parameters are illustrated in Figure 4.17 as shown between yellow dots which represent the deck centers. A rectangular grid is used in these examples, but other spatial patterns could be used and the spacings could be nonuniform allowing further foveation to occur.

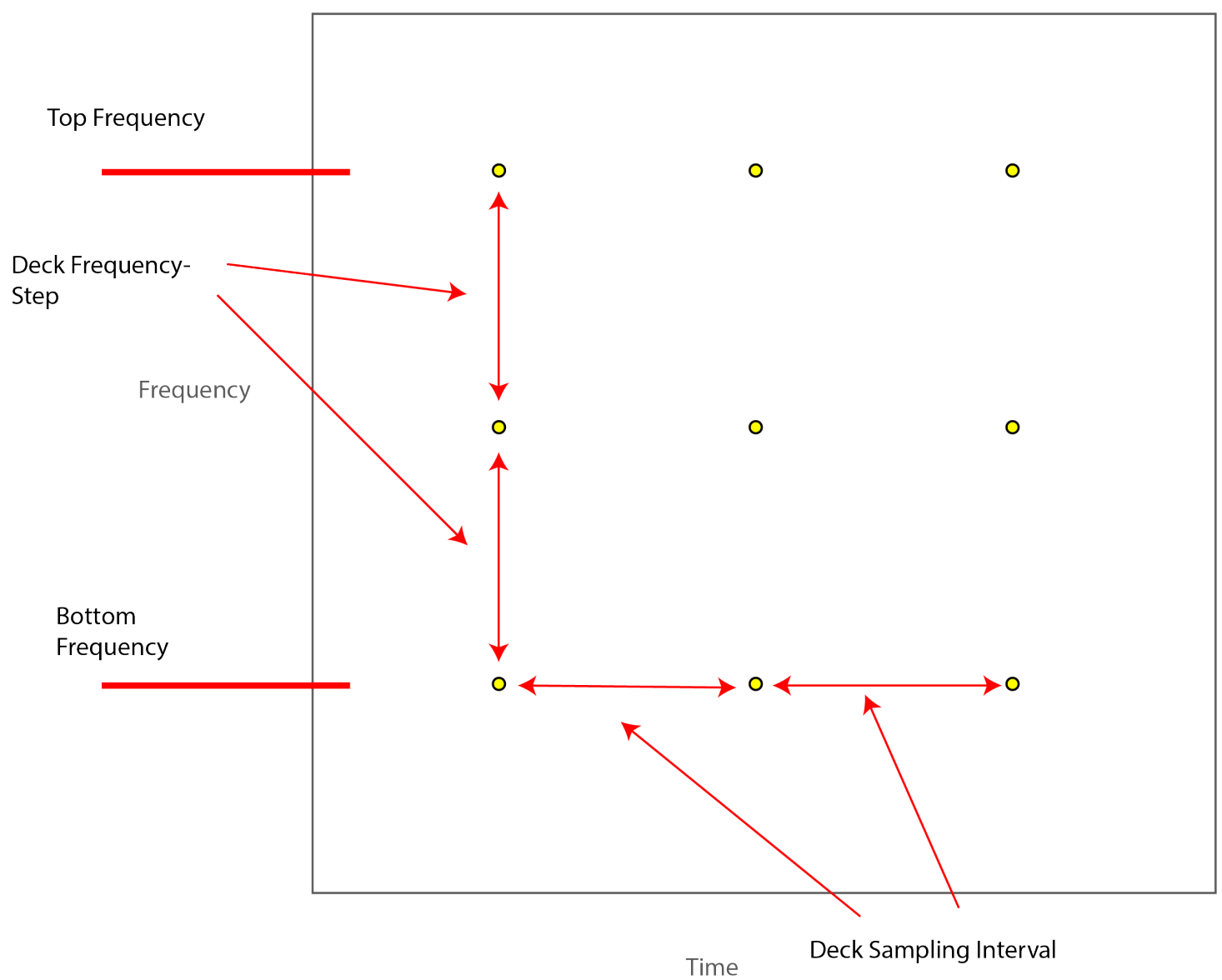

Figure 4.17: Time and frequency step chirplet deck parameters. Each yellow dot represents a chirplet deck as was the case in Figure 4.15. 


\section{Custom Foveation of the Time-Frequency Plane}

These chirplets can now be tiled to create many different resolutions of the timefrequency plane. A point in time and frequency can be specified and a deck with an arbitrary number of chirplets can analyse the signal at the time-frequency location. A foveation or resolution of the front end can now be examined. Areas of great importance for detection can be tiled or foveated with high resolution. An area of the time-frequency domain which is of less importance can have less resources dedicated to it. This allows an analysis front end to be customizable to the signal types being analyzed. This mimics examples in biology which dedicate resources to particularly relevant signals. Adding resources where needed and taking away resources where they are not is hypothesized to aid identification and back end algorithm learning efficiency.

We have identified two different types of foveation or resolution of the custom front end. We have identified a spatial foveation wherein a deck is stamped tighter along either the time or frequency axis. Again, the deck refers to all the differing types of chirplets evaluated at a particular point in the time-frequency plane. We have also identified a chirp deck resolution. In a high chirp deck foveation, the types of chirps used is many. This chirp type amount can come from either a large amount of chirp lengths or slopes or amplitude profiles.

Spatial resolution in the time-frequency plane is demonstrated in Figure 4.18. Here we see high versus low spatial resolution if each yellow dot represents a chirplet deck. However we can also look at a low versus high deck resolution which is demonstrated in Figure 4.19 versus Figure 4.20. We see the difference between many chirplets being evaluated versus few. 


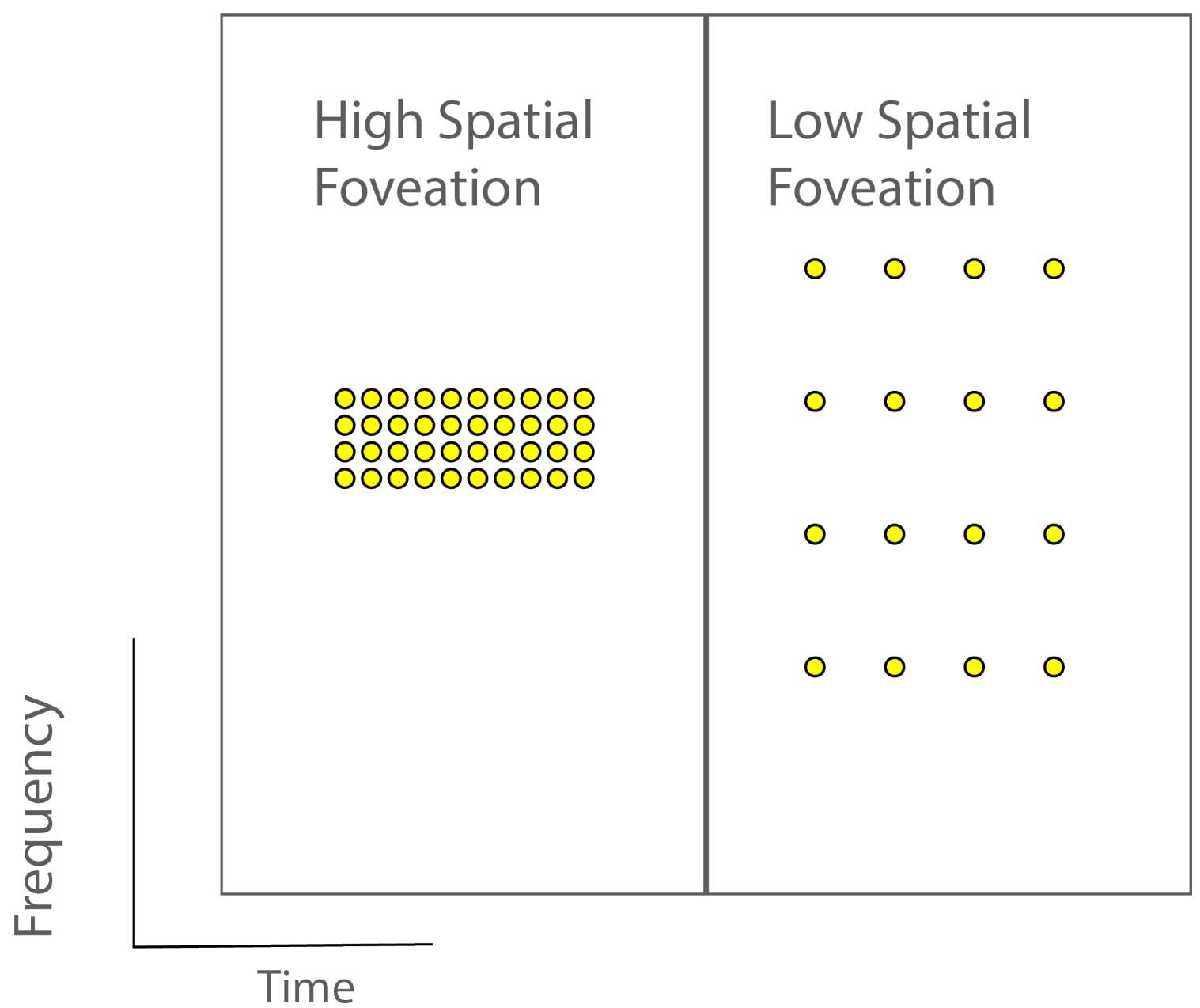

Figure 4.18: High and low spatial foveation time frequency plane. Each point in the figure portrays a chiplet deck center evaluation point. On the left we evaluate much more finely in time and frequency. 


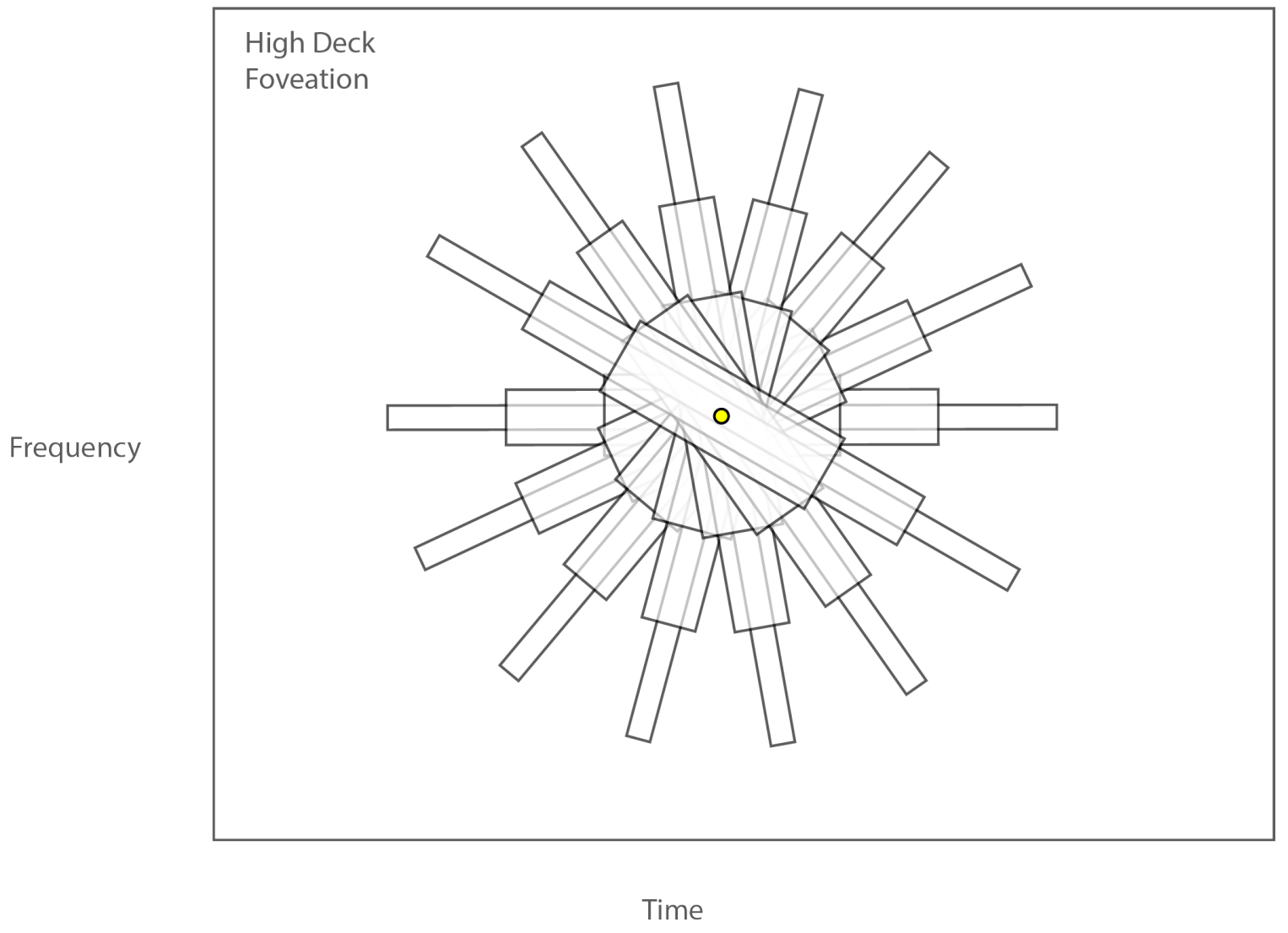

Figure 4.19: High foveation of chirplet deck. Here we see multiple chirplet lengths and slopes comprising high deck foveation. 
Frequency

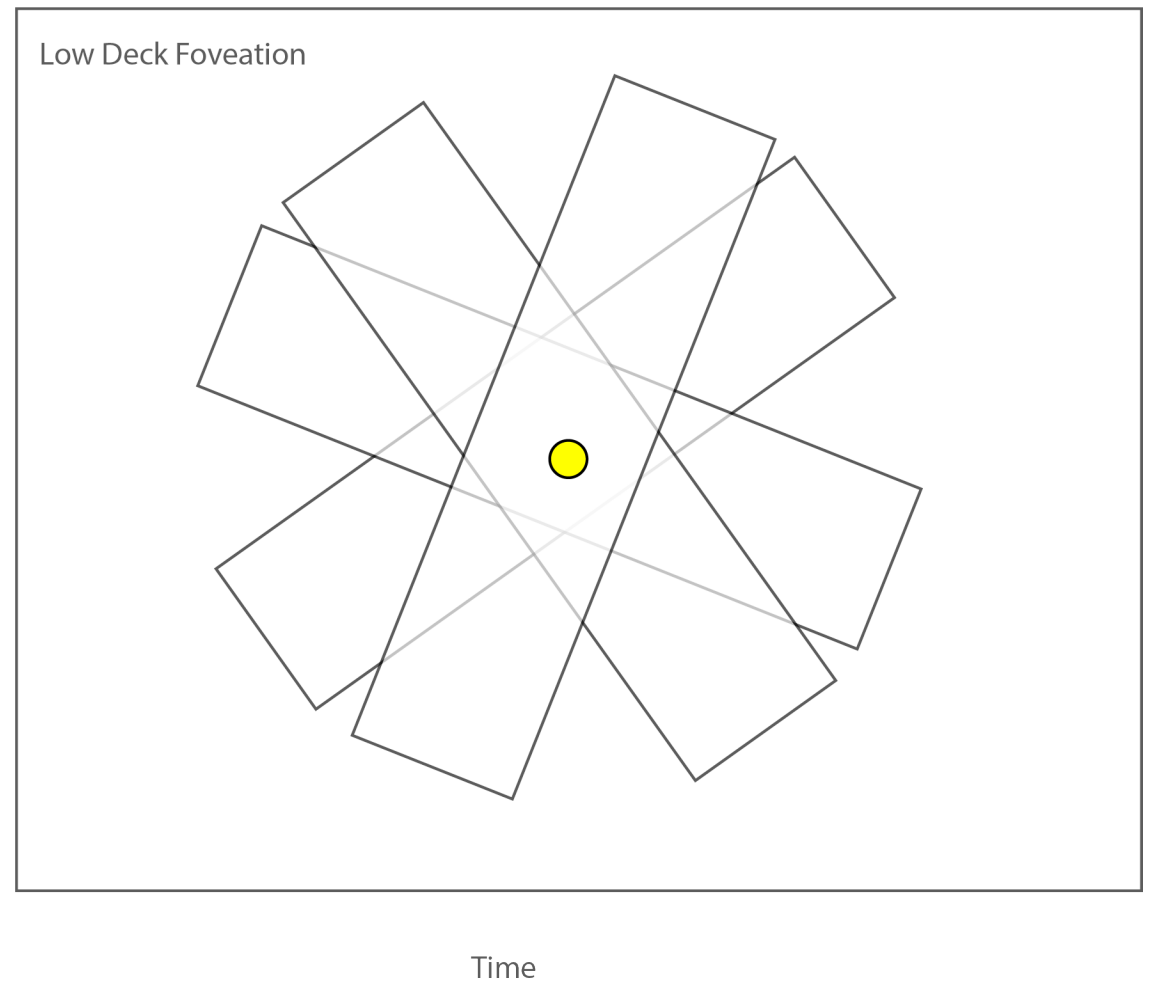

Figure 4.20: Lower foveation of chirplet deck. The chirplet deck does not have as many lengths or slopes, comprising low deck foveation.

\section{Distance Metric}

To match the chirplet against a given signal, a distance metric between the chirp waveform and the signal must be used. We will use a normalized dot product is used. However, other metrics could be used. The normalized dot product is shown below.

$$
\text { similarity }=\cos (\theta)=\frac{A \cdot B}{|A|_{2}|B|_{2}}=\frac{\sum_{i=1}^{n} A_{i} B_{i}}{\sqrt{\sum 1_{i=1}^{n} A_{i}^{2}} \sqrt{\sum 1_{i=1}^{n} B_{i}^{2}}},
$$

This metric runs from 1 to -1 . A 1 is a perfect match. A -1 indicates a perfect opposite. 
The Guassian modulated chirp is again defined as:

$$
s(t)=(\alpha / \pi)^{\frac{1}{4}} e^{-\frac{\alpha t^{2}}{2}+\frac{1}{2} \beta j t^{2}+j t \omega}
$$

The mathematical analysis included in this chapter is done in continuous time. The artificial accousic fovea however is implemented and programmed in discrete time. The discrete signals programmed do not map one to one to the continuous time descriptions and this needs to be acknowledged. A best approximation is approached in discrete time using the Matlab implementation of the front end. The chirplets are generated using a Matlab equivalent of the paramterized signal definition shown in Equation 4.32 and sampled at the project sample rate of $44100 \mathrm{~Hz}$. An illustration of the sampling which is occuring is shown in Figure 4.21. The implementation is framed using the minimum time-frequency signal and the uncertainty principle of continuous time while implemented in discrete time. 


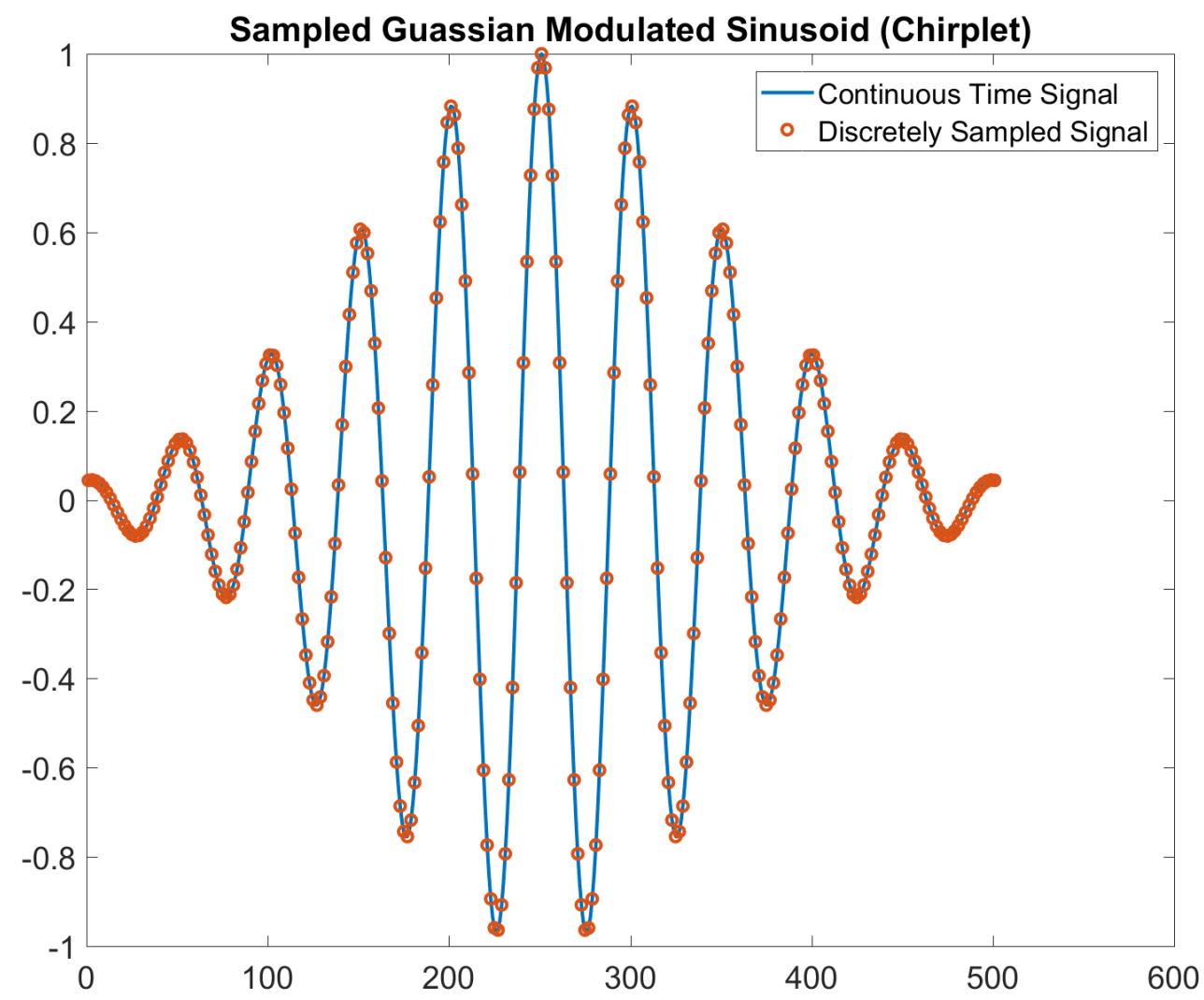

Figure 4.21: A continuous time chirp signal is discretely sampled for the Matlab implementaiton of the artificial accousic fovea. 


\section{SIGNAL PROCESSING USING THE ARTIFICIAL ACOUSTIC FOVEA}

We start with a visualization of the chirplet deck matching process. A deck of chirps was used with the normalized dot product on a Marmoset trill call. For each point in the time-frequency plane, a total of 3 downward sloping chirplets, 3 upward sloping chirplets and 1 constant chirplet was compared to the signal. The slopes were $0 \mathrm{~Hz} / \mathrm{sec}, 100 \mathrm{~Hz} / \mathrm{sec}, 600 \mathrm{~Hz} / \mathrm{sec}$ and $1100 \mathrm{~Hz} / \mathrm{sec}$. Each of these 4 chirplets was constructed in two lengths, .01 seconds and .005 seconds. Negative sloping chirplets are constructed for each positive sloping chirplet. The chirplet deck was then translated throughout time and frequency and compared to the signal at that time-frequency point. An illustration of this translation and chirplet array is shown in Figure 5.1. Of note is that only Guassian modulated chirps and not ramp modulated chirps are being used in the deck. 


\section{Frequency}

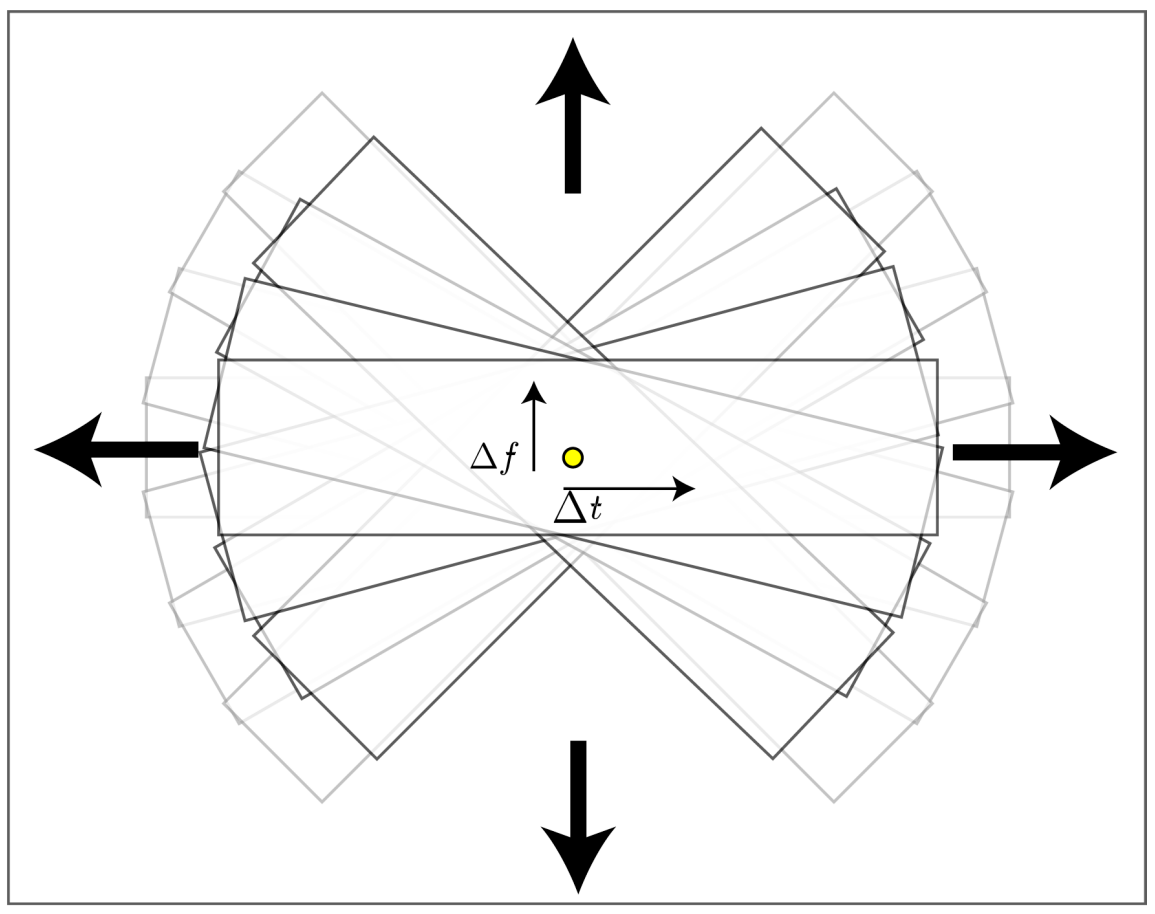

Time

Figure 5.1: Translation of the chirplet deck in time and frequency.

Figure 5.1 shows a deck with 14 different chirplet types. In the chirplet front end programmed in Matlab, a structure was created that defines the chirplet deck we create to move in the time-frequency plane and match against the signal. The parameters of the structure which dictate the spatial foveation and the deck foveation are presented in Table 5.1. That structure is passed to a MATLAB function, chirplets_f() which creates all chirplet signals. That structure as viewed in Matlab is shown in Figure 5.2.

\section{Chirplet Analysis}

To begin visualization using the fovea, we first look at the output of a single chirplet across the time-frequency plane. To help illustrate the tiling used, Figure 
5.3 uses $500 \mathrm{~Hz}$ spacing between $500 \mathrm{~Hz}$ and $20 \mathrm{kHz}$ on the frequency axis and time interval steps of 1000 points (22.7 ms at a $44100 \mathrm{~Hz}$ sampling rate.) A lower timefrequency spacing is being used. We are using a low spatial foveation and a low deck foveation, as defined by deck parameters in Table 5.1. We can observe the low resolution and the result in Figure 5.3. This is a single chirplet with $-1100 \mathrm{~Hz}$ slope with a .005 second duration matching the signal under test.

Table 5.1: Example foveation. Very low spatial and low deck foveation.

$\begin{array}{ll}\text { Chirp Lengths } & {[.005 .01] \mathrm{sec}} \\ \text { Begin Slope } & 100 \mathrm{~Hz} / \mathrm{sec} \\ \text { End Slope } & 1100 \mathrm{~Hz} / \mathrm{sec} \\ \text { Slope Step } & 500 \mathrm{~Hz} / \mathrm{sec} \\ \text { Sampling Interval } & 1000 \mathrm{Samples} \\ \text { Frequency Start } & 500 \mathrm{~Hz} \\ \text { Frequency End } & 20000 \mathrm{~Hz} \\ \text { Frequency Step } & 500 \mathrm{~Hz} \\ \text { Total Chirps } & 14\end{array}$




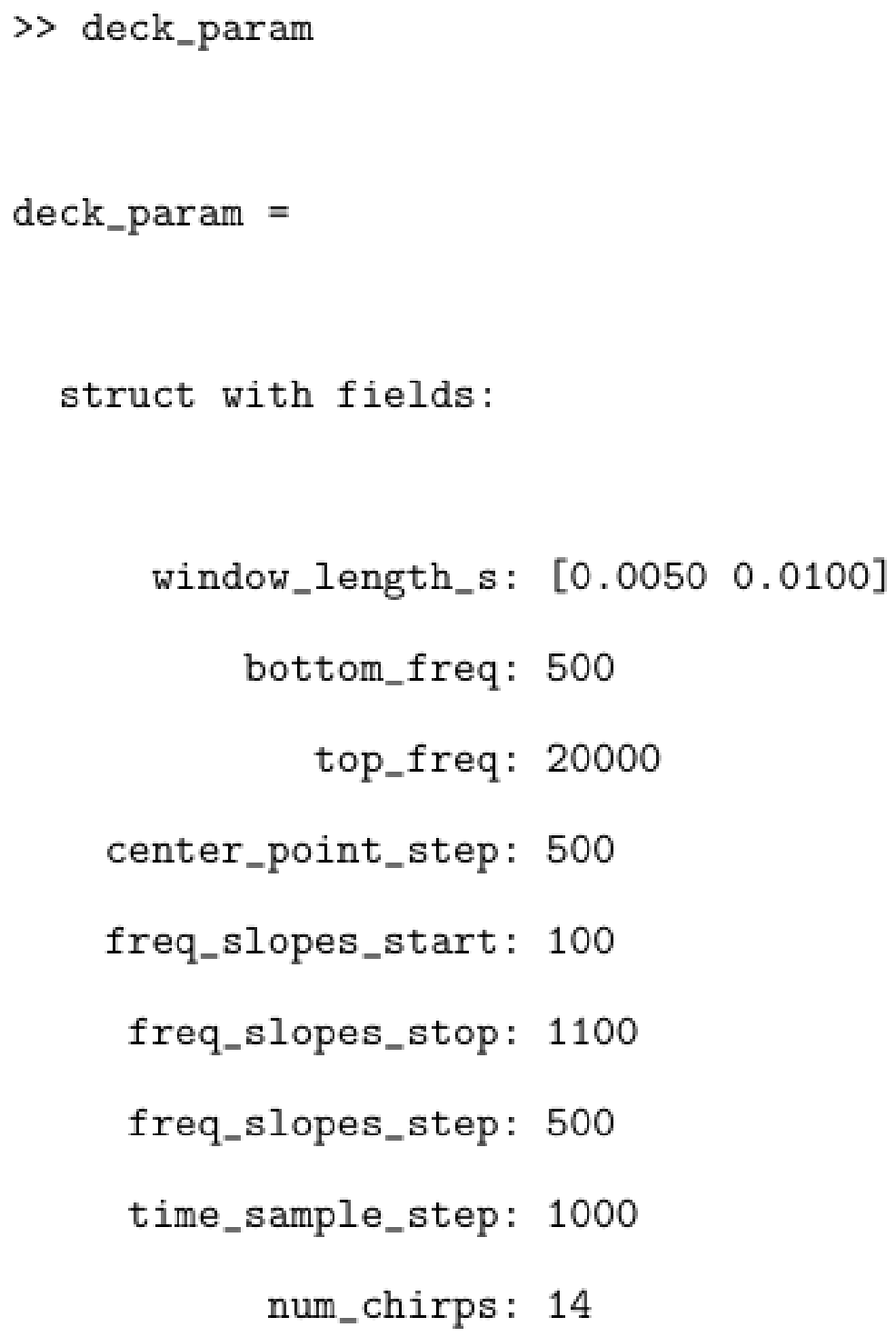

Figure 5.2: Matlab structure of chirplet deck parameters. 

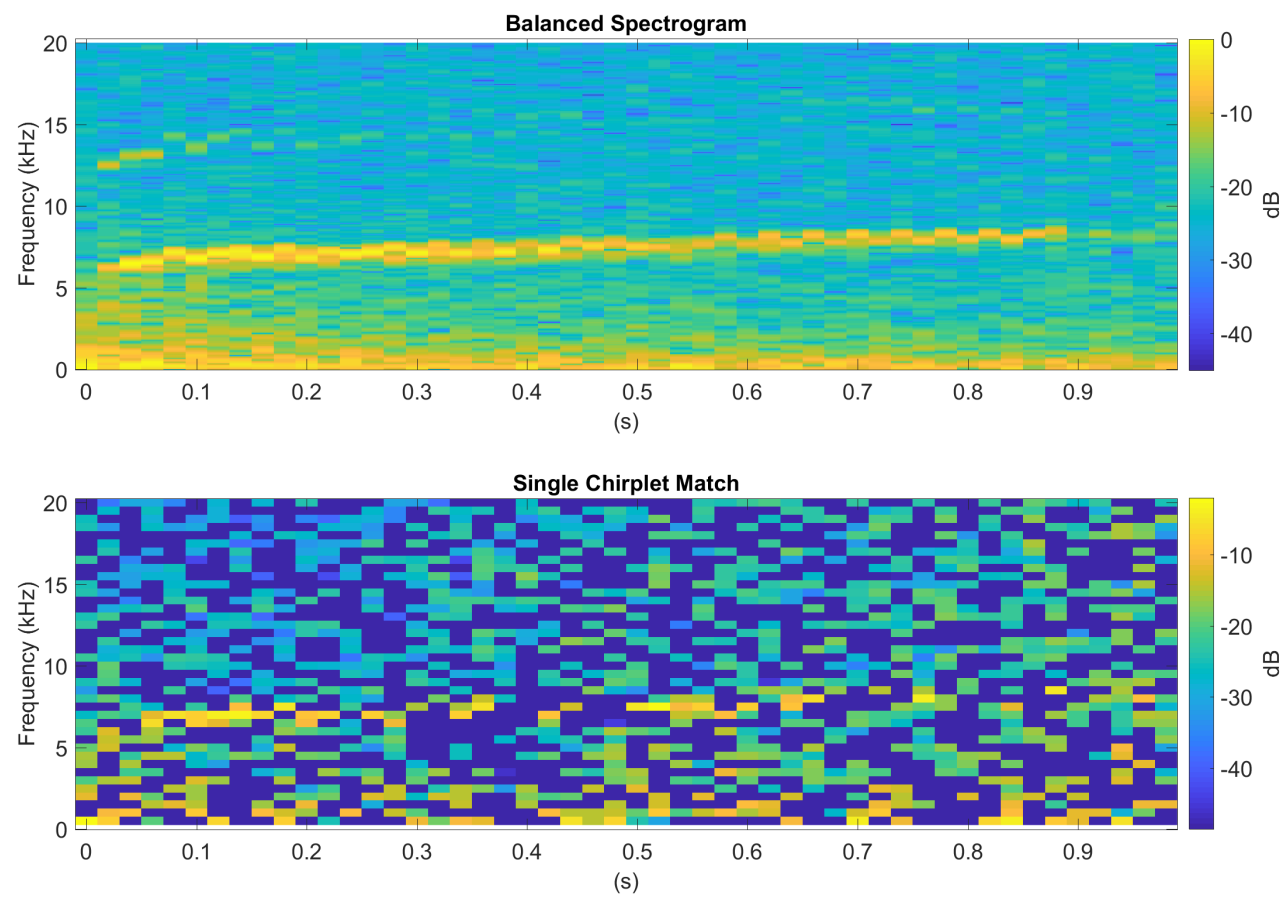

Figure 5.3: $-1100 \mathrm{~Hz} / \mathrm{sec}$ sloping chirplet .005 seconds match. Low spatial foveation. Chirplet deck parameters are shown in Table 5.1. The spectrogram window used was: Balanced: .005 Seconds.

We see that this single chirp is indeed sensitive and activated along the signal of interest and is matching the signal best at specific points. The match is indicated by smaller negative yellow values of the bottom portion of Figure 5.3.

\section{High Spatial Foveation}

What if we want to see the chriplet matching at more points along time and frequency? This is an increase in spatial resolution or foveation but not deck foveation. We can examine this same chirplet every 50 samples of the signal and using $50 \mathrm{~Hz}$ center frequency spacing between $500 \mathrm{~Hz}$ and $20000 \mathrm{kHz}$ as shown in Figure 5.4. So far we are only examining output from one specific chirplet for illustration. The complete front end outputs at each point in the time-frequency plane a total of 14 
different chirplet matches as defined by our deck but thus far we have restricted visualzation of only one chirplet. The full deck output will be used shortly. The same deck of chirplets is being used when we increase spatial foveation, but compared at more points along time and frequency. The associated deck parameters are defined in Table 5.2 . A spectrogram of the signal is shown above the chirplet detector front end output for comparison purposes. The normalized dot product metric is being used. Negative results from the distance metric are being set to 0 before visualization. We can zoom in on the output as shown in Figure 5.5.
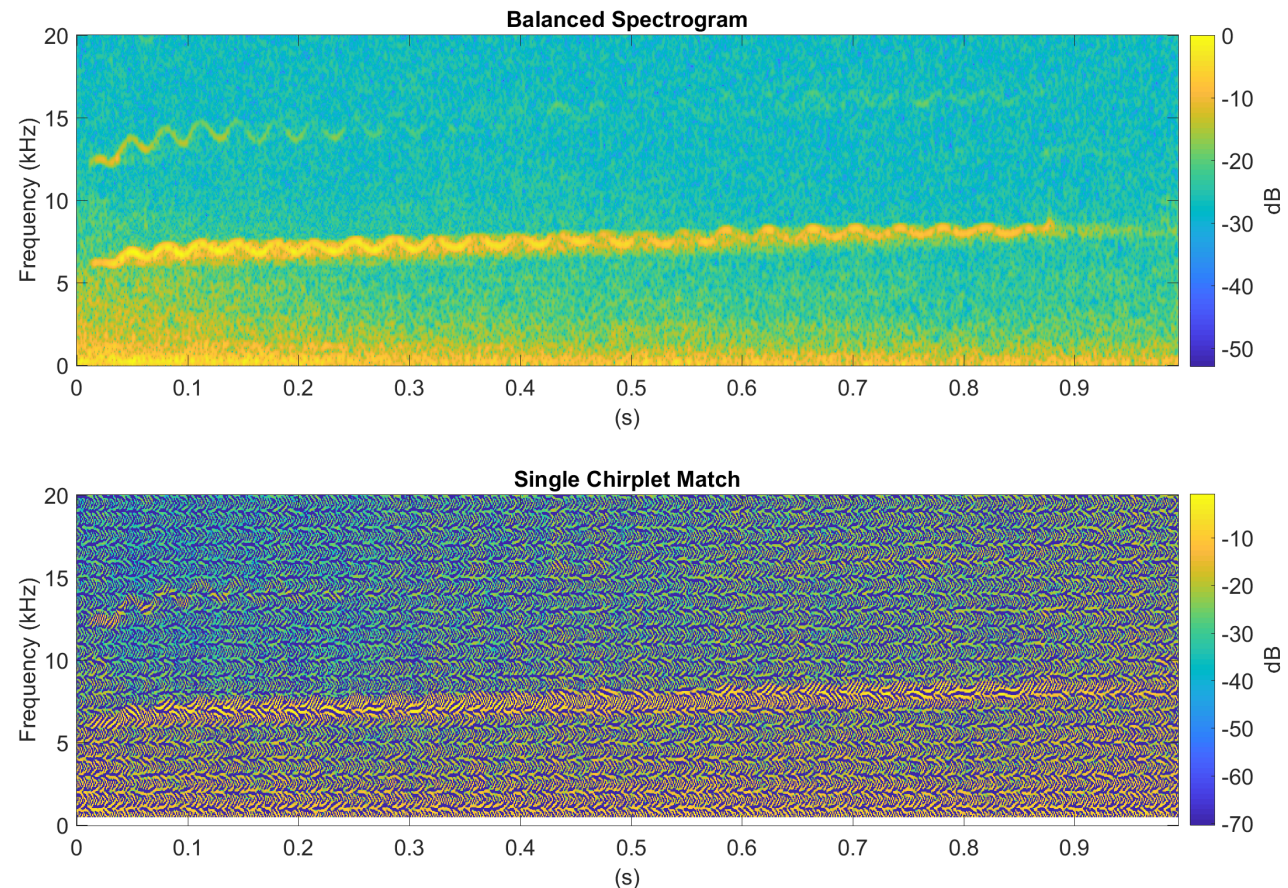

Figure 5.4: $-1100 \mathrm{~Hz} / \mathrm{sec}$ sloping chirplet .005 seconds match. High spatial foveation. Chirplet deck parameters are shown in Table 5.2. The spectrogram window used was: Balanced: .005 Seconds. 

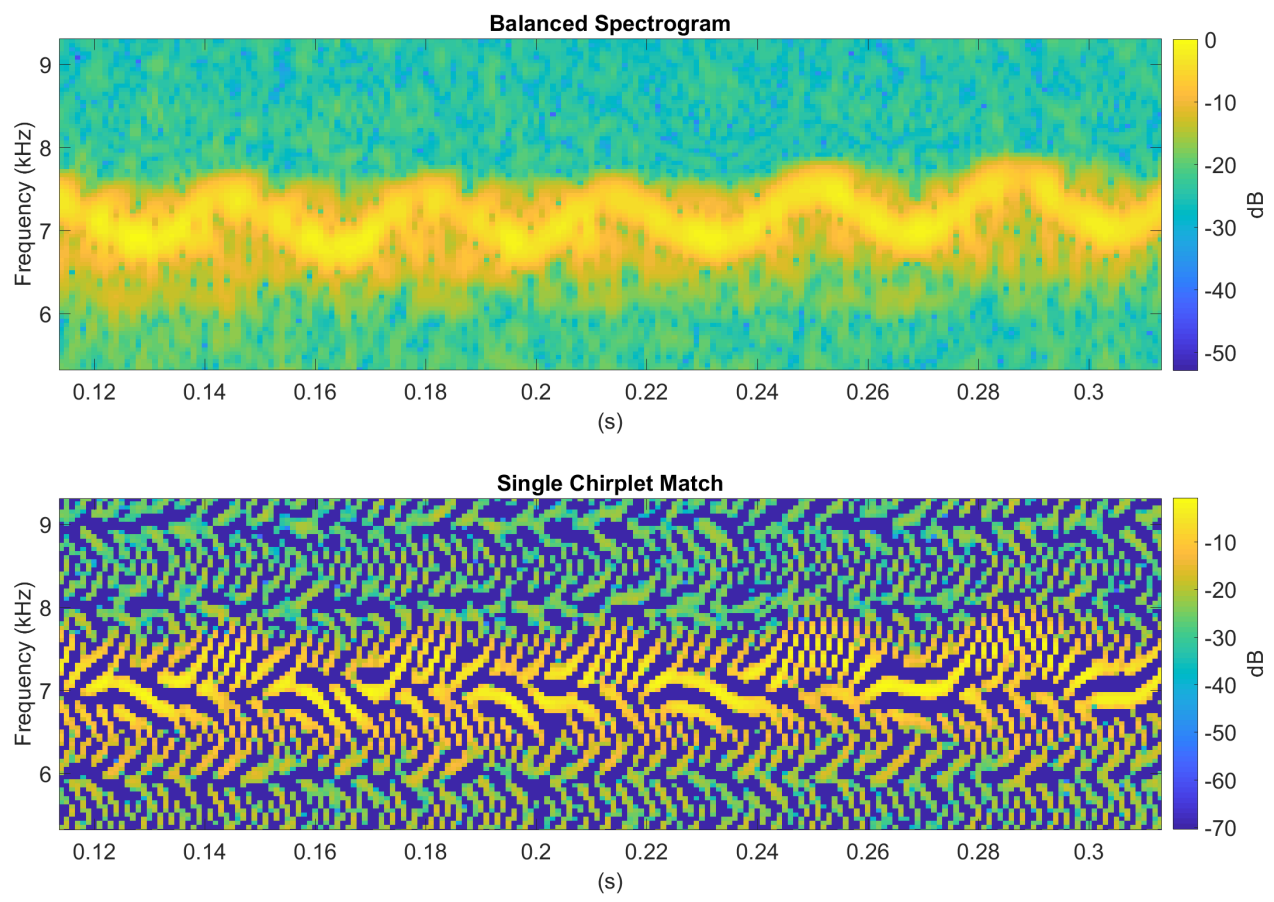

Figure 5.5: $-1100 \mathrm{~Hz} / \mathrm{sec}$ sloping chirplet .005 seconds match. High spatial foveation closer. Chirplet deck parameters are shown in Table 5.2. The spectrogram window used was: Balanced: .005 Seconds.

Table 5.2: Example foveation. High spatial foveation and low deck foveation.

$\begin{array}{ll}\text { Chirp Lengths } & {[.005 .01] \mathrm{sec}} \\ \text { Begin Slope } & 100 \mathrm{~Hz} / \mathrm{sec} \\ \text { End Slope } & 1100 \mathrm{~Hz} / \mathrm{sec} \\ \text { Slope Step } & 500 \mathrm{~Hz} / \mathrm{sec} \\ \text { Sampling Interval } & 50 \mathrm{Samples} \\ \text { Frequency Start } & 500 \mathrm{~Hz} \\ \text { Frequency End } & 20000 \mathrm{~Hz} \\ \text { Frequency Step } & 50 \mathrm{~Hz} \\ \text { Total Chirps } & 14\end{array}$


From this result, we can see that the chirplet is best matching the signal at very specific points. The amount of information on display here is greater than the corresponding spectrogram. At each point in the time-frequency plane we have information from $\mathrm{N}$ different chirplets describing how the signal is changing at timefrequency points. This is providing us distinct information about the signal at a particular point in the time-frequency plane, much more than a spectrogram can using its $\mathrm{N}$ basis functions [36]. The deck can contain the chirplet that provides the same information as the spectrogram. This information will be passed to a standard machine learning application later in the dissertation.

\section{$\underline{\text { Chirplet Deck Analysis }}$}

We can create a display showing the best match of the chirplets in each deck for each point in the time-frequency plane. We now longer are looking at just one chirp of the deck but the best of the whole deck. We are using a max operator to find the chirplet that best matches the signal at the particular point in the time-frequency plane. Matching here is describes as more positive $\mathrm{dB}$ values and associated colors of the graphics signifying higher match. The dot product result was converted to $\mathrm{dB}$ for increased range using $d B=10 \log _{10}$ (dotproduct). This result is shown in Figure 5.6. We see that there are parts of the signal which are not matching well. We can zoom in to further reveal locations where no chirps match the signal as shown in Figure 5.7. This example shows that over the fourteen different types of chirplets, we are not matching the signal completely along its entirety. 

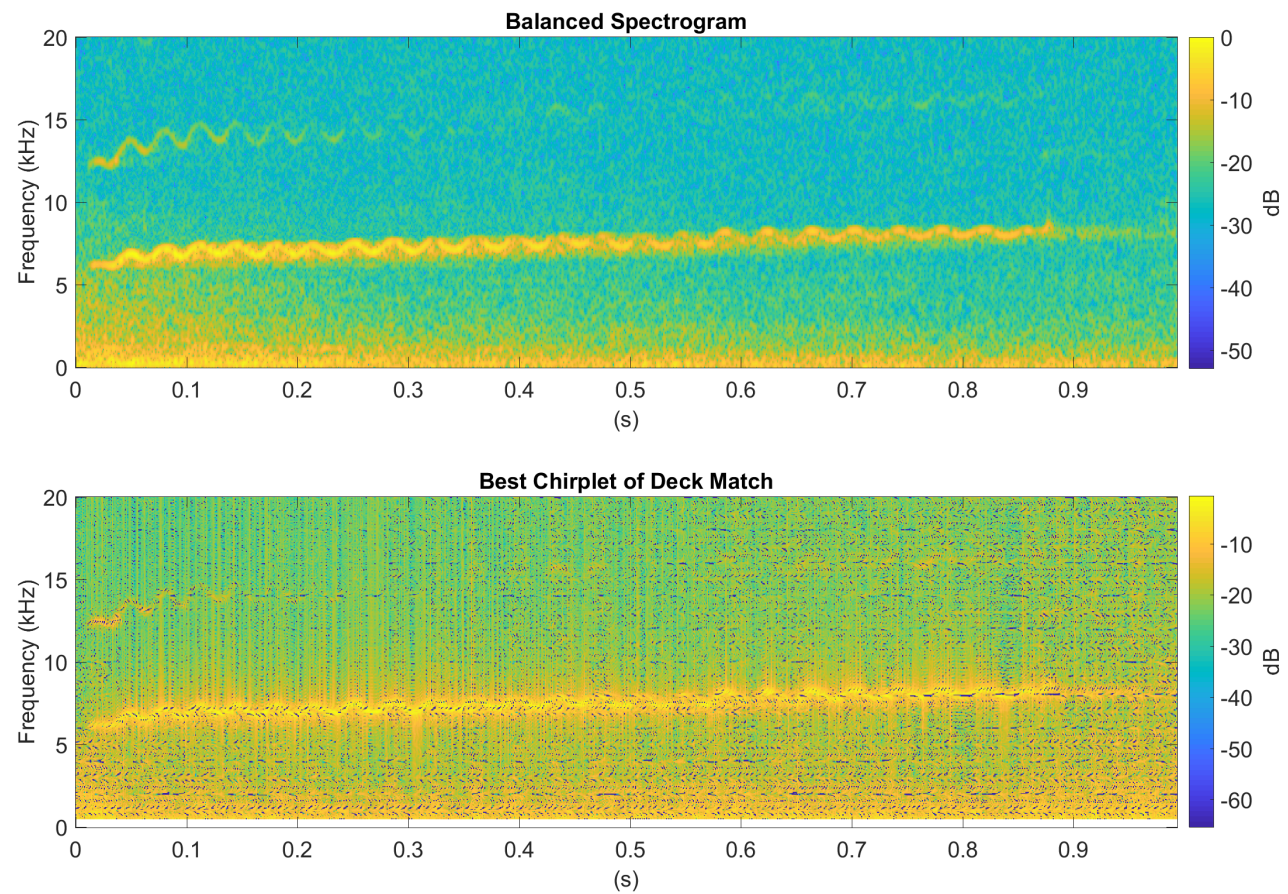

Figure 5.6: Overall best chirp output for high spatial foveation and low deck foveation. Chirplet deck parameters are shown in Table 5.2. The spectrogram window used was: Balanced: .005 Seconds. 

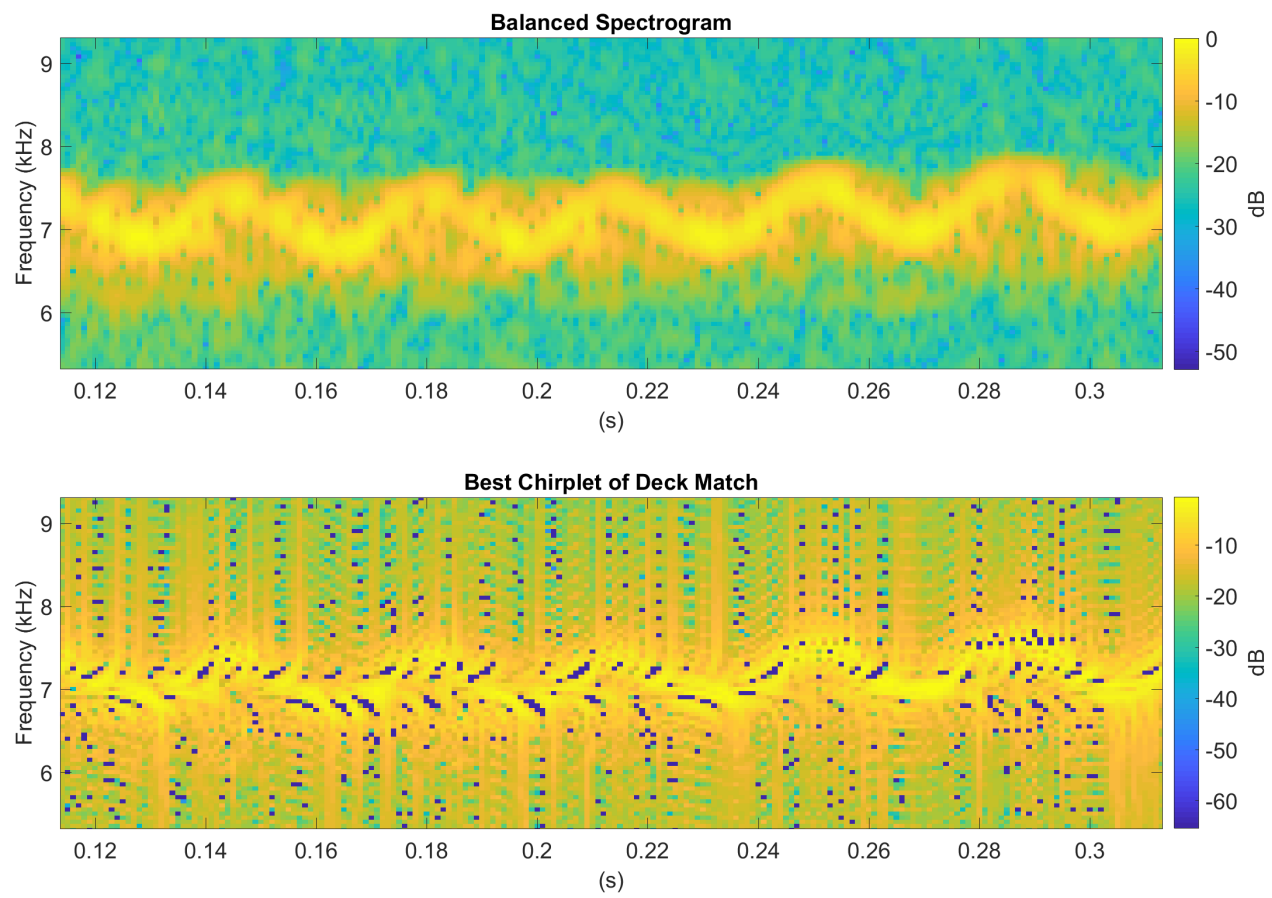

Figure 5.7: Overall best chirp output for high spatial foveation and low deck foveation zoomed. Chirplet deck parameters are shown in Table 5.2. The spectrogram window used was: Balanced: .005 Seconds.

\section{$\underline{\text { High Deck Foveation }}$}

How can we better match more parts of the signal? Let us increase the deck foveation to have more chirplets to match against. Spatial foveation is already high, but we still aren't matching parts of the signal as illustrated by blue low match areas of Figure 5.6. To correct this, we can add more chirp types to our chirp deck, thus increasing deck fovation. Let us start at 10,000 Hz/sec and step to 250,000 Hz/sec at 25,000 Hz/sec intervals. This new deck parameter setting is illustrated in Table 5.3. We have changed the total number of chirps at a point from 14 to 38 . We have also vastly increased the slope range of our chirplet deck. The chirp deck is now able to match the signal as better shown in Figure 5.8 and zoomed in on Figure 5.9. 

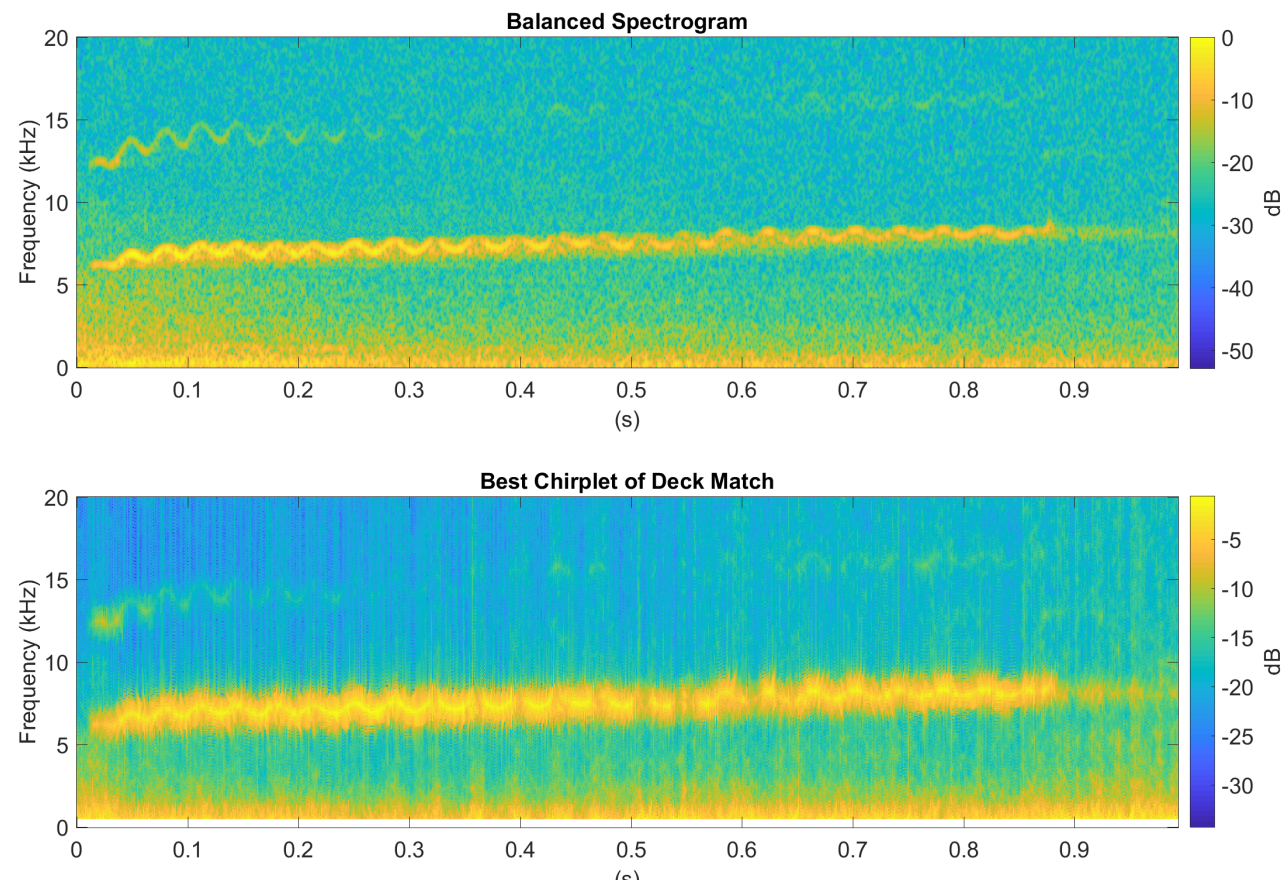

Figure 5.8: Overall best chirp output for a high deck foveation and high spatial foveation. Chirplet deck parameters are shown in Table 5.3. The spectrogram window used was: Balanced: .005 Seconds. 

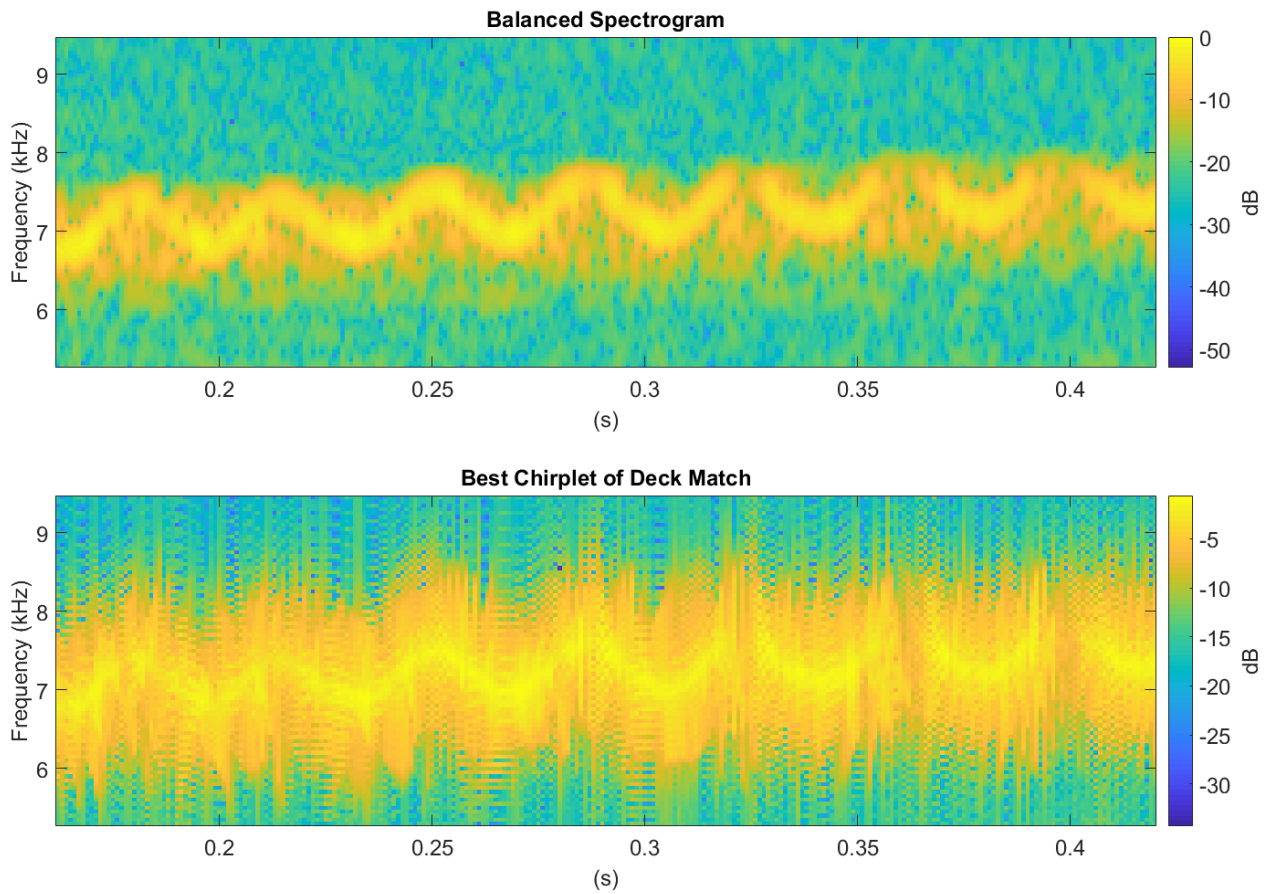

Figure 5.9: Overall best chirp output zoom for a higher deck foveation and high spatial foveation. Chirplet deck parameters are shown in Table 5.3. The spectrogram window used was: Balanced: .005 Seconds.

Table 5.3: Example foveation. High spatial foveation and high deck foveation.

$\begin{array}{ll}\text { Chirp Lengths } & {[.005 .01] \mathrm{sec}} \\ \text { Begin Slope } & 10000 \mathrm{~Hz} / \mathrm{sec} \\ \text { End Slope } & 250000 \mathrm{~Hz} / \mathrm{sec} \\ \text { Slope Step } & 25000 \mathrm{~Hz} / \mathrm{sec} \\ \text { Sampling Interval } & 50 \mathrm{Samples} \\ \text { Frequency Start } & 500 \mathrm{~Hz} \\ \text { Frequency End } & 20000 \mathrm{~Hz} \\ \text { Frequency Step } & 50 \mathrm{~Hz} \\ \text { Total Chirps } & 38\end{array}$


These results show us that given the current tiling we are able to achieve good matches across all parts of our signal when we increase both types of identified foveations, deck and spatial. There are not many parts of the signal which we are not able to match well at all. Jumping back to the output of a single chirp given high foveations, let us look at the constant slope chirp. Its results are similar but shifted slightly to match different areas of the signals. The following result is for the zero slope chirp which does not change frequency, i.e., it occupies a non-rotated rectangle in the time-frequency domain. The constant chirp result is shown in Figure 5.10.
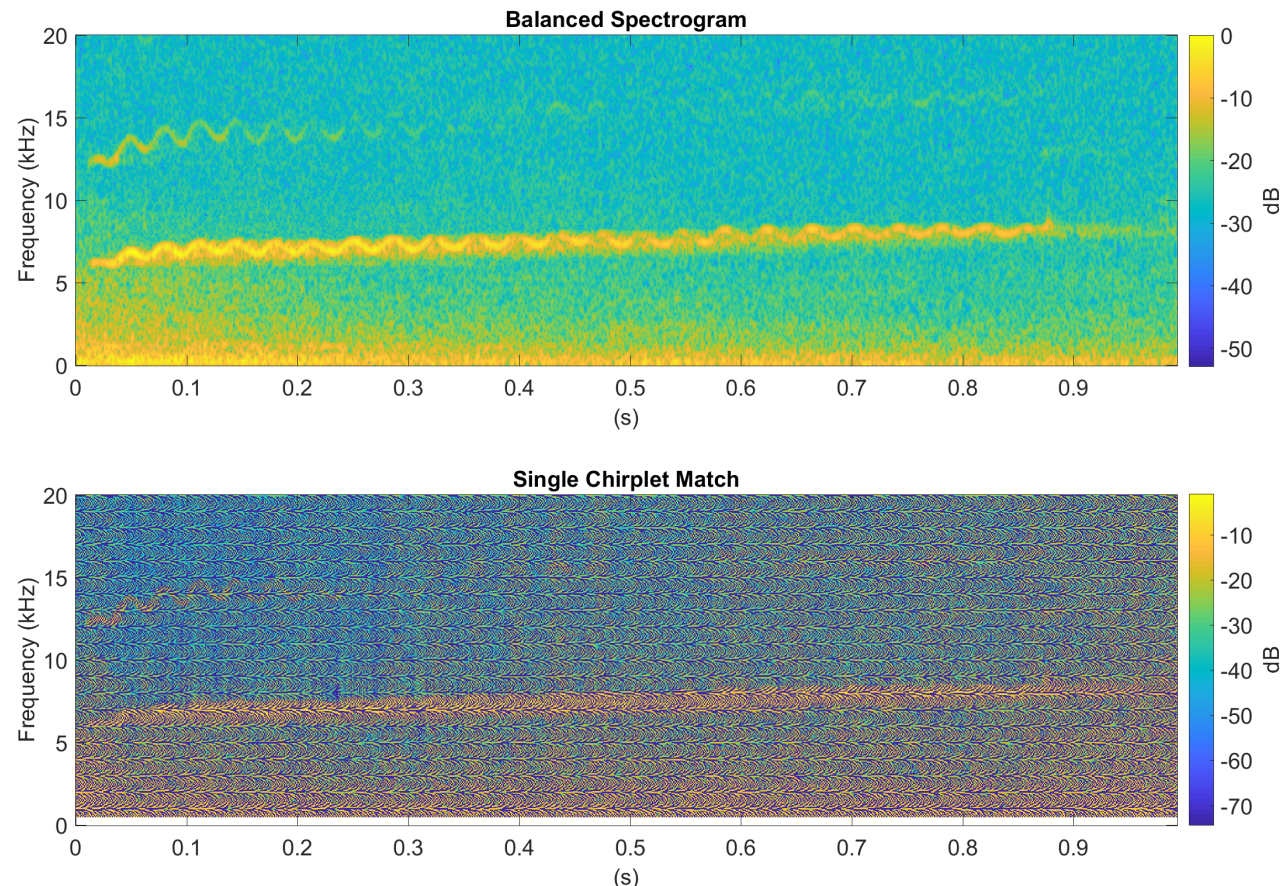

Figure 5.10: Constant chirp match results. Chirplet deck parameters are shown in Table 5.3. The spectrogram window used was: Balanced: .005 Seconds.

These results demonstrate the chirplet deck matching parts of the signal under examination. This demonstration shows how a deck is sensitive to certain parts of a signal as well as how the resolution of the scanning can be changed. Low resolution 
and high resolution detection was demonstrated in the spatial and deck senses. The chirplets do indeed match to the signal. Output for one specific time-frequency event was demonstrated in the results for a specific chirplet type. Let us look at a number of other Marmoset call types to show how the method works in these cases. We will keep the high spatial and high deck foveations of Table 5.3 for these examples. A phee Marmoset call match is shown in Figure 5.11. A twitter Marmoset call analysis is shown in Figure 5.12. Notice how we again are matching the signals well. Furthermore we have more local information than what the spectrogram provides us. 


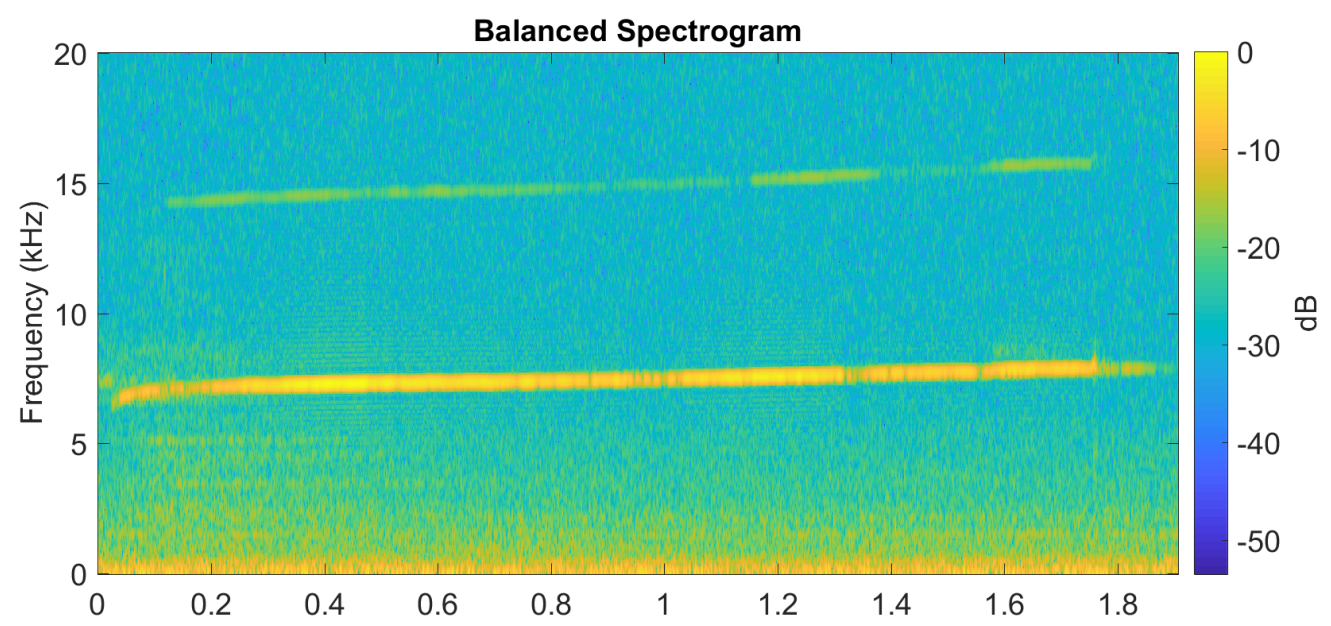

(s)

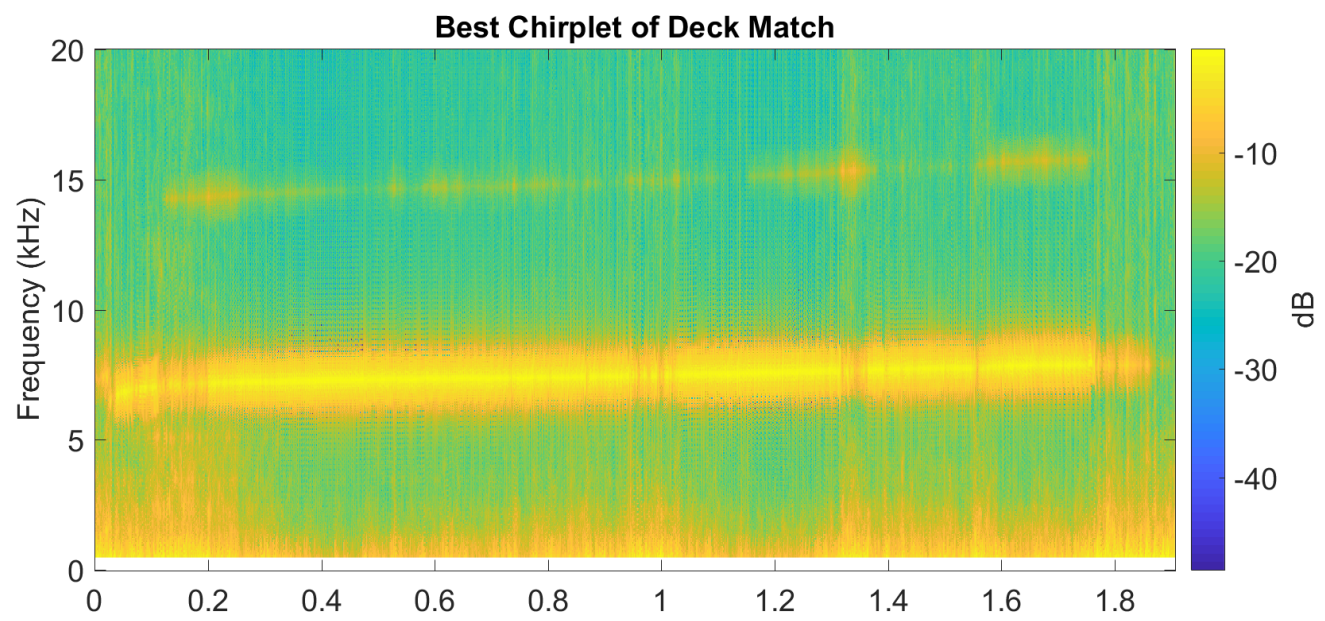

(s)

Figure 5.11: All chirp match results against a Marmoset phee call. Chirplet deck parameters are shown in Table 5.3. The spectrogram window used was: Balanced: .005 Seconds. 


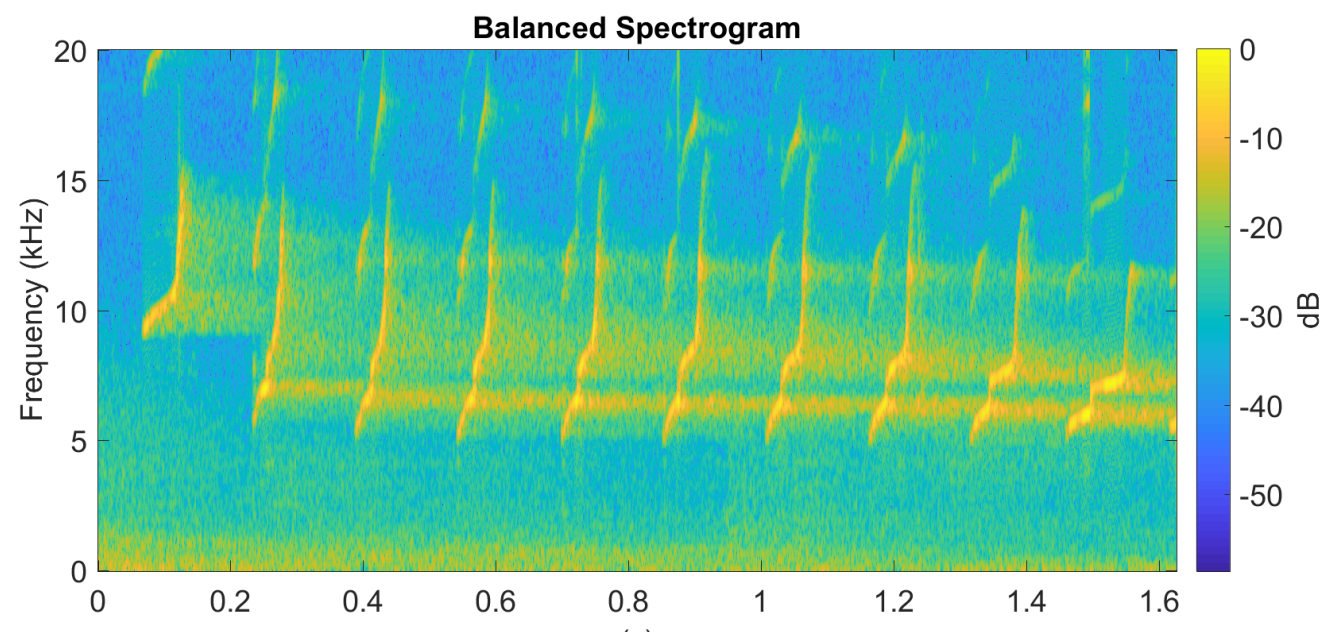

(s)

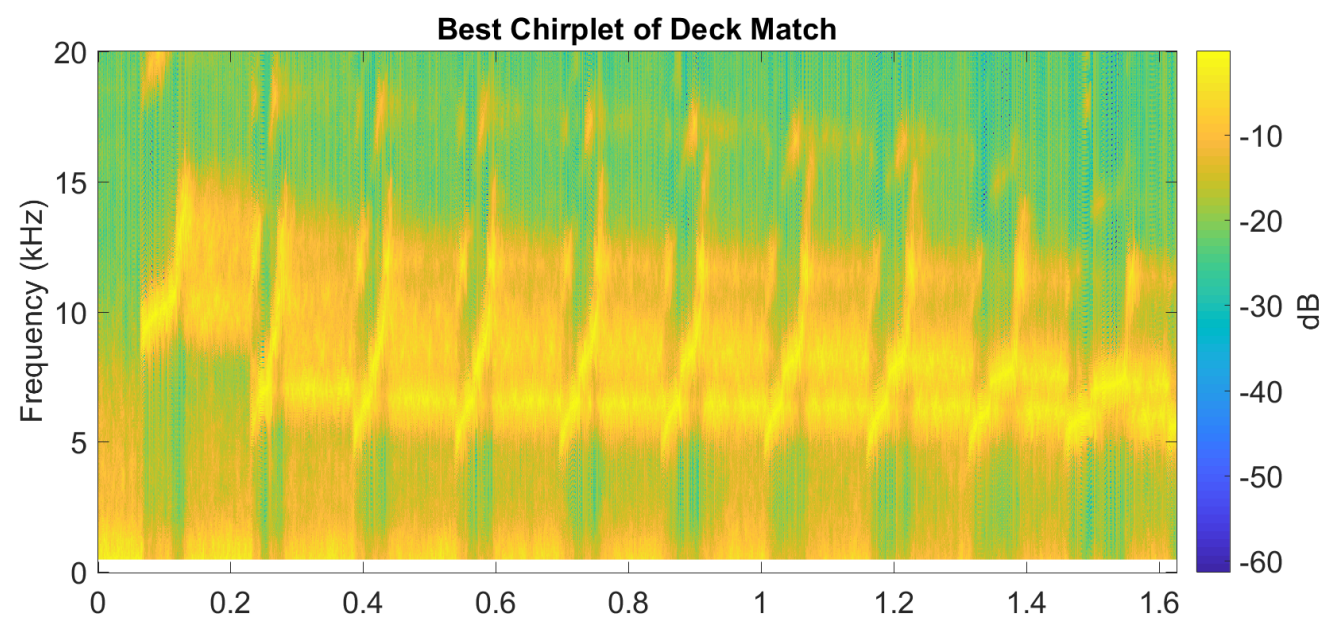

(s)

Figure 5.12: All chirp match results against a Marmoset twitter call. Chirplet deck parameters are shown in Table 5.3. The spectrogram window used was: Balanced: .005 Seconds

\section{$\underline{\text { Sparse Encoding }}$}

At each point in the time-frequency plane a total of $\mathrm{N}$ chirplet matches are examined. In the previous section, we increased chirplet deck size from 14 to 38 
and saw better matches of our signal. This means that for each point in the timefrequency plane, we have outputs from 38 different chirplets. This number could easily grow. How can we keep the information presented to a learning back end relevant? The great number of outputs from chirps can be decreased by selecting best matches or matches which meet a particular threshold. The project will use a max operator to emulate lateral inhibition. The biological inspiration of lateral inhibition was discussed previously. In fact, the max operator was already demonstrated in Figure 5.6 when the best chirp for each time-frequency point was displayed. For this dissertation, a max operator is applied to each spatial point in the time-frequency plane to select for the chirplet that best matches the signal at that point. All other chirplet outputs at that point in time and frequency are zero'ed and only the best chirplet is allowed to pass.

Next a different visualization of chirplet output is demonstrated for illustration purposes. Previously in Figure 5.12, the max operator was used for every timefrequency point. The next result is best chirplet in time. For a given time slice, a max operator is used to pick the very best chirp response occuring at that point in time across all frequencies. Thus for all decks evaluated at a paticuluar point in time, the best chirplet from all the decks and frequencies is displayed. In this way, we find the chirp which matches the signal under study the very best at a certain point in time across all frequency points and across all chirplet types. This analysis uses 7 primary chirp types in two lengths against the Marmoset twitter call. The following results attempt to illustrate the idea that out of our chirp array, best chirp types can be picked. Particular chirps are very best matches to the signal at a point in time. These graphs are simply showing that in a particular time slice, certain chirps located near the signal in frequency and shape are the strongest match. Further, specific chirps of the deck are better fits to the signal as the signal changes over time. 
Also of note, that chirplets of different lengths preferentially match the signal. In Figure 5.13 we see that the shorter chirps are matching more parts of the signal more often due to the nature of the signal changing quickly as a function of time.

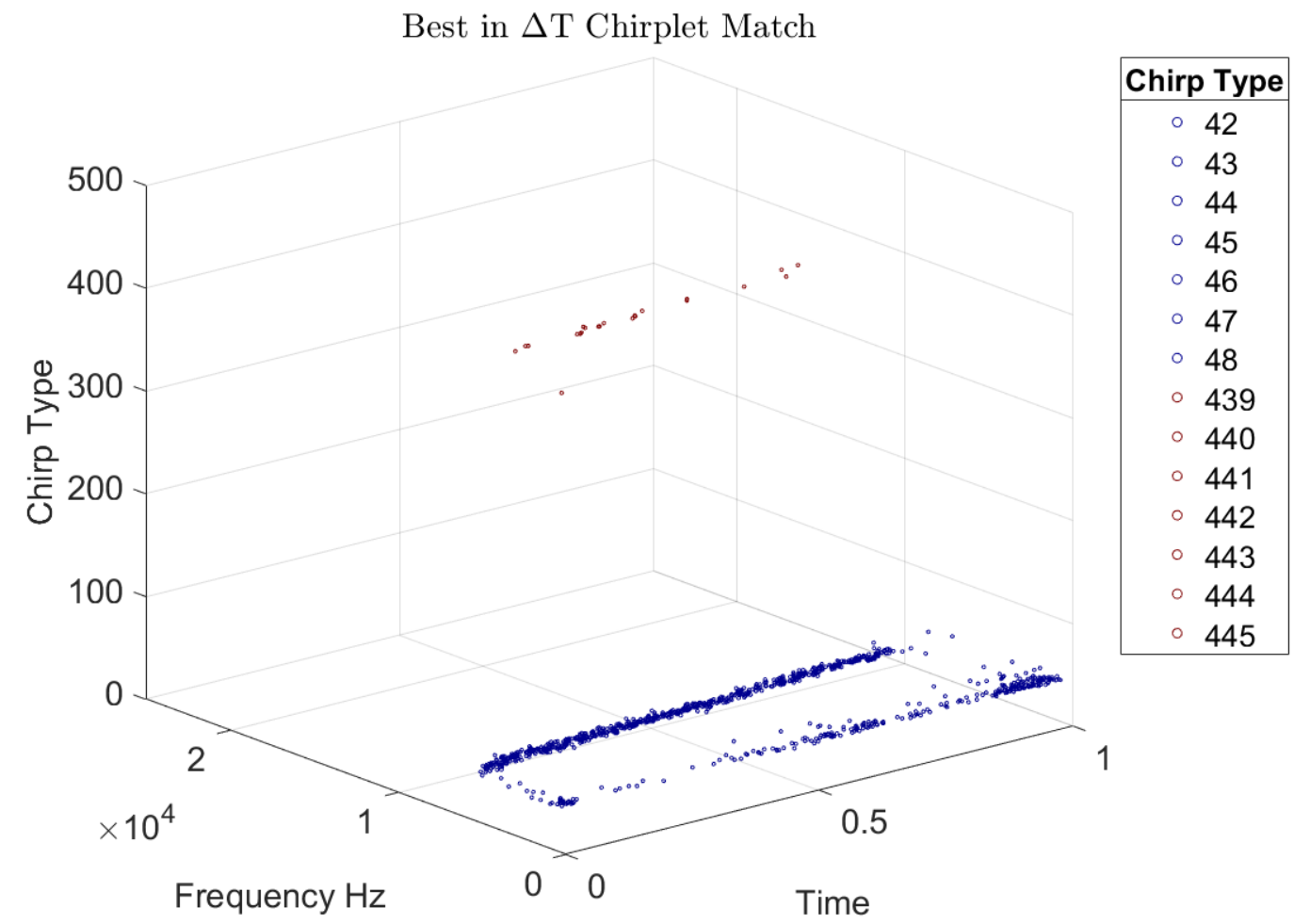

Figure 5.13: Best chirp match to signal across all frequencies and chirp decks for an instant in time. We see the longer chirps represented by the 400 indicators matching the signal less often.

Let us rerun the analysis with only the shorter chirps to better see the matches among these chirp types. Figure 5.14 shows the best chirp match against the trill call for each time window. Figure 5.15 is the same result viewed on the time-frequency axis. 


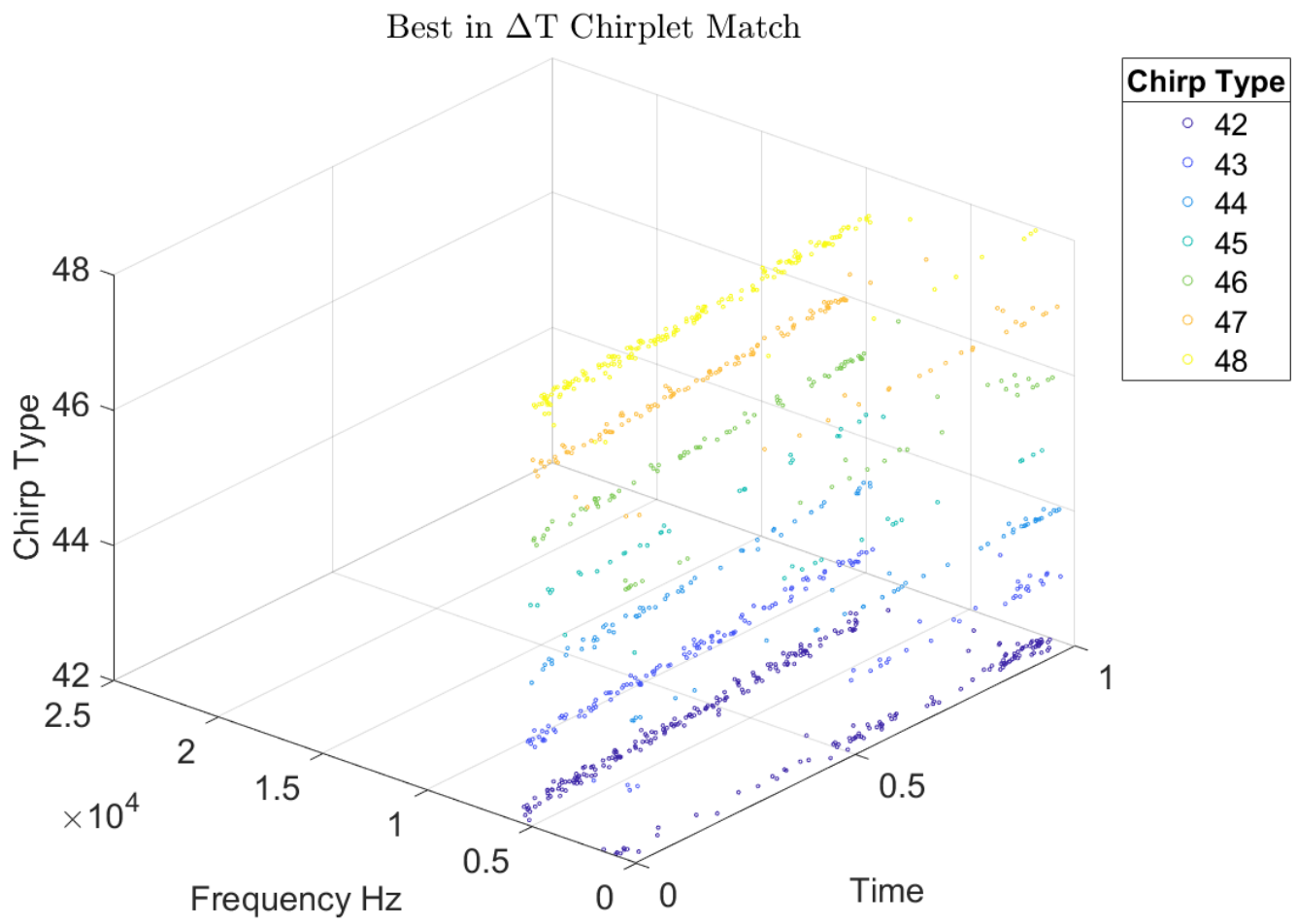

Figure 5.14: Best chirp in window separation. 


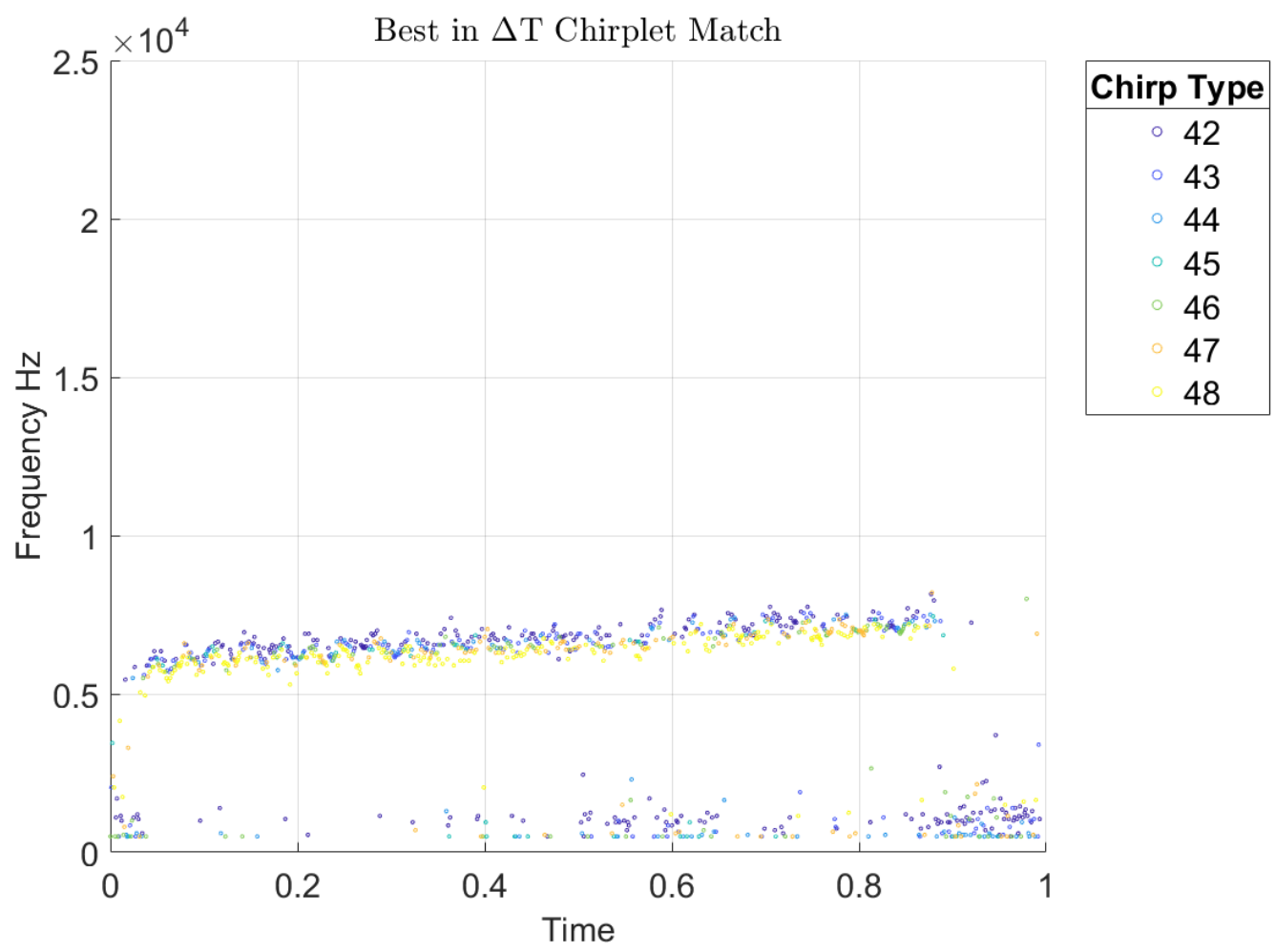

Figure 5.15: Best chirp in window separation.

The figures show that particular chirps are best matches to the signal in time. We also find that the trill call is best fit by any of the seven chirplet types at any particular point in time. This again can be scaled up for many types of chirps. The fact that the trill call is distinctly made up of best chirps, allows us to pass information about specific locations and events in the time-frequency plane to a back end machine learning algorithm.

Another illustration was developed to better show the amount of information which is being conveyed by the front end. A graphic which indicates which type of chirplet is best matching the signal at a particular point in time and frequency was made. This illustration keeps track of which chirplet type is best matching the signal at a particular point in time and frequency and displays that chirplet type via 
color code. Thus the subplot shows not amount of match, but which type of chirplet matches best via color code. For example purposes, let us re-examine the very low foveation demo example, except this time display a chirp identification plot. A graphic displays the relevant types of chirps which are existing and matching for each point in the time-frequency plane. For the chirp identification plot, I first identify which chirplet is matching best at a particular point. I set a threshold of .1 on the output of the matching function for display. Otherwise, unmeaningful best matches clutter the chirp identification plot. 

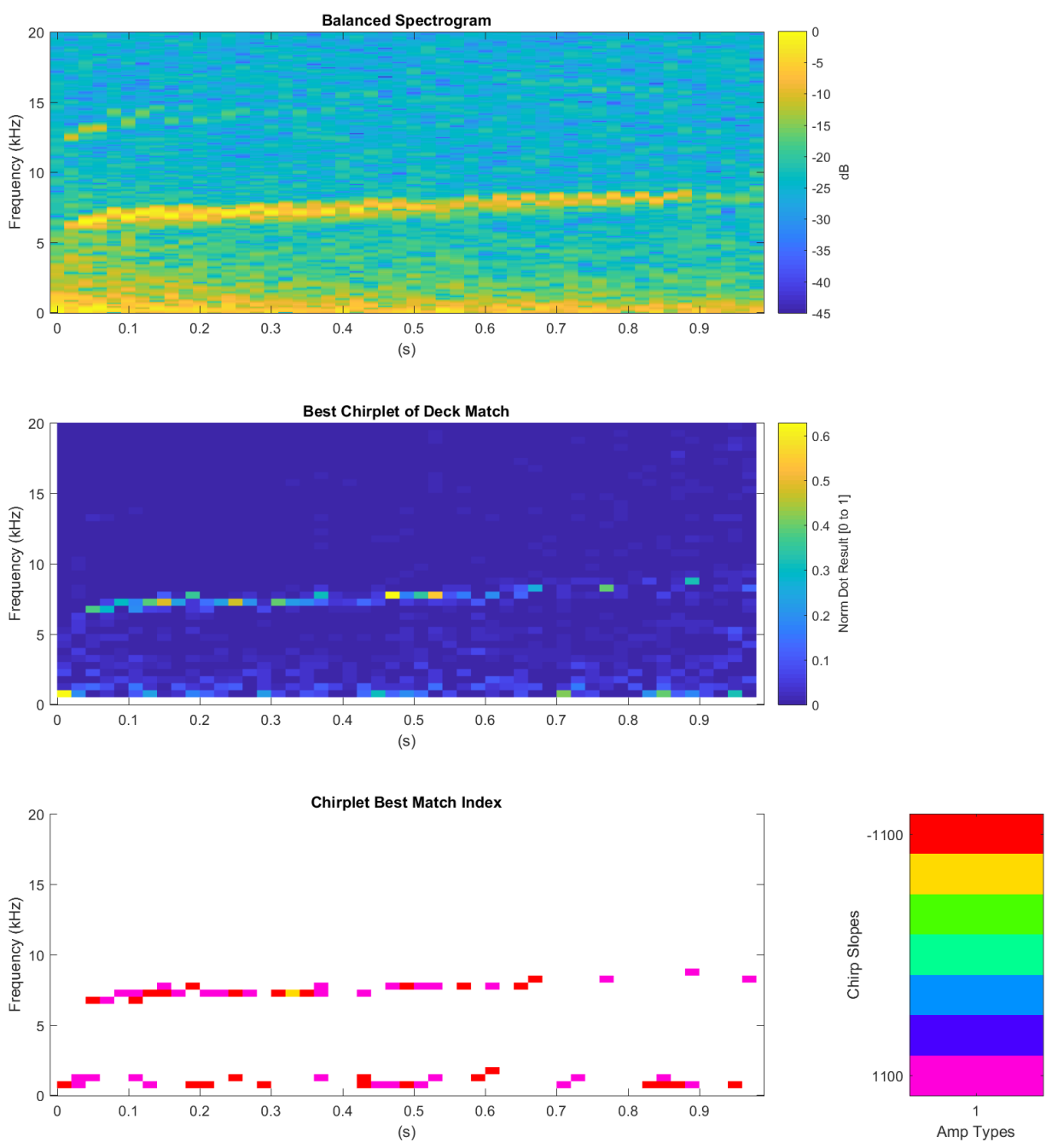

Figure 5.16: Best chirplet match identifcation subplot. The bottom subplot shows which type of chirp is best matching the signal at that particular point in time and frequency. An inlay graphic shows the corresponding color code of chirplet types. Chirplet deck parameters are shown in Table 5.1. The spectrogram window sizes used. Narrowband: .1 Seconds. Balanced: .005 Seconds. Wideband: .0005 Seconds.

Let use re-examine the trill call but with a high spatial and high deck foveation. In this instance there are many more chirplet types (38). The illustration of chirp types becomes more busy visually but we can illustrate the color coding nontheless. 
We can zoom in on the chirplet identification across the plots to see the level of detail which the chirplet processing is affording us as compared to the spectrogram. Again, we are seeing good matching against the signal meaning our chirplet deck is parameterized well. The chirplet identification plot is shown in Figure 5.17. A corresponding zoom is shown in Figure 5.18. It should be noted in Figure 5.17 that the sparse encoding using lateral inhibition is occuring only over deck output. Another component of lateral inhibition is spatial, which is not being implemented in this example. 

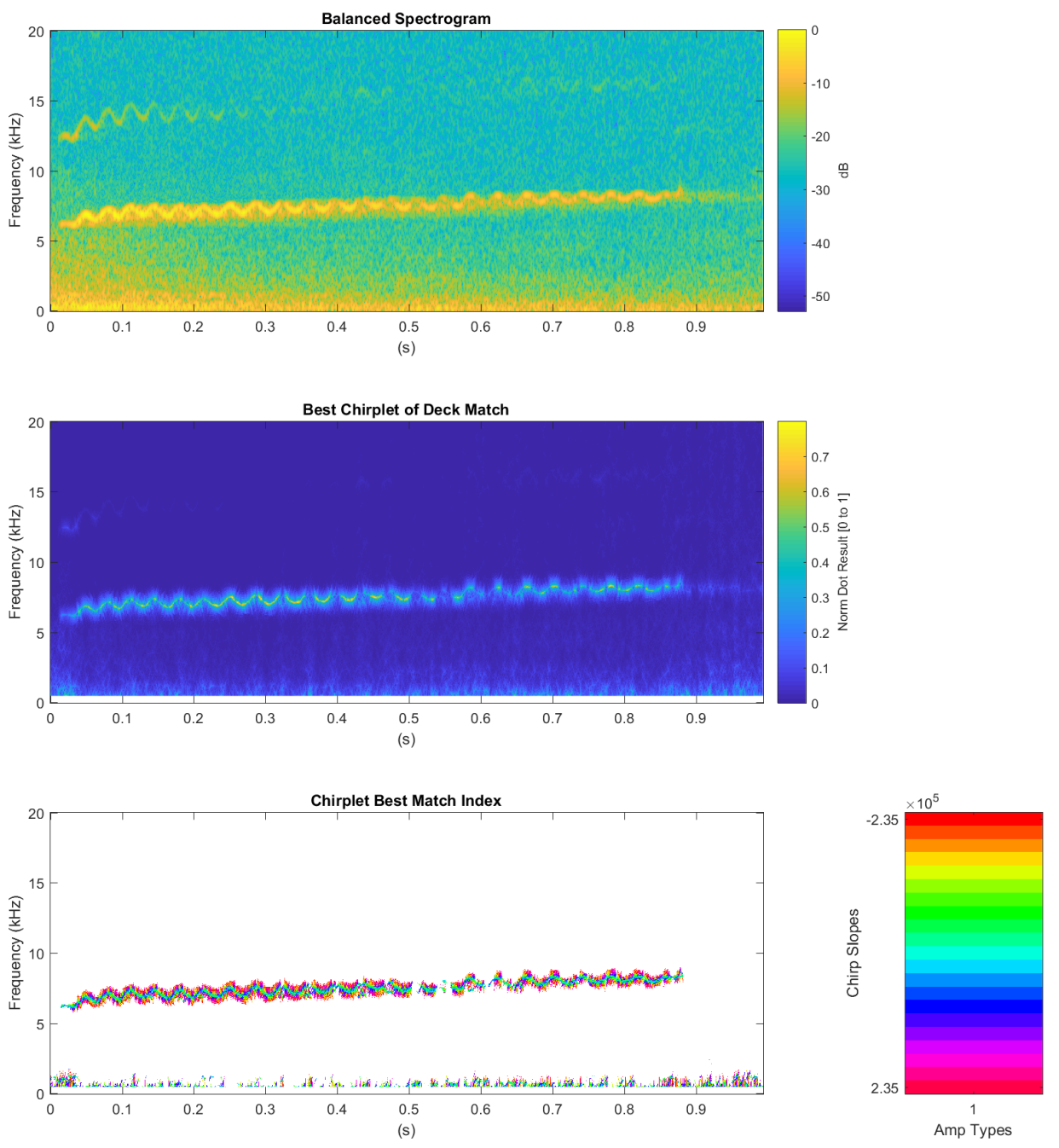

Figure 5.17: Best chirplet match identifcation subplot. The bottom subplot shows which type of chirp (out of 38 types) is best matching the signal at that particular point in time and frequency. An inlay graphic shows the corresponding color coding of chirplet types. Chirplet deck parameters are shown in Table 5.3. The spectrogram window sizes used. Narrowband: .1 Seconds. Balanced: .005 Seconds. Wideband: .0005 Seconds 

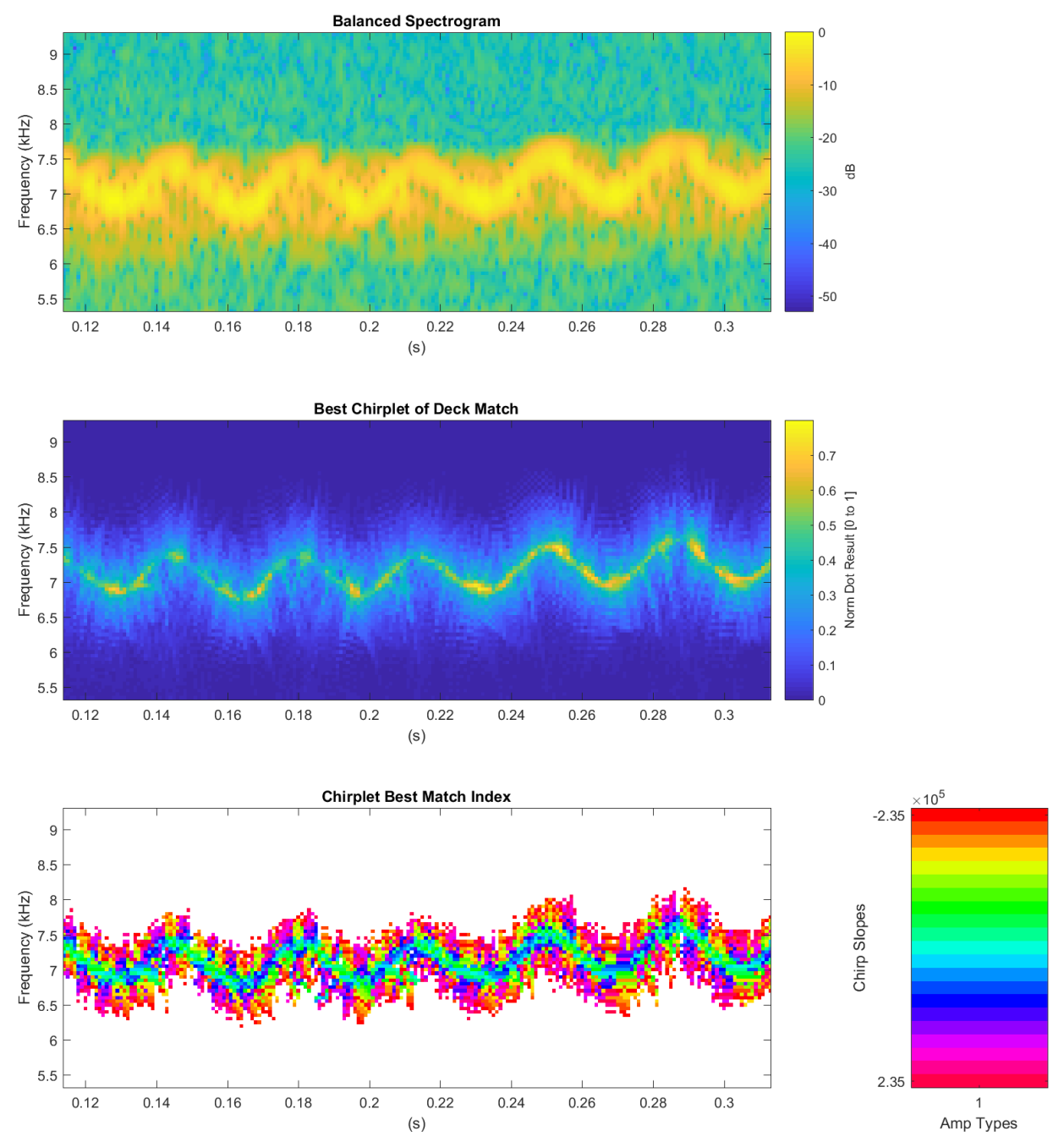

Figure 5.18: Best chirplet match identifcation subplot. The bottom subplot shows which type of chirp (out of 38 types) is best matching the signal at that particular point in time and frequency. An inlay graphic shows the corresponding color coding of chirplet types. Chirplet deck parameters are shown in Table 5.3. The spectrogram window sizes used. Narrowband: .1 Seconds. Balanced: .005 Seconds. Wideband: .0005 Seconds 


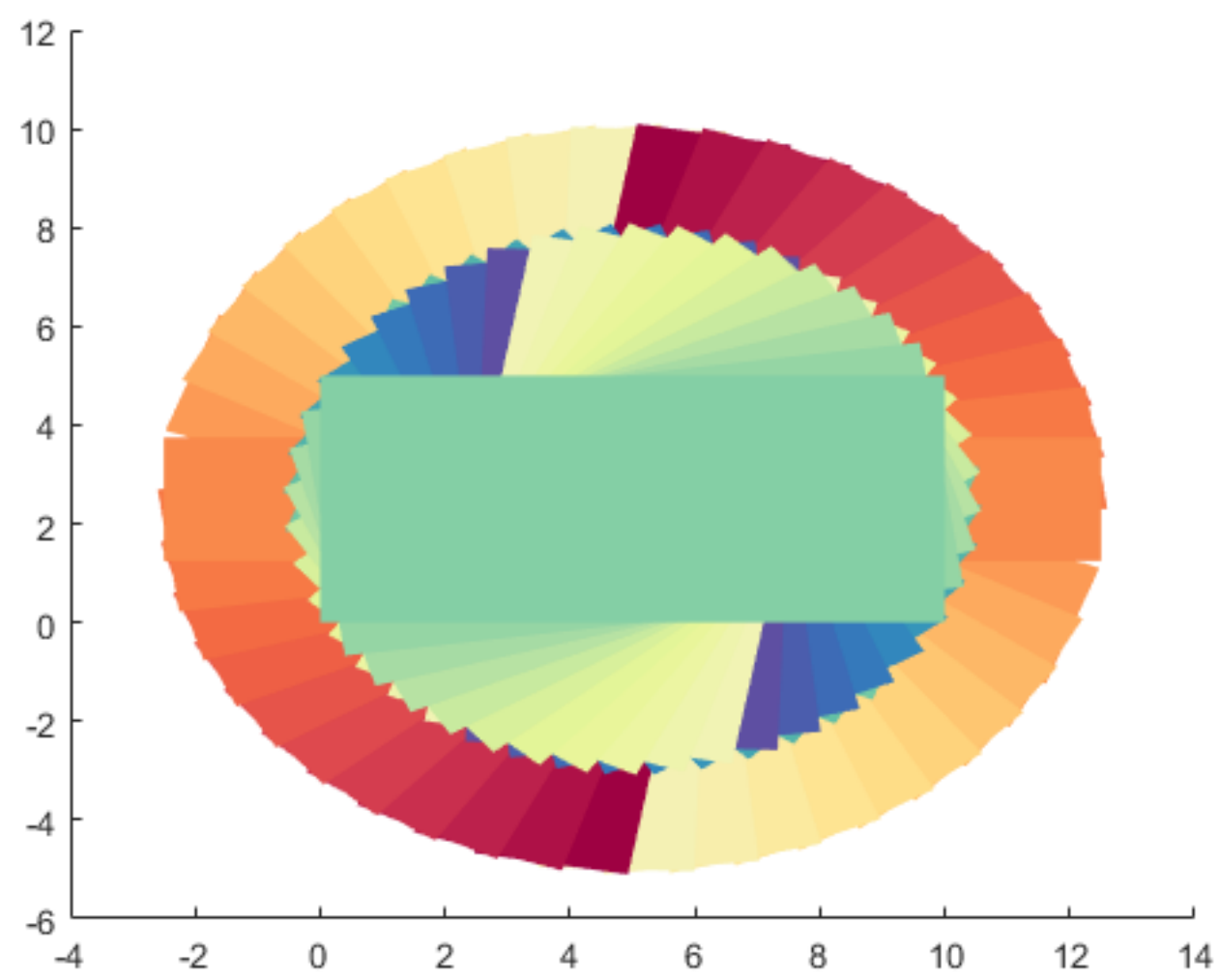

Figure 5.19: Illustration of an experimental visualization inlay graphic. This is an example of the number of sloping chirplets each with different color code for identification plots.

The onset and offset amplitude modulations were introduced into the project to examine if we could detect onset and offset information. Inspiration from biology has shown neurons which distinctly detect the precise onset and offset of incoming signals [20]. We modify the deck parameter set to begin to identify onset and offset markings contained in a signal. Onset and offset modulations were added to the base Gaussian modulation types. This is demonstrated in Figure 5.20. In the figure we see the onset type of chirplet becoming activated before the offset type. We examine onset offset patterns by restricting our plotting methods. 


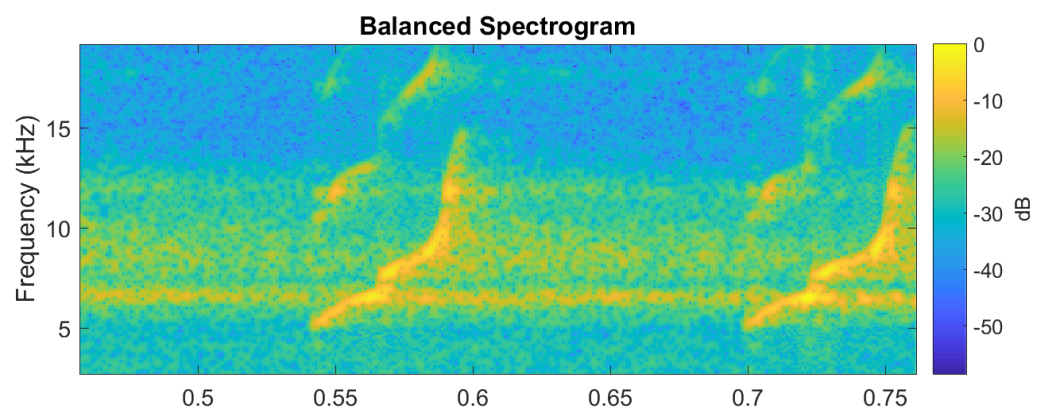

(s)

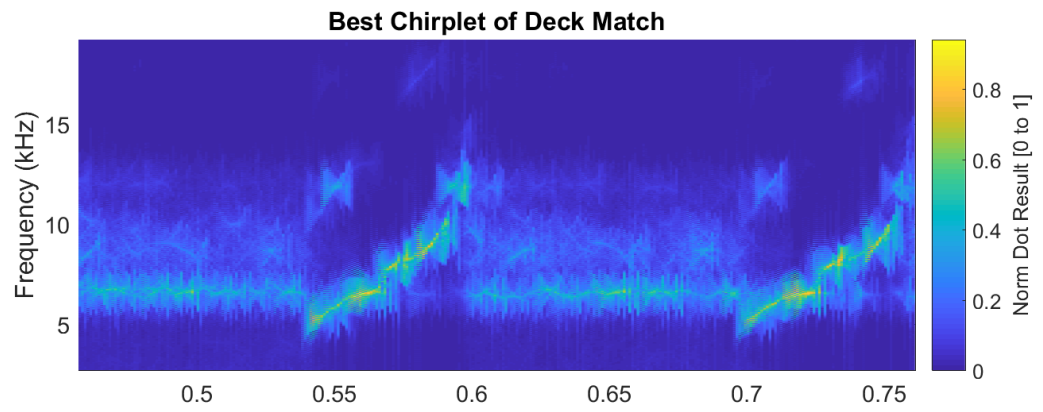

(s)

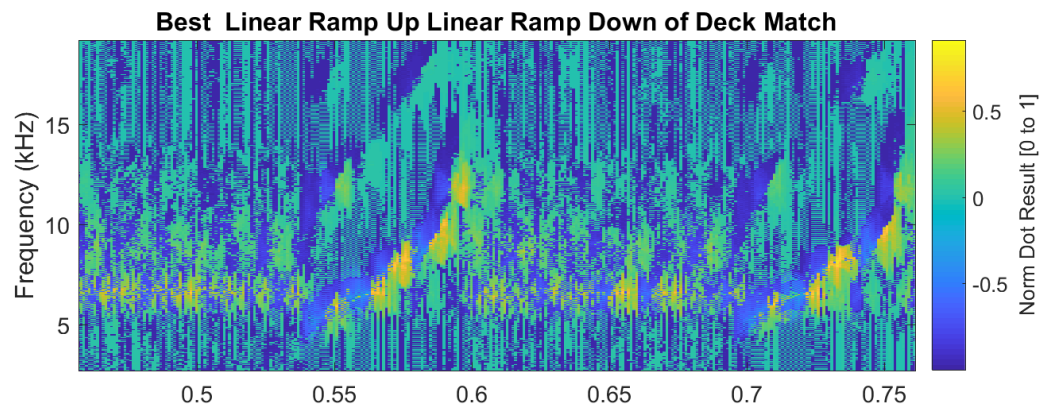

(s)

Figure 5.20: This figure shows a zoomed in version of the marmoset twitter call. We are looking at a particular phrase. The blue color of the bottom figure represents onset type chirplets. The yellow color represents offset type chirplets. We can see that much more onset types are being activated at the onset of a phrase. This is illustrative and what is expected.

As the project is described as a customizeable auditory fovea, an example of customizing the fovea to look at a particular signal is shown next. In this example the band of frequencies around a Marmoset trill are foveated and examined more 
closely. The additional foveation is done with an additional deck parameter option of the front end. In Figure 5.21 we see a lack of additional foveation and in Figure 5.22 we see the foveation around the signal space of interest added. Much more information about the signal can clearly be seen. This information could be relevant for both machine learning as well as illustrate Marmoset call nuances lost with a low foveation or a fixed spectrogram window size. We will now examine the back end to this project and artificial neural networks. 

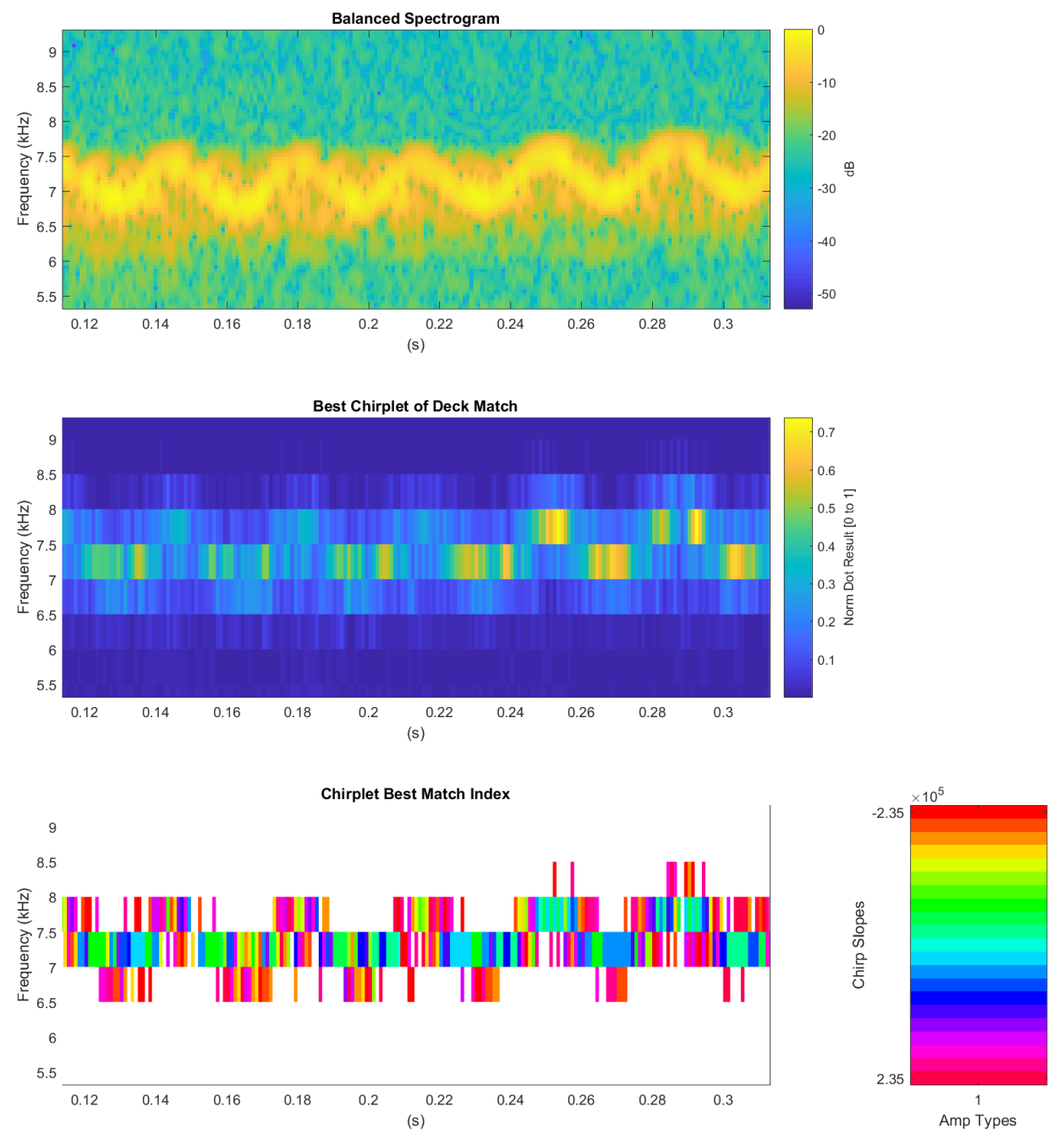

Figure 5.21: A Marmoset trill call wherein the fovea is not customized with higher spatial resolution on a frequency axis band. Chirplet deck parameters are shown in Table 5.3. No additional chirplets are matched in the 6000-8000 $\mathrm{Hz}$ band beyond the default $500 \mathrm{~Hz}$ steppings. 

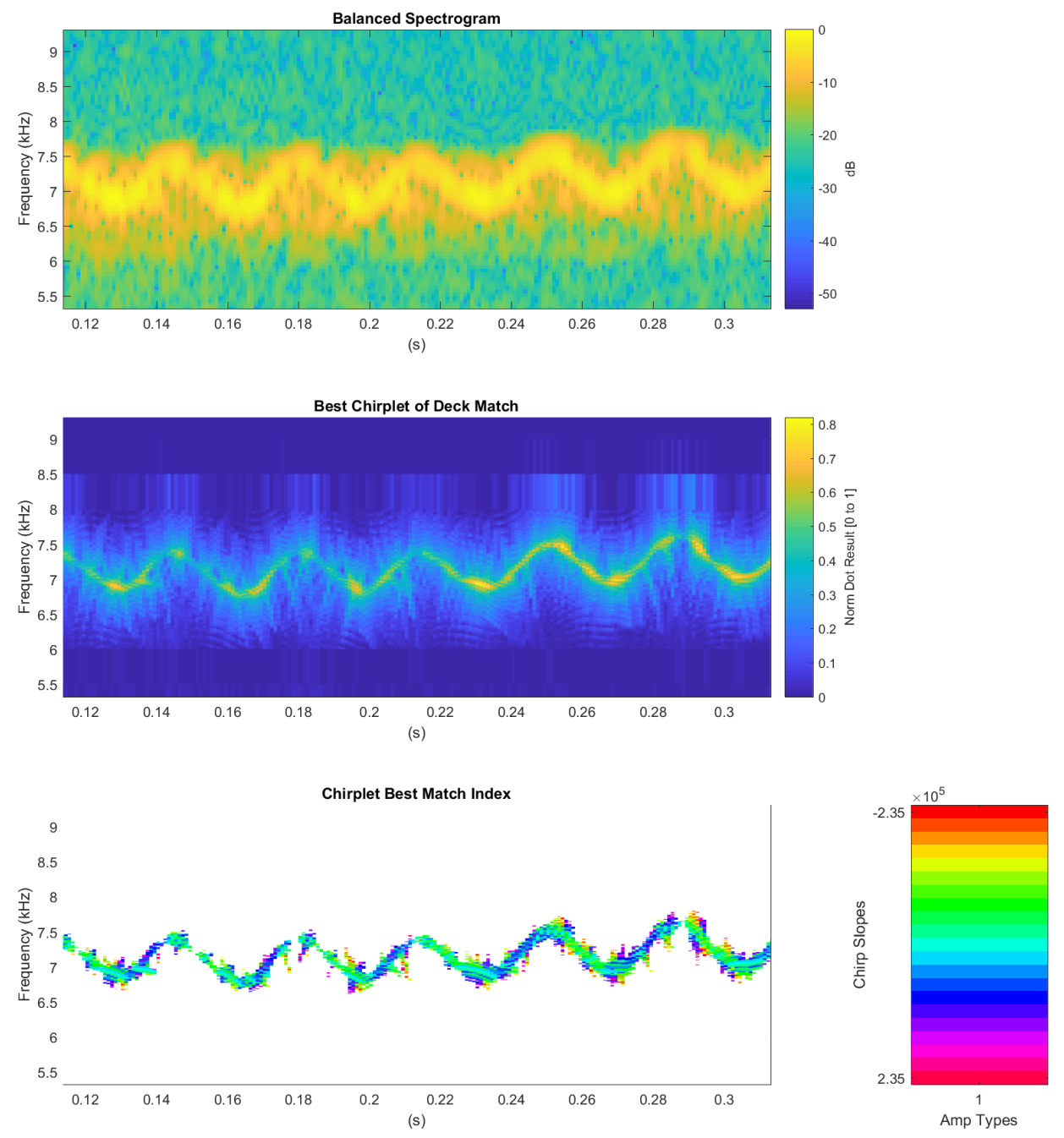

Figure 5.22: A Marmoset trill call wherein the fovea is customized with higher spatial resolution on the frequency axis in a frequency band. Chirplet decks are stamped more tightly along the frequency axis. Chirplet deck parameters are shown in Table 5.3. Additional chirplets are matched in the $6000-8000 \mathrm{~Hz}$ band at $10 \mathrm{~Hz}$ steps. 


\section{ARTIFICIAL NEURAL NETWORKS}

\section{$\underline{\text { Neural Network Background }}$}

This project uses a neural network back end to learn and classify marmoset vocalizations. A neural network is a mathematical algorithm where an input to output mapping is learned. It is a type of machine learning algorithm being used extensively in research and industry for artificial intelligence related applications [43]. Neural networks are used to autonomously drive cars and in speech recognition. The neural network is primarily used for recognition, where without user intervention after a set of data is fed into the network and the network predicts what is in the data.

The algorithm attempts to predict an output from an input through a series of successive weighted functions. These internal functions are termed perceptrons inside the context of neural networks. Information flows from the input of the network, through layers of stacked perceptrons to the output of the network [15]. The output for a neural network designed to classify is a one hot vector which represents a single class. The idea for visual recognition is that a set of pixels representing an image is fed into a neural network. The network which has already learned to classify outputs would output for example that the image contains an apple, orange or banana. These are the defined output classes. In this project, the input to the neural network will be either a spectrogram or a set of chirplet matches and the output of the network ideally will be the marmoset call type which was identified.

The input to the network are termed features in the context of machine learning. The features are a vector of data representative of something which is to be classified. Many different types of features are used in the context of machine learning. Features can range from pixels comprising an image to hand engineered transformations of a particular data source. The neural network in this study is not the topic of research. 
It is used to complete the classification process and used to test the newly developed front end.

Each perceptron of a neural network shares basic similarities. A perceptron or "neuron" which attaches to the input of the network takes input from all input features. A neuron further in the network has connections to all neurons in the previous stage and neurons in the next layer. Each input attachment has a weight associated with it. These weights are parameters of the network which are learned by the network through training. The output of each neuron is passed through a nonlinear function before hitting the next neuron. The nonlinear activation functions were originally inspired by the binary firing of actual neurons. These nonlinearities allow the network to begin to model nonlinear functions [15]. These computations take the form of large matrix multiplies wherein each neuron input is the sum of all previous neuron outputs multiplied by the neuron's weight. The parameters of the network ,or the weights, are changed to make the network learn.

The parameters of the network are used to define a cost function of the network. Reducing this cost function is in essence what happens when the network learns or trains. The basic perceptron is shown in Figure 6.1. Here we see the perceptron taking input from all previous inputs, summing with applied learned weights and passing that sum through the nonlinearity. The output of the nonlinearity becomes this neuron's output into the next stage or neuron. The neural network described are termed fully connected neural networks as each neuron of a layer is connected to every neuron (or feature) before and after it. 


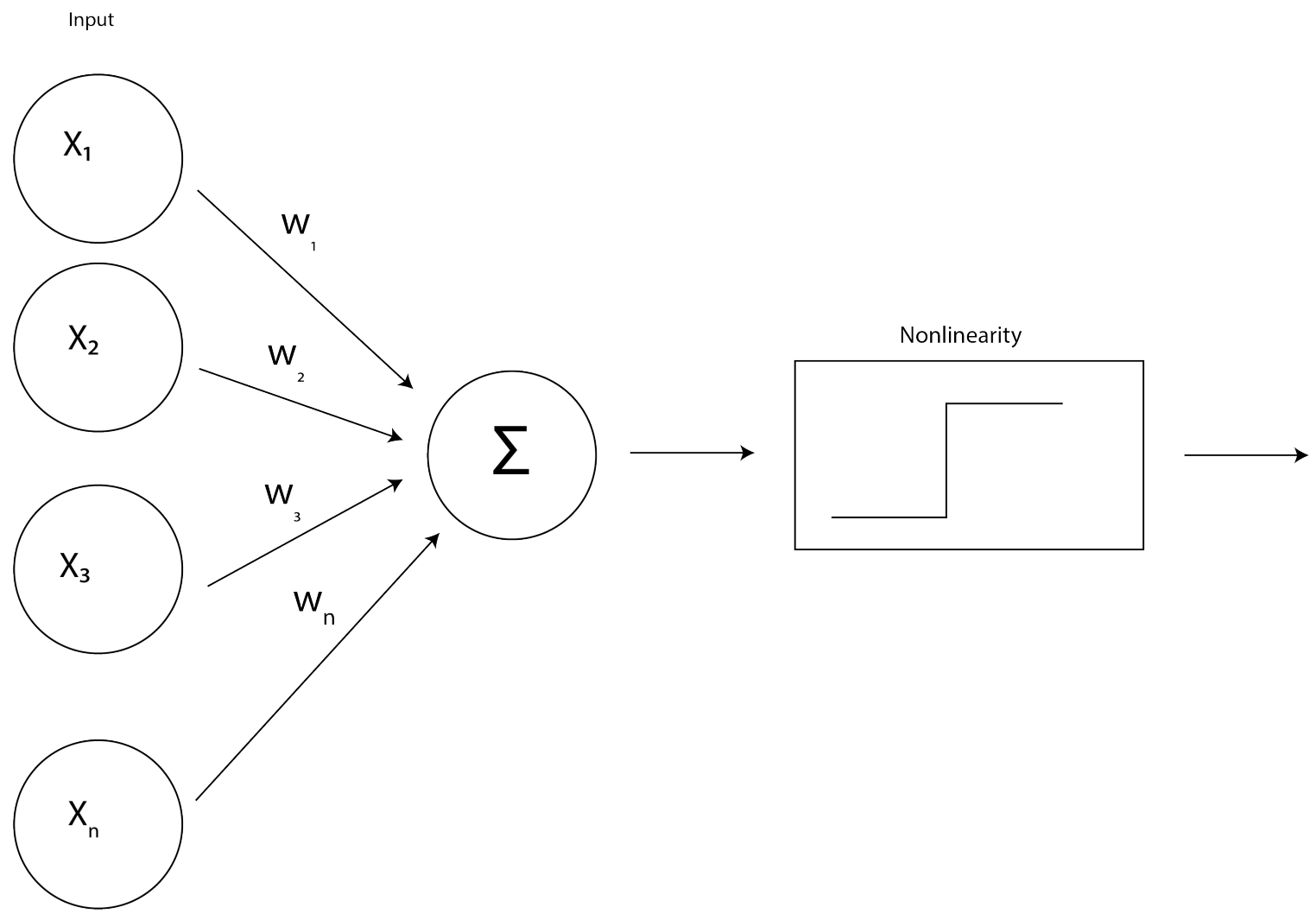

Figure 6.1: The neural network neuron (perceptron).

Neural networks are built up from many individuals neurons. The interconnect of the network begins to look like Figure 6.2. 


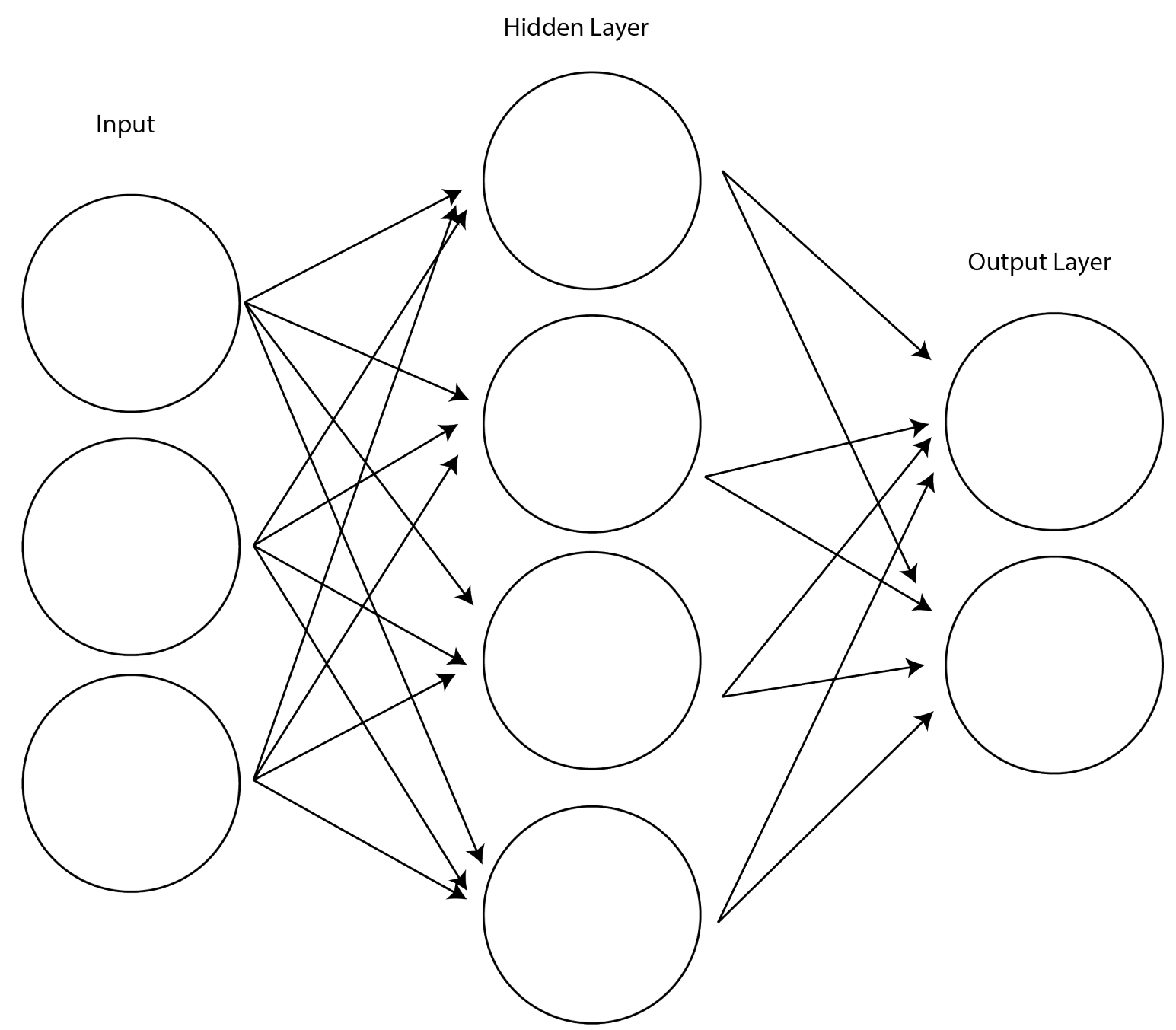

Figure 6.2: Fully connected neural network connections.

A neural network must be trained to learn what it is to classify. The neural network is trained by feeding example data for which the class is known. This set of data is termed the training set. Initially the weights of the network do not work to correctly identify input data. With the training set, the internal functions of the neural network are weighted and re-weighted through training in such a manner to give better classification. A picture of an apple should produce an apple indicator at the output of the neural network. The training of the neural network is done on each successive input of a piece of training data. A function used only in training 
measures the difference between what the output should be, an apple, and the output currently, a banana, which is the total prediction of all the stacked neuron functions. This difference function is termed the cost function and the goal of training a machine learning algorithm is to reduce this cost function. In optimization problems there is an objective function which measures the difference between correct output and current output of a model. The cost function is this objective function. The cost function in total measures the performance of the network in relation to each individual neural network neuron and its weighting. When the cost function is zero, the input is matching the expected output and the inner parameters of the neural network are weighted exactly to produce input to output correctly.

The cost function is itself a function of all internal weights of the neural network. A process termed gradient descent is used to shift all internal weights of the neural network towards the direction which gives the lowest function output and in turn the correct output. A partial derivative of the cost function with respect to each weight inside the entire network is taken. The weights are then adjusted down this gradient, thus reducing the cost function. Each individual weight is shifted in the direction which would decrease the cost function. The network is fed another piece of training data, the cost function is computed, it's partial derivative taken for each weight, the weights are shifted and hopefully the neural network now performs better. This process is done over and over.

Two processes are at work here. Traditional gradient descent requires the full data set to pass through the network before updates to weights are made. Stochastic gradient descent only requires a portion of the data before an update it made. Stochastic gradient descent is what is done in practice as it has been found to effectively converge regardless of a noisier update compared to looking at gradient from the entire data set [43]. It is a learning method and tells us what to do with the 
gradients after they have been calculated. But how do we compute the gradients in the first place given the vast number of parameters?

A method termed backpropogation allows the network to take partial derivatives of all parameters by starting at the output of the network and moving backwards towards the input of the network. Backpropogation is the method which provides the network's gradients. When backpropogation is combined with a learning method such as stochastic gradient descent a neural network is trained. After a neural network is trained using training data, the network is now ready to classify data which has not been previously identified. A network would ideally exactly identify the input data as the appropriate class. However, this is not always the case. To test the accuracy of a neural network, a portion of the training data is set aside to test the neural network. This set becomes a test set. The neural network's accuracy can then be measured on data which the network has never seen before. This is in essence what the network will hopefully achieve, classify unseen data correctly. A percentage of the time, the neural network will classify an input correctly for which we know the correct output. This tells us about the neural network accuracy. It should be noted that machine learning is an incredibly large field and one which is changing every day. The way in which you arrange or construct a neural network is an active area of research. To demonstrate the sheer number of potential arrangements, I cite a graphic which gives an overview of the number of arrangements [41]. This is shown in Figure 6.3. 

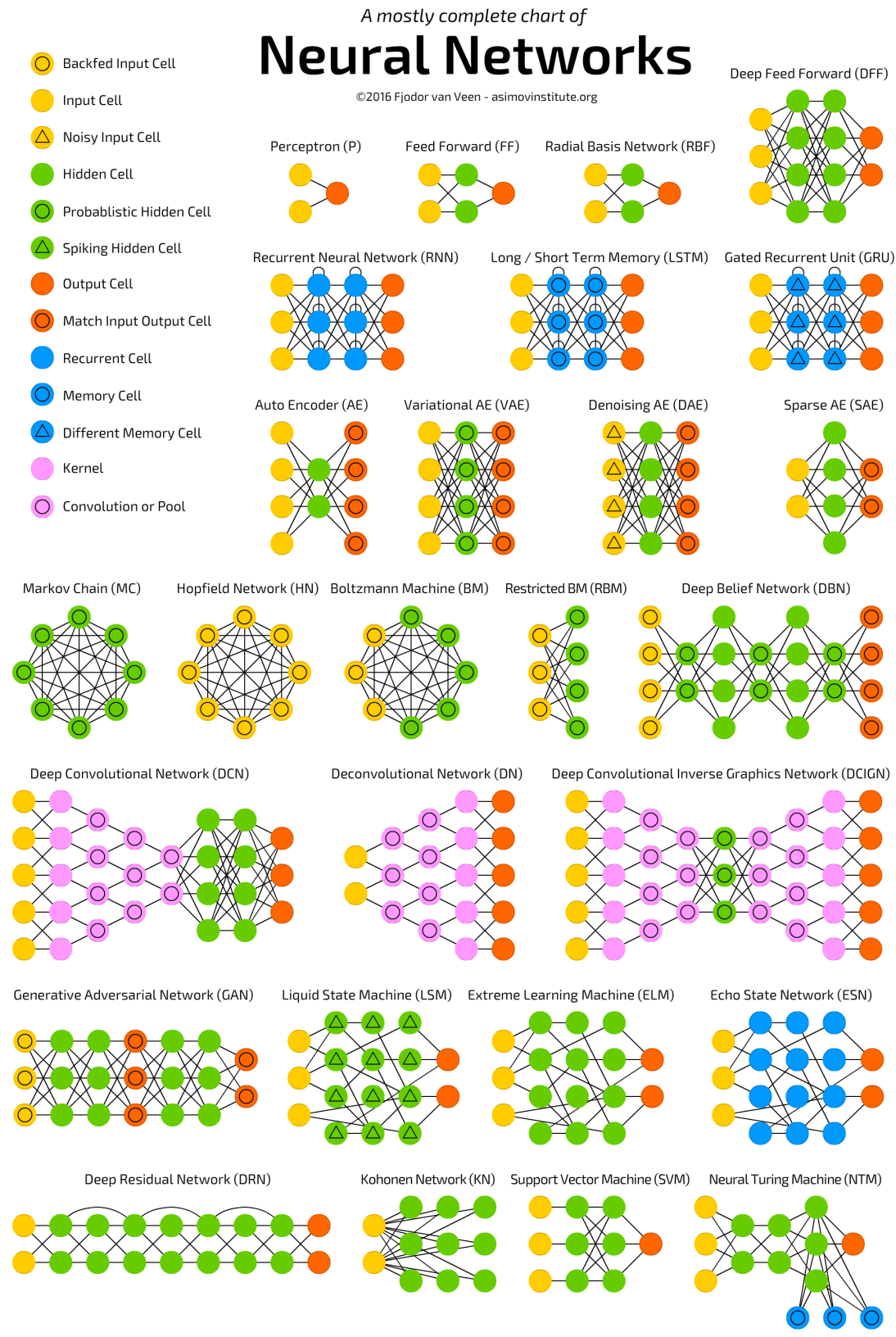

Figure 6.3: Example of machine learning network arrangements [41]. 


\section{Convolutional Neural Networks and TensorFlow}

This project uses a machine learning architecture termed a convolutional neural network. This network differs from a fully connected neural network in that several stages use sparse convolution operations in parts of the network. This is contrasted with a fully connected network where a neuron in the first layer is connected to every input. A more sparse operation at the front end is needed due to the large amount of input features inherant in images or in this case a spectrogram or chirplet scan. Two new operations occur in a convolutional neural network. A convolutional stage occurs wherein a kernel is convolved with the input. In essence, a matrix is slid across the 2-D input. At each step, the kernel weights are multiplied by the input and stored into a new smaller output matrix. A kernel can have many layers wherein each layer contains a different learned set of parameters.

Pooling layers are traditionally inserted between convolution layers in a convolution neural network. Their job is to reduce the number of parameters in the network and reduce complexity. The max pooling operation used in the network is essentially a sliding rectangular max operation. The maximum point in a rectangular window is taken as output.

Convolutional neural networks contain fully connected portions near the end of the pipeline after convolution and pooling has reduced the number of connections from the full input feature set. Fully connected layers contain the traditional perceptrons wherein each neuron is fully connected to all previous inputs and all outputs thereafter.

The output of the Artificial Auditory Fovea was taken into a machine learning framework application programming interface called Tensorflow. Tensorflow was created by Google and has been released as an open source machine learning 
framework $[1,2]$. TensorFlow and an example convolutional classification network was used with some modification to attempt to classify the output of either a spectrogram or Artificial Auditory Fovea match into one of eleven different Marmoset call types. The training and test sets used were taken from an existing paper. This data set included 30 examples of each of 11 types of Marmoset vocalizations [40]. These examples became the input to the overall learning framework. For each example, chirplet processing or a spectrogram was taken of the input sound file. The output of this front end processing was either a 2-D array of magnitude values for the spectrogram or an n-D array of chirplet matches for the chirpletgram. These inputs were then fed to the convolutional network for training.

The paper which provided the data which was also interested in classifying Marmoset calls, chose to use an alternative machine learning library called sci-kit learn. This machine learning library presented issues for several reasons. The paper used a linear predictive coding (LPC) method to extract 64 coefficients representative of a Marmoset sound file to train on. These coefficients were fed to a fully connected neural network. The size of a spectrogram or chirpletgram is many times the size of these LPC vectors and using fully connected neural networks is computationally unattainable. Sci-kit learn does not support GPU training nor does it implement convolutional neural networks [32]. Convolutional networks were explored due to the way in which they subsample incoming images and for their natural use on high resolution image input. The initial convolutional and max pooling layers of the convolutional network reduce the parameters of the network in total to a number which is computationally feasible. Matlab's machine learning toolbox was also explored $[25,26]$. While Matlab does currently have a convolutional network functionality, its input convolution operations only accept images with up to three layers. The input feature image for the Artificial Auditory Fovea scan has N 
layers where each layer represents the matching of a single chirplet across the timefrequency plane. If we have defined 50 different chirplet types in our deck, our input chirplet "image" becomes 50 layers deep. A convolutional network in which an N-D input image could be used was needed. TensorFlow was used as it enables greater customization of machine learning architecture as well as GPU acceleration of network training. Training these convolutional networks on the CPU was found to be many times slower than the training times on a Nvidia Geforce 1070 GTX GPU. The Tensorflow machine learning API was settled on for the back end interface.

\section{Convolutional Network Configuration}

The example convolutional network used had a specific structure to the convolutional network [12]. The layers of the network are as follows: convolution, max pooling, convolution, max pooling, two fully connected layers and a classification layer. The two convolution layers have 32 and 64 filters respectively with kernel sizes of 5 and 3. In Tensorflow, one defines these layers of the network easily using a high level API.

The convolutional network used has hyperparameters which were not modified. Hyperparameters are aspects of the machine learning algorithm which are not explicitly trained (for example the weights in fully connected neural networks) but nonetheless effect how well the network learns. A learning rate of .001, a batch size of 10 and a drop out rate of .25 were used. The learning rate hyperparameter indicates the amount by which a gradient descent is taken. Drop-out is a technique published by Hinton which helps a network generalize by randomly reinitializing a weight of the network as training proceeds [38]. This prevents memorization of the data set or over fitting. Batch size indicates the amount of training examples which go into the stochastic gradient descent method before an update of weights occurs [15]. 
Stochastic gradient descent, unlike strict gradient descent, only uses a portion of the data, indicated by batch size, before the cost function is evaluated, its derivative is taken and parameters are moved in the direction of decreasing the cost function. Like many evolutions of machine learning, stochastic gradient was found to be an effective method when learning large data sets while still allowing generalization and because of this it has become a central method. The convolutional network structure used is illustrated in Fig. 6.4.

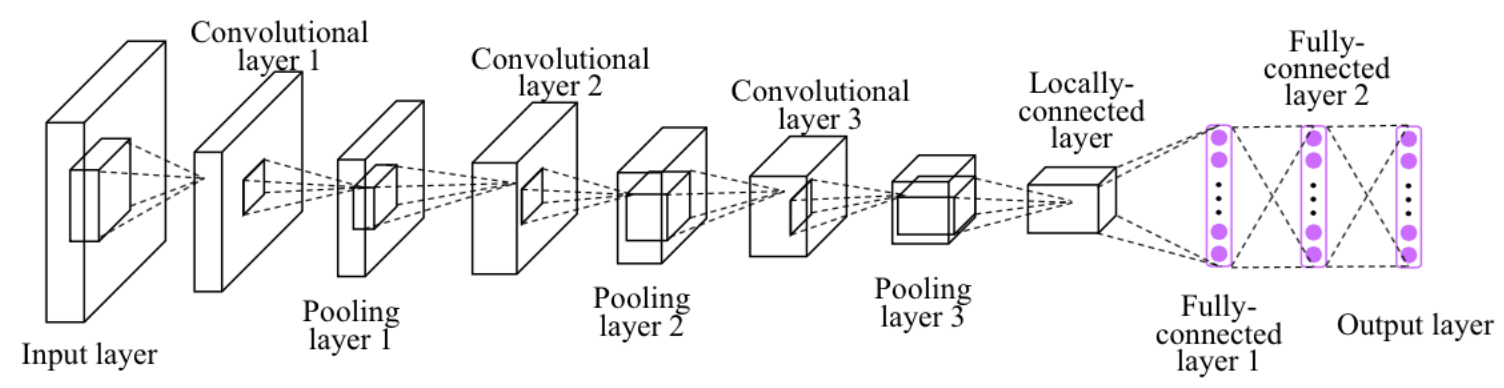

Figure 6.4: Convnet architecture [12].

The training data is fed to the machine learning algorithm many times. One complete presentation of all the training data is termed an epoch. The data is learned via back-propagation and stochastic gradient descent. This learning process is highly abstracted via the Tensorflow API. Nonetheless, we can look at several metrics to compare the ability of the convolutional neural network to learn the data. We can look at the rate at which the cost function is decreased, the ability of the network to learn the training data and the ability of the network to generalize onto data it has not seen before, test data. In machine learning, the ideal goal is for a machine learning network to classify data it has not seen before. It was the hypothesis of this research that given a greater retention of information about the sound source, a more efficient machine learning back end could be realized. This benefit could take 
the form of training time, training accuracy or ability to generalize. The example convolutional network was modified to accept the Artificial Auditory Fovea data.

The convolutional neural network traditionally is structured to accept a fixed sized input. The example Marmoset data was not all identically sized in the time dimension. Thus, each spectrogram or chirpletgram was resized via Matlab to the smallest common dimensions across example data. Matlab's image resize function was used for this preprocessing of the incoming data. This alteration was done identically to chirpletgram data and spectrogram data. This data was then passed from Matlab to Python for use in Tensorflow. Tensorflow, when used with GPU compute, was found to be largely non-deterministic. The training process across trials could not be replicated due to randomness of the training process, especially when using a GPU. I was unable to find a way in which to guarantee all initializations across the network remained identical between runs, given the Tensorflow API. Thus, the examples here are averages across many training attempts to best approximate the ability of the back end identification process. This is the same approach taken by Damien when he trained on the same data using LPC coefficients using Sci-kit Learn [12]. In all instances a sparse operation of the input chirplet data was taken where a max operator is applied across the input data to select the very best chirplet for a particular point in time and frequency.

Elements of data can be exported from Tensorflow as the model is trained. Three pieces of data we can look at are the cost function (or loss), accuracy of the model on the training set and accuracy of the model on unseen data. We examine each as the convolutional network is training. The plots in the next section compare the learning rates of the spectrogram front end versus the Artificial Auditory Fovea front end. We train the network over the data numerous times. We do this again in an attempt to get an average of how the network will train given a set of processed training data. 
We take the average of several training runs for any metric we want to examine. We then look at the cost function and accuracy of the model on the training data as an average of 50 runs. 
101

USING THE CUSTOMIZABLE ARTIFICIAL AUDITORY FOVEA IN MACHINE LEARNING

\section{Chirplet Decks Used for the Front End}

Three different foveations of chirplet scans were chosen for comparison with the convolutional network. The parameters of each are presented in the tables below.

Table 7.1: High deck resolution high spatial resolution.

$\begin{array}{ll}\text { Chirp Lengths } & {[.005 .01] \mathrm{sec}} \\ \text { Begin Slope } & 100 \mathrm{~Hz} / \mathrm{sec} \\ \text { End Slope } & 220000 \mathrm{~Hz} / \mathrm{sec} \\ \text { Slope Step } & 26250 \mathrm{~Hz} / \mathrm{sec} \\ \text { Sampling Interval } & 500 \mathrm{Samples} \\ \text { Frequency Start } & 500 \mathrm{~Hz} \\ \text { Frequency End } & 20000 \mathrm{~Hz} \\ \text { Frequency Step } & 100 \mathrm{~Hz} \\ \text { Total Chirps } & 38\end{array}$


Table 7.2: Low deck resolution high spatial resolution.

$\begin{array}{ll}\text { Chirp Lengths } & {[.01] \mathrm{sec}} \\ \text { Begin Slope } & 100 \mathrm{~Hz} / \mathrm{sec} \\ \text { End Slope } & 1100 \mathrm{~Hz} / \mathrm{sec} \\ \text { Slope Step } & 500 \mathrm{~Hz} / \mathrm{sec} \\ \text { Sampling Interval } & 500 \mathrm{Samples} \\ \text { Frequency Start } & 500 \mathrm{~Hz} \\ \text { Frequency End } & 20000 \mathrm{~Hz} \\ \text { Frequency Step } & 100 \mathrm{~Hz} \\ \text { Total Chirps } & 7\end{array}$

Table 7.3: High deck resolution low spatial resolution.

$\begin{array}{ll}\text { Chirp Lengths } & {[.005 .01] \mathrm{sec}} \\ \text { Begin Slope } & 100 \mathrm{~Hz} / \mathrm{sec} \\ \text { End Slope } & 220000 \mathrm{~Hz} / \mathrm{sec} \\ \text { Slope Step } & 26250 \mathrm{~Hz} / \mathrm{sec} \\ \text { Sampling Interval } & 2000 \mathrm{Samples} \\ \text { Frequency Start } & 500 \mathrm{~Hz} \\ \text { Frequency End } & 20000 \mathrm{~Hz} \\ \text { Frequency Step } & 1000 \mathrm{~Hz} \\ \text { Total Chirps } & 38\end{array}$

The three foveations represent three different resolutions of the Artificial Auditory Fovea. A very low resolution chirplet deck samples the waveform at large strides and contains very few chirplet types (slopes and lengths). A low deck resolution 
represents small amounts of chirp types (length and slope). A low spatial resolution represents a chirplet front end which is sampled grossly along both time and frequency. In other words, a low spatial resolution represents a deck which is stamped at large distances along both the time and frequency axis. A low deck resolution is a chirplet deck which contains few chirplet types in slope and length.

Wideband and narrowband spectrogram methods were tested for comparisons. The FFT size was chosen to be 1024, window length was specified at .005 and .05 seconds respectively and a window overlap of .001 seconds was used. As the wideband spectrogram produces many more data points due to the short windows, it was decided to reduce the input feature set size to match the narrowband input. This was decided after memory issues and training times prohibited using the wideband input unmodified. The modification took the same number of evenly spaced analysis windows from the wideband method as were present in the narrowband method for a given Marmoset call analysis. Thus for each spectrogram analysis on a piece of training data, I calulate the number of points which would have been in a narrowband input and take the same number of evenly spaced anlysis windows from the wideband spectrogram output. The spectrogram method was taken from Mathwork's Matlab [26]. In this way, I produced both narrowband and wideband inputs over all training data.

We will first examine one particular configuration, the narrowband spectrogram input configuration, and watch the accuracy of the convolutional network increase as it trains. Numerous runs of training the network from start are shown in Figure 7.1. 


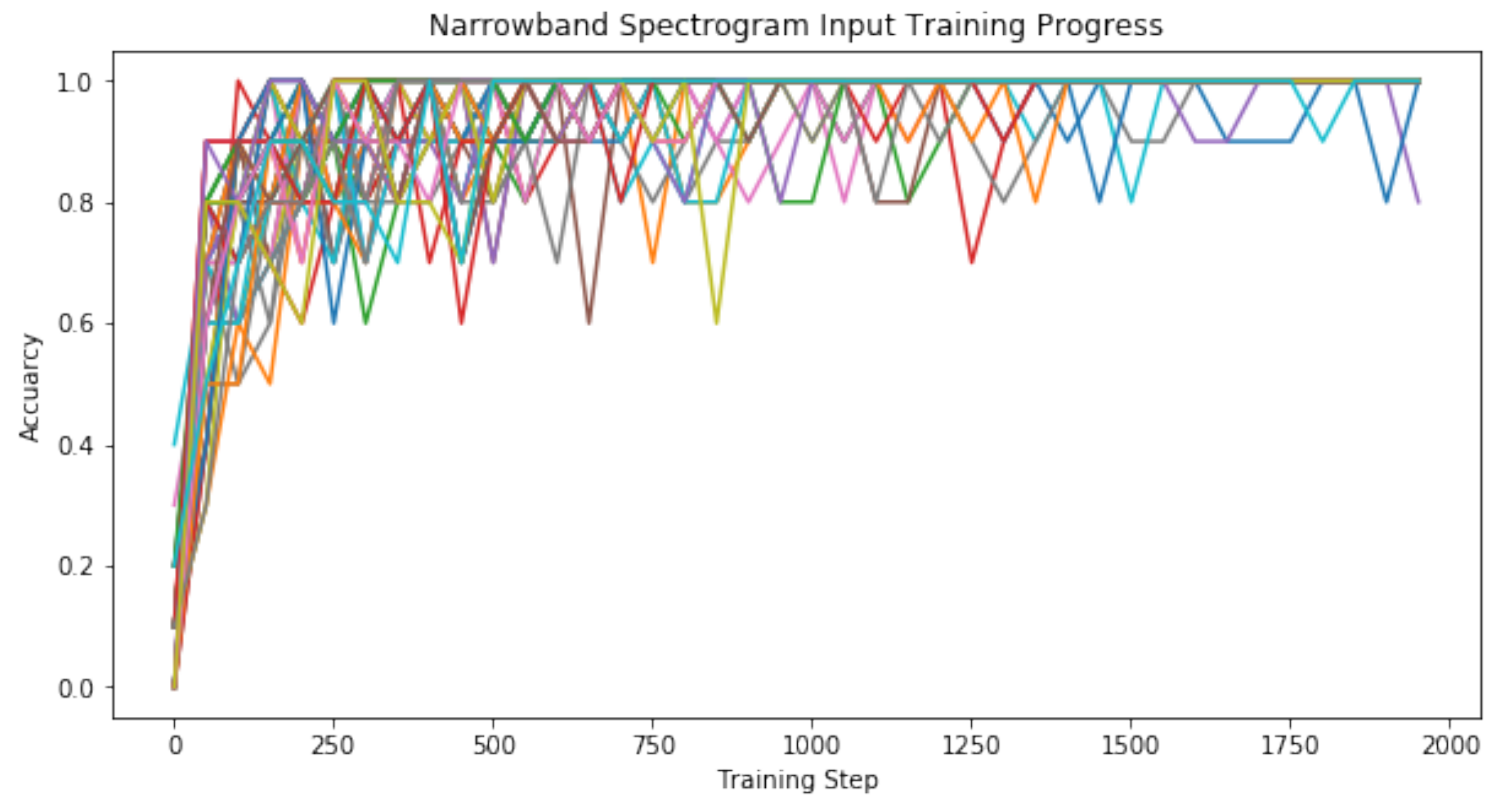

Figure 7.1: Training accuracy of chirplet input network on training set over all independent training runs. Each line represents an independent training of the network from start.

We can also look at the reduction of the cost function over the same number of independent trainings of the network as shown in Figure 7.2. 


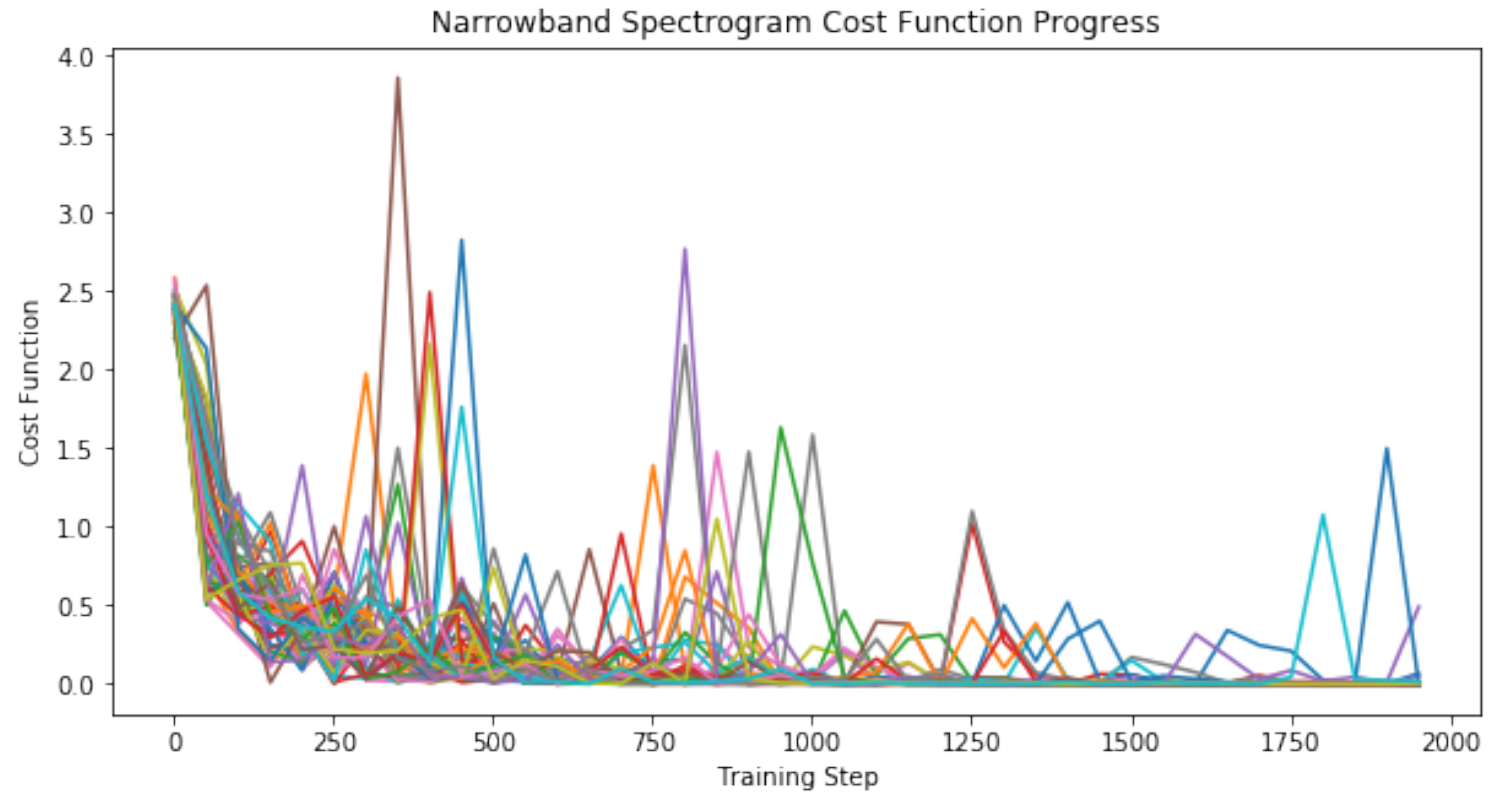

Figure 7.2: Cost function reduction of chirplet input network on training set over all independent training runs. Each line represents an independent training of the network from start.

We can also look at the accuracy of the model on unseen test set data over the same number of independent trainings of the network in Figure 7.3. 


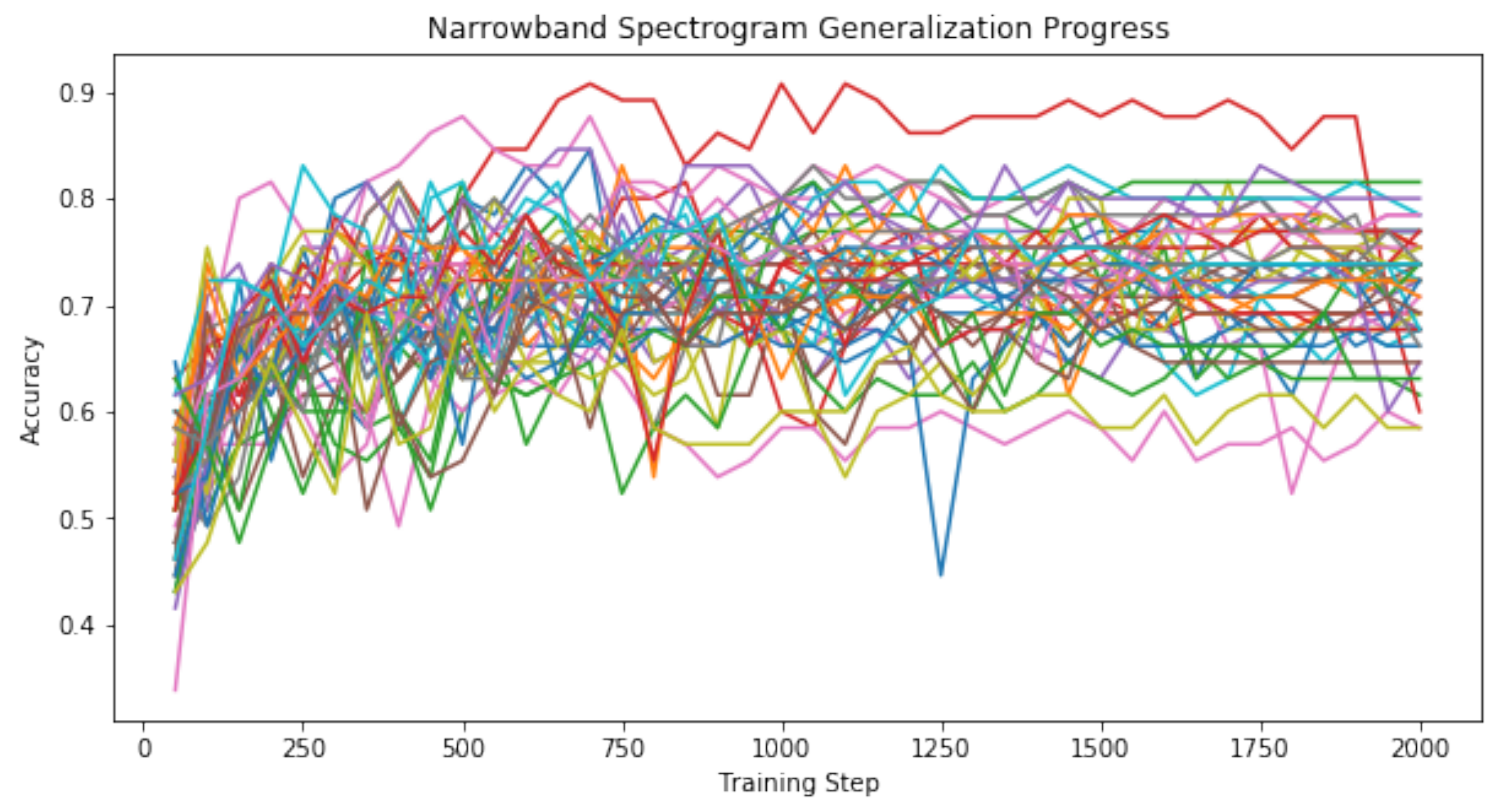

Figure 7.3: Accuracy of chirplet input network on unseen test set data over all independent training runs. Each line represents an independent training of the network from start. Test set accuracy indicates ability of model to generalize.

We now can average the individual runs together to compare the different front ends. One front end is the chirplet processing in different foveations. The other front end is use of the spectrogram in two different windowing lengths. The comparison of the reduction of the cost function among the different front ends is shown in Figure 7.5 and accuracy of the machines over training set data is shown in Figure 7.4. 


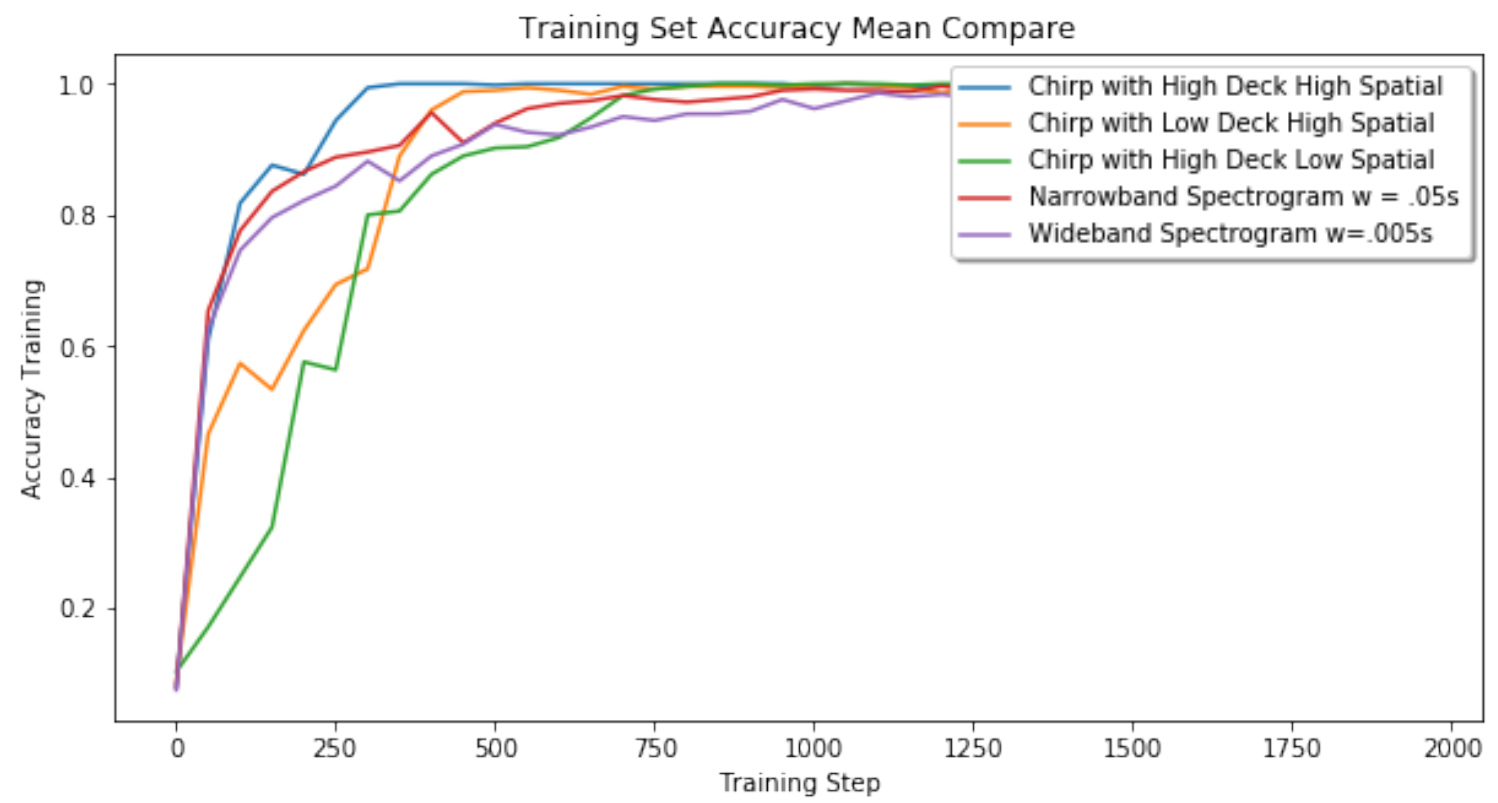

Figure 7.4: Comparison of average training set accuracy across different front ends. Each line is the average of 50 independent trainings of the network on the indicated front end data.

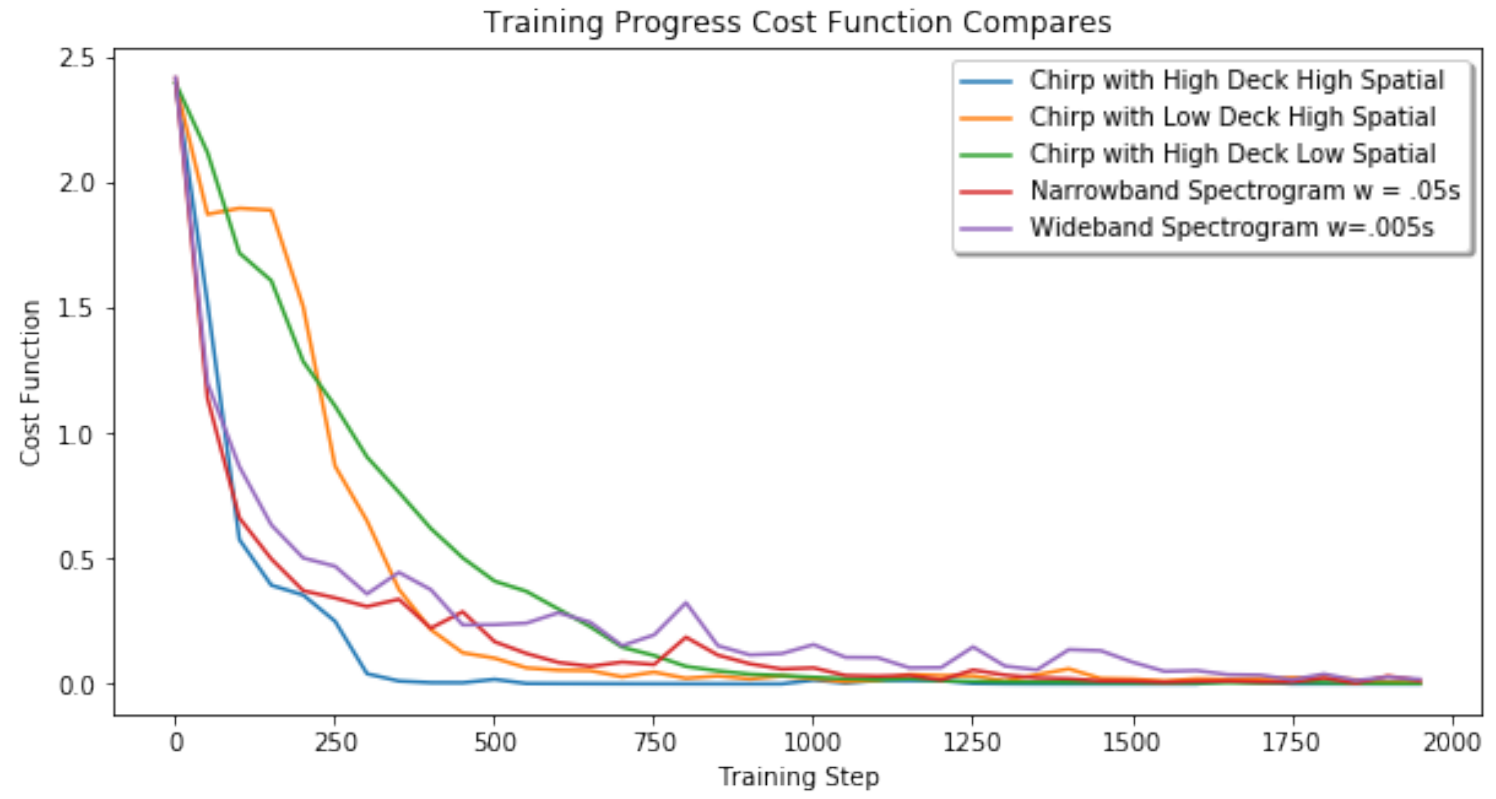

Figure 7.5: Comparison of average cost function reduction across different front ends. Each line is the average of 50 independent trainings of the network on the indicated front end data. 
These results are illustrative. They show the high foveation chirplet front end reaching higher accuracy faster and training faster than the alternative spectrogram methods. This is promising. However, the results also show that the high deck/low spatial and low deck/high spatial pre-processing paradigms performing worse than the spectrogram alone. This suggests that there is a minimum foveation required as compared with the spectrogram.

In machine learning, we hope that the algorithm generalizes to other data it has not seen before. In machine learning, data is typically split into a training set and a test set. The test set is used to test the learned network on unseen data. This can be done with the convolutional network with the spectrogram and chirplet front ends. Again, as the network was found to be nondeterministic, we can average the accuracy of numerous independent trainings of the network. The data was split into 10 percent test data and 90 percent training data randomly between trainings. Tensorflow allows the user to record test set accuracy as the model is training. I found this to be a more illustrative way of looking at generalization of the machine and how it generalizes as the machine trains. The last point in the graphs provided would be the final generalization accuracy of the model after training to the chosen completion step. We can watch the average of 50 runs of complete model training under different chirplet setups. We can compare how the front ends compare in terms of model generalization to unseen data. 


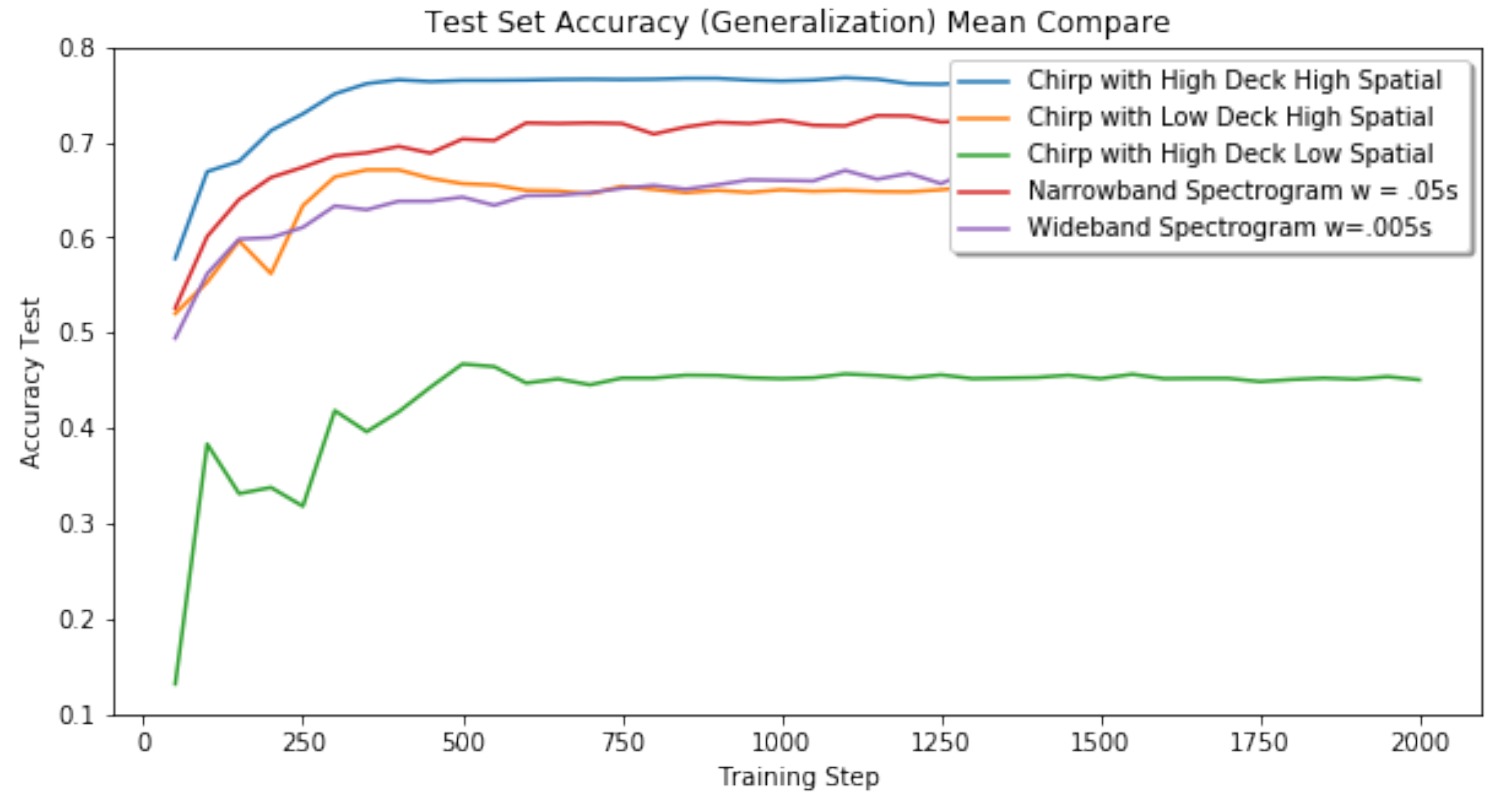

Figure 7.6: Comparison of test set accuracy different front ends. Each line is the average of 50 independent trainings of the network on the indicated front end data.

From this comparison we see that the front end consisting of a chirplet deck with high spatial and high deck foveations is generalizing to the test-set data on average better than any of the other methods. The final points in Figure 7.6 gives us a sense of how this model might generalize to new unseen data sets. The first point in the graphs shows us that the network without training shows very little ability to classify data, training or test set data. This is the purpose of test set accuracy of a machine learning model and ultimately the purpose of neural networks at large, to engage unseen data. The same analysis was run multiple times to verify the repeatability of the testing as another verification in addition to multiple trainings of the model with the same input data. Across multiple complete generations of results, the relative placement of the models remained the same. When we pair the Artificial Auditory Fovea front end with a machine learning back end we train faster and generalize more. 


\section{ARTIFICIAL AUDITORY FOVEA MATLAB CODE}

This section is a guide to show the user how to use the code. The results can be reproduced using the published git respository and the steps of this section [8]. Hopefully this will enable the user to create their own foveas on new data sets to explore classification and identification of new sounds.

The Matlab files in total scan a directory of examplar data and create either chirplet or spectrogram output of each examplar in that directory. Examplar here is one piece of training data and in this case one recording of one Marmoset call. These chirpletgram and spectrogram results for every examplar are then saved out to .mat files which are then read in by the corresponding Python Jupyter Notebook file, Batch_Convo_Poll.ipynb. The Python Notebook file in turn invokes a Python function which loads Tensorflow and trains on the data.

A user should first define a directory structure using set_directories.m

A user should then run Testing_Harness.m. The Matlab testing harness creates

result data sets based on test setups. Test setups are Matlab .mat files which include either a auditory fovea deck parameter structure or a spectrogram windowing indication. The Testing_Harness.m script will for each test setup specified generate the associated test results. These results are either the chirplet data for all exemplar data or the spectrogram data for all exemplar data. These results are saved into Matlab .mat files which are then read in and trained on by the Python/Tensorflow harness.

The machine learning is exectued by running the Python Jupyter Notebook inside an Anaconda Tensorflow Environment. This is done by running the Batch_Convo_Poll.ipynb Python Jupyter notebook. This notebook will for each results file generated, train the convolutional network using that data. 
Results can then be visualized using the extract_tensorboard.ipynb Jupyter notebook. This script parses through all the Tensorflow temporary files to graph the convolutional network's training progress over time. This is how the results section graphs were generated.

To regenerate the machine learning results from the paper one should:

1. Clone the git repository at https://github.com/ccasebeer/A-CustomizableArtificial-Auditory-Fovea.git [8].

2. Edit the Matlab set_directories.m file appropriately and run the script.

3. Move the contents of the provided test_setups directory to the mat_test_dir as defined using set_directories.m.

4. Run Matlab Testing_Harness.m.

5. Run Python Batch_Convo_Poll.ipynb.

6. Run Python Visualize_Tensorboard.ipynb.

The overall system is composed of the following Matlab and Python files:

Matlab Core:

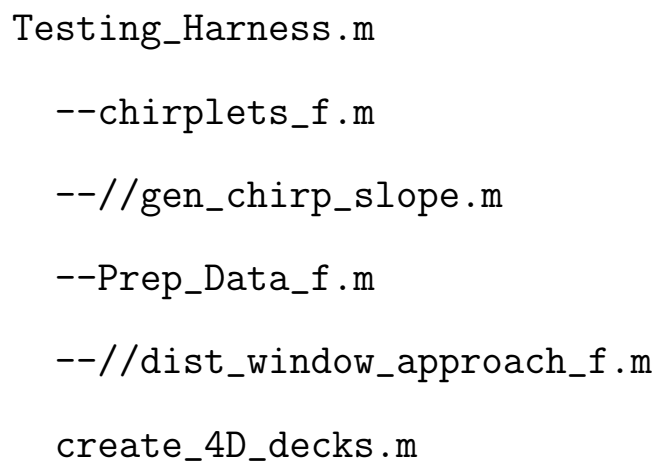




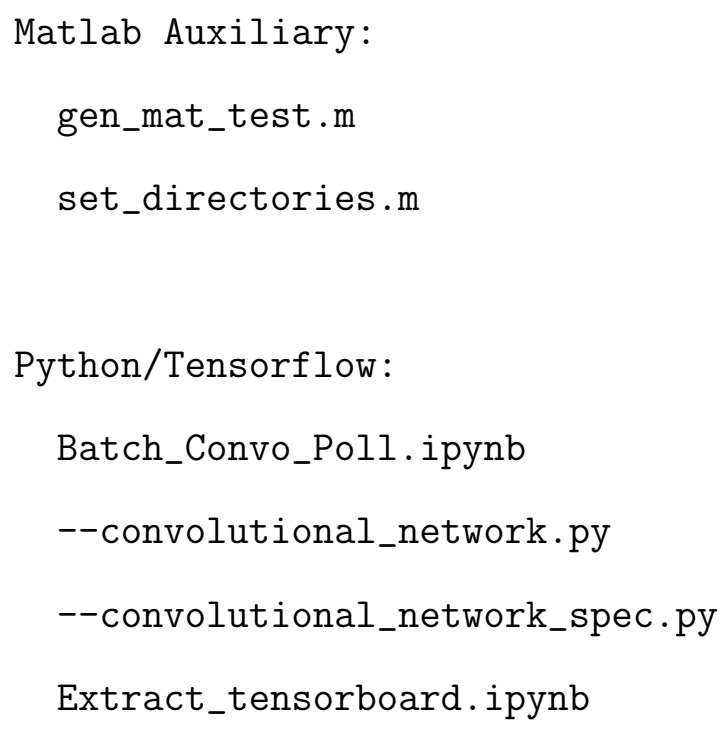

Short descriptions of the code files are as follows:

1. chirplets_f.m - Create all the chirplet time waveforms given the deck parameters.

2. gen_chirp_slope.m - Create a single chirplet given slope and length parameters.

3. Prep_Data_f.m - Recursively works through all exemplar data given a folder structure.

4. dist_window_approach_f.m - Function which compares exemplar against a given chirp.

5. create_4D_decks.m - Restructures the chirplet output into a N-D chirpletgram for use in a convolutional network and in a format that Tensorflow expects.

6. gen_mat_test.m - Creates a test setup Matlab file.

7. Batch_Convo_Poll.ipynb - Excercises either of the convolutional network Tensorflow schemes. 
8. convolutional_network.py - Runs all chirpletgram data through defined convnet.

9. convolutional_network_spec.py - Runs all spectrogram data through defined convnet.

10. Extract_tensorboard.ipynb - Exctracts Tensorflow parameters for visualization.

An example chirplet and spectrogram training setup is provided. This setup specifies different foveations of the chirplet front end and two different spectrogram window setups. These are the same front end configurations as reported in the results section of this dissertation. The following files should live in your directory_setup.mat_test_dir directory (defined when running set_directories.m) before running Testing_Harness.m:

Chirp_High_Deck_High_Spatial_05_18_18.mat

Chirp_High_Deck_Low_Spatial_05_18_18.mat

Chirp_Low_Deck_High_Spatial_05_18_18.mat

Spec_Narrow_05_18_18.mat

Spec_Wide_05_18_18.mat

The following software was used:

Windows 10

Matlab 2017a

Anaconda3-5.0.1 64 bit 'Anaconda3-5.0.1-Windows-x86_64.exe'

$--\backslash \backslash$ Tensorflow 1.5 .0

$--\backslash \backslash$ Tensorboard 1.5 .0

--\\ Jupyter Notebook 
Anaconda is a Python data science environemnt. Tensorflow was installed to this environment. The chosen version of Tensorflow was 1.5.0. The GPU version of Tensorflow was used with a Nvidia 1070 GTX GPU. The CPU version of Tensorflow will take considerably longer to generate the results and train against the data. Jupyter Notebook is an an interactive Python environment which allows in line code visualization and exploration. A Tensorflow environment must be created as per the Tensorflow install instructions and that environment activated before running the Python scripts. 


\section{CONCLUSION}

This dissertation has provided an alternative front end towards audio learning and classification methods. The inspiration has been taken from biology and then framed using engineering of time-frequency analysis. Using the central tenet of timefrequency analysis, an alternative front end was paired with a standard machine learning back end. Initial results show that machine learning can benefit from having a feature extractor which pays attention to biologically relevant information. A high resolution fovea can improve machine learning as compared with standard spectrogram input.

\section{$\underline{\text { Future Directions }}$}

There is fantastic opportunity for future applications of this research. The following areas are ways that can improve the current work. The current brute force matching processing done in the time-domain is computationally inefficient. To alleviate this processing time, several ideas are presented. The first involves an idea presented by Smith [37]. The idea is that chirplets of greatest interest can be found and fit in the frequency domain. This would allow the user to take a Fourier transform of a signal using fast FFT algorithms. The chirplets of interest that should be fit to the signal can then be identified in the Fourier domain. This is allowed as the Fourier transform of the Guassian-windowed chirp is a Guassian pulse in the frequency domain [31]. A complex Gaussian fitting process in the spectral domain has been shown to identify both Gaussian amplitude-envelope and linear chirp rate of the underlying chirp. This process would reduce the computational complexity by not computing chirplets that are not likely to match. A second alternative would be to use the spectrogram to identify regions of the time-frequency domain which 
contain energy. Instead of scanning the entire space including spaces with little or no energy, a user would only process chirps in areas of energy about a threshold. This too would reduce the front end processing workload.

Another metric that is of interest for evaluating machine learning results is the confusion matrix. In a confusion matrix, training and test results of machine learning are shown for each examplar class. A user can see the breakdown of where each example data was classified, either into the correct class or into another of the other incorrect classes. In the training data used, there are a number of sub-types of Marmoset calls which are very similar. The future idea is that since we are retaining fine details associated with the calls inside the chirpletgram, the machine learning algorithm will be able to distinguish between sub-classes with greater accuracy than a fixed spectrogram method. We could examine this behavior by building a confusion matrix for the learning results and manually foveating the differences we observe between subclasses. A well foveated chirplet front end should allow greater distinction between very similar sub-classes. The metric to gauge this would be the confusion matrix.

One proposed method of decreasing the amount of non-relevant information to the back end process is to set a threshold for a given chirp. Only chirplet activity above some given global threshold would be allowed to the back end of the algorithm. This is a method which could improve machine learning output. We can visualize setting a threshold with a number of tools, the histogram and the cumulative distribution fiction. Figure 9.1 shows the match of a particular chirplet type as we match across the Marmoset trill call. This is described by a histogram, where we see the number of times the chirp matches for a given strength of match. Figure 9.2 shows a cumulative distribution function (CDF) for a given chirp's response across the trill call [11].

Using the CDF, a threshold could be set. The cumulative distribution function 
shows us the percent probability that a random variable, $\mathrm{T}$, will take on a value less than $t$ [22]. Only outputs above that threshold could be taken as meaningful and the rest can be set to 0 . We could say that only the top 10th percentile of activations of a chirplet are allowed to pass into the back end. The lower 90th percentile would be set to 0 and interpreted as unmeaningful. This is one way in which information presented to back end algorithms might be kept meaningful. The current method implemented a max operator for each point in the time-frequency plane to select only the best matching chirplet output. A threshold function could be added and examined as part of more pre-processing and its effect on machine learning accuracy, speed and generalization.

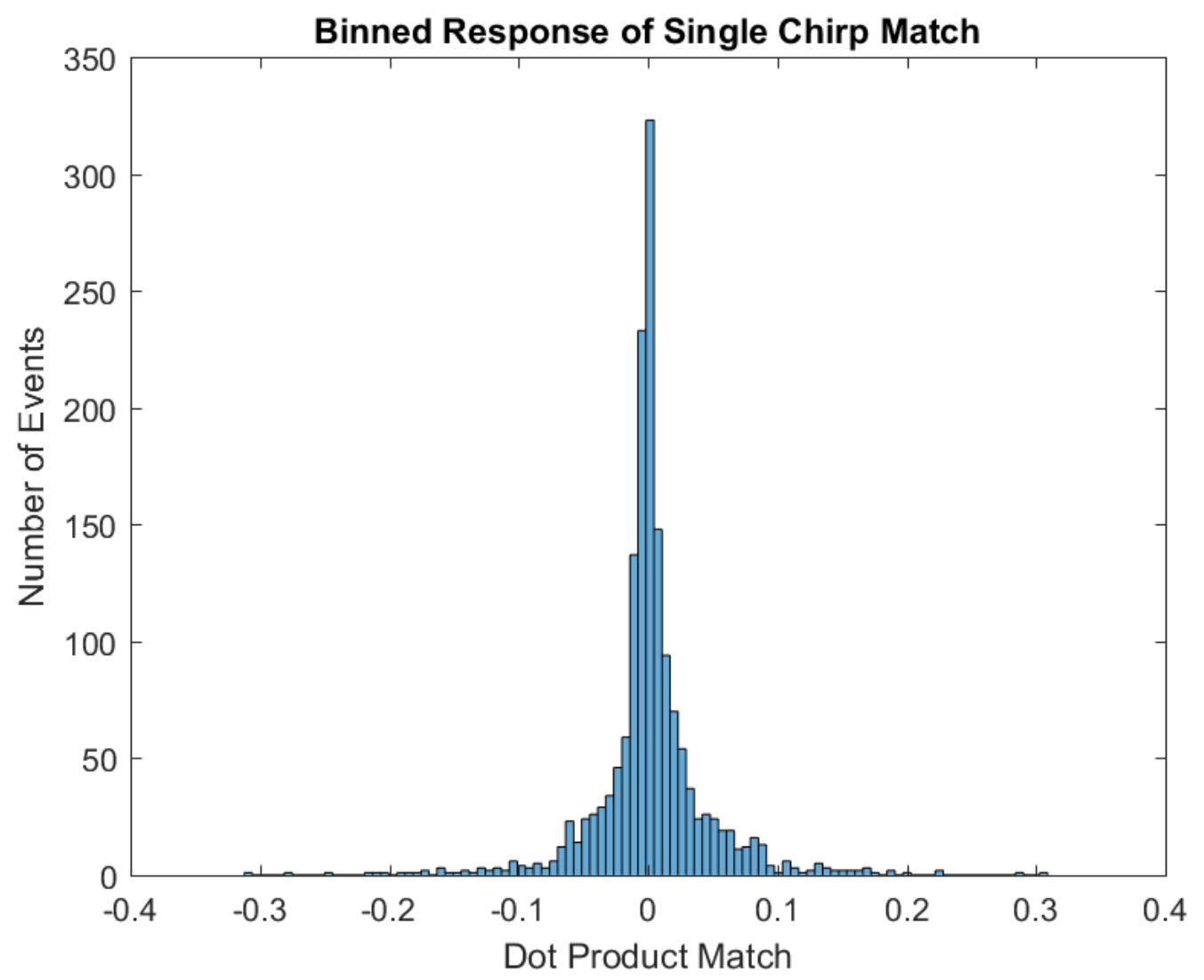

Figure 9.1: Histogram of single chirplet response. 


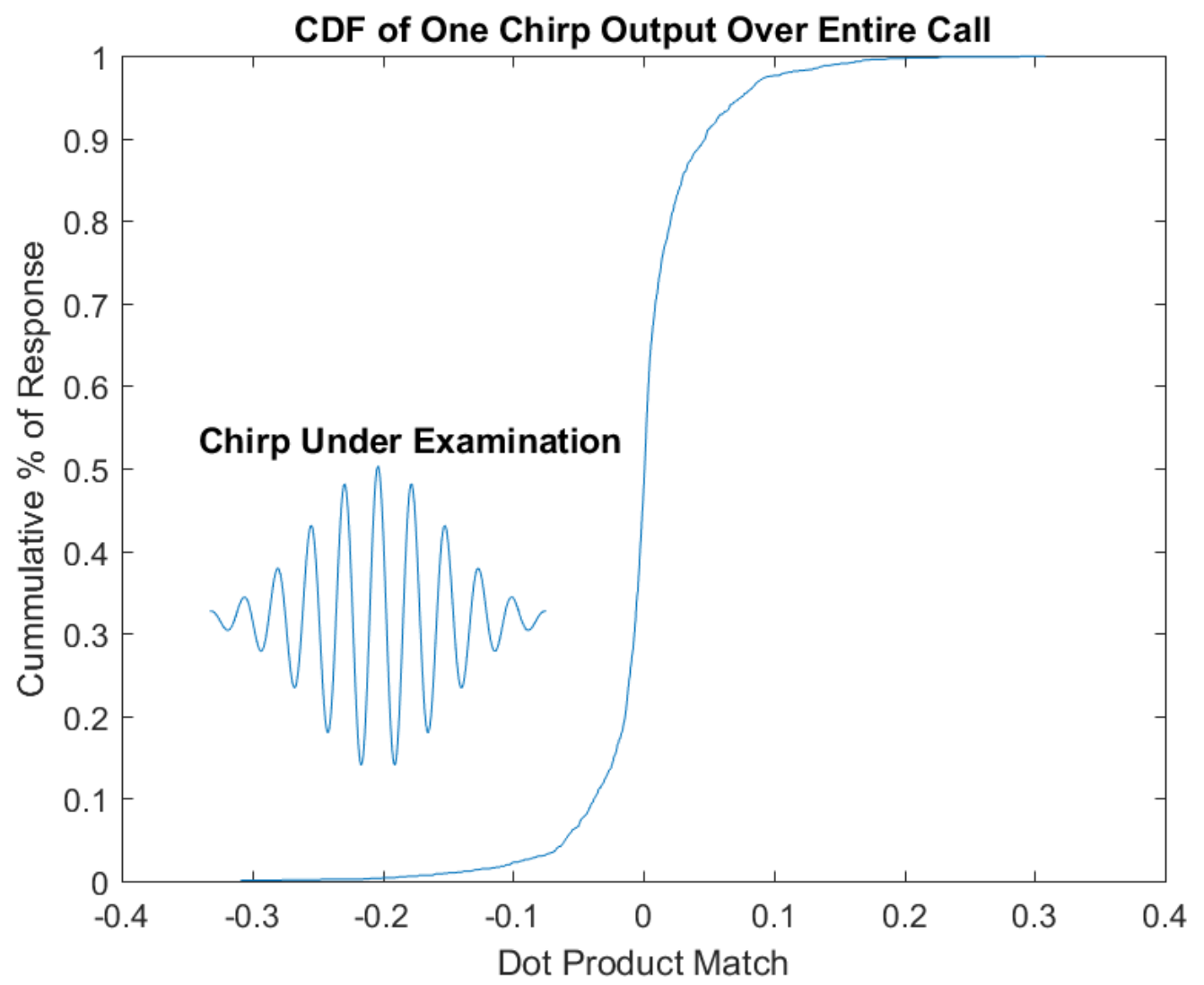

Figure 9.2: Cumulative distribution function of a particular chirp.

One other application space of interest for this project is identification of musical instruments. Given the fine details which differentiate a piano versus an oboe middle C note, musical identification seems like another excellent avenue for demonstration and evaluation of the chirplet front end.

Another area would be further examination of the proper back end machine learning structure to pair with the chirpletgram. Traditionally the convolutional neural network has been developed to identify image data, not audio data. One could attempt to take what is known of psychoacoustics and flavor the front end and back end further to detect elements identified as important to the human brain [7]. One psychoacoustic example is the importance of the harmonic structure of 
sound. The brain can fill missing harmonics when they are occluded. A modification of the chirpletgram or the machine learning back end could emphasize or detect certain harmonic pairings or structures to identify certain sounds. One could delve farther to modify the system, pairing further phsycoaccoutically relevant detectors and structured back ends. 


\section{REFERENCES CITED}

[1] M. Abadi, A. Agarwal, P. Barham, E. Brevdo, Z. Chen, C. Citro, G. S. Corrado, A. Davis, J. Dean, M. Devin, S. Ghemawat, I. J. Goodfellow, A. Harp, G. Irving, M. Isard, Y. Jia, R. Józefowicz, L. Kaiser, M. Kudlur, J. Levenberg, D. Mané, R. Monga, S. Moore, D. G. Murray, C. Olah, M. Schuster, J. Shlens, B. Steiner, I. Sutskever, K. Talwar, P. A. Tucker, V. Vanhoucke, V. Vasudevan, F. B. Viégas, O. Vinyals, P. Warden, M. Wattenberg, M. Wicke, Y. Yu, and X. Zheng. Tensorflow: Large-scale machine learning on heterogeneous distributed systems. CoRR, abs/1603.04467, 2016.

[2] M. Abadi, P. Barham, J. Chen, Z. Chen, A. Davis, J. Dean, M. Devin, S. Ghemawat, G. Irving, M. Isard, M. Kudlur, J. Levenberg, R. Monga, S. Moore, D. G. Murray, B. Steiner, P. A. Tucker, V. Vasudevan, P. Warden, M. Wicke, $\mathrm{Y}$. $\mathrm{Yu}$, and $\mathrm{X}$. Zhang. Tensorflow: A system for large-scale machine learning. CoRR, abs/1605.08695, 2016.

[3] J. A. Agamaite, C.-J. Chang, M. S. Osmanski, and X. Wang. A quantitative acoustic analysis of the vocal repertoire of the common marmoset (callithrix jacchus). The Journal of the Acoustical Society of America, 138(5):2906-2928, 2015.

[4] D. Amodei, R. Anubhai, E. Battenberg, C. Case, J. Casper, B. Catanzaro, J. Chen, M. Chrzanowski, A. Coates, G. Diamos, E. Elsen, J. Engel, L. Fan, C. Fougner, T. Han, A. Hannun, B. Jun, P. LeGresley, L. Lin, S. Narang, A. Ng, S. Ozair, R. Prenger, J. Raiman, S. Satheesh, D. Seetapun, S. Sengupta, Y. Wang, Z. Wang, C. Wang, B. Xiao, D. Yogatama, J. Zhan, and Z. Zhu. Deep speech 2: End-to-end speech recognition in english and mandarin. 2015.

[5] M. W. Ansari. Atlas of Ocular Anatomy. Cham : Springer International Publishing : Imprint: Springer, 2016.

[6] J. K. Bizley and Y. E. Cohen. The what, where and how of auditory-object perception. Nat Rev Neurosci, 14(10):693-707, 2013.

[7] A. Bregman. Auditory Scene Analysis: The Perceptual Organization of Sound. Bradford Books, 1994.

[8] C. Casebeer. A-customizable-artificial-auditory-fovea. https://github.com/ ccasebeer/A-Customizable-Artificial-Auditory-Fovea, 2018.

[9] E. C. Cherry. Some experiments on the recognition of speech, with one and with 2 ears. Journal of the Acoustical Society of America, 25(5):975-979, 1953. 
[10] L. Cohen. Time-frequency analysis. Englewood Cliffs, N.J : Prentice Hall PTR, Englewood Cliffs, N.J, 1995.

[11] T. M. Cover. Elements of information theory. New York : Wiley, New York, 1991.

[12] A. Damien. Tensorflow-examples. https://github.com/aymericdamien/ TensorFlow-Examples, 2018.

[13] B. Daniel and W. Xiaoqin. The neuronal representation of pitch in primate auditory cortex. Nature, 436(7054):1161, 2005.

[14] P. G. Franois Auger, Olivier Lemoine and P. Flandrin. Matlab Time-Frequency Toolbox. CNRS (France) and Rice University (USA), 1996.

[15] I. Goodfellow, Y. Bengio, and A. Courville. Deep Learning. The MIT Press, 2016

[16] T. Han. Around the world in 60 days: Getting deep speech to work in mandarin. 2016.

[17] A. Hannun, C. Case, J. Casper, B. Catanzaro, G. Diamos, E. Elsen, R. Prenger, S. Satheesh, S. Sengupta, A. Coates, and A. Y. Ng. Deep speech: Scaling up end-to-end speech recognition. 2014.

[18] N. Kishi, K. Sato, E. Sasaki, and H. Okano. Common marmoset as a new model animal for neuroscience research and genome editing technology. Development, Growth \& Differentiation, 56(1):53-62, 2014.

[19] C. Koppl, O. Gleich, and G. Manley. An auditory fovea in the barn owl cochlea. Sensory, Neural and Behavioral Physiology, 171(6):695-704, 1993.

[20] L. Liang, T. Lu, and X. Q. Wang. Neural representations of sinusoidal amplitude and frequency modulations in the primary auditory cortex of awake primates. Journal of Neurophysiology, 87(5):2237-2261, 2002.

[21] T. Lu, L. Liang, and X. Q. Wang. Temporal and rate representations of timevarying signals in the auditory cortex of awake primates. Nature Neuroscience, 4(11):1131-1138, 2001.

[22] R. Lyons. Understanding Digital Signal Processing. Pearson Education, 2010.

[23] S. Mann and S. Haykin. The chirplet transform: A generalization of gabors logon transform. 1991.

[24] S. Mann and S. Haykin. Chirplet transform: physical considerations. IEEE Transactions on Signal Processing, 43(11):2745-2761, 1995. 
[25] MATLAB. MATLAB Statistics and Machine Learning Toolbox Version Version 11.1 (R2017a). The MathWorks Inc., Natick, Massachusetts, 2017.

[26] MATLAB. MATLAB Version 9.2.0.556344 (R2017a). The MathWorks Inc., Natick, Massachusetts, 2017.

[27] C. T. Miller, W. A. Freiwald, D. A. Leopold, J. F. Mitchell, A. C. Silva, and X. Wang. Marmosets: A neuroscientific model of human social behavior. Neuron, 90(2):219-233, 2016.

[28] E. C. Mora, S. Macias, J. Hechavarria, M. Vater, and M. Koessl. Evolution of the heteroharmonic strategy for target-range computation in the echolocation of mormoopidae. Frontiers in Physiology, 4, 2013.

[29] A. Nguyen, J. Yosinski, and J. Clune. Deep neural networks are easily fooled: High confidence predictions for unrecognizable images. 2014.

[30] A. Oppenheim and R. Schafer. Discrete-Time Signal Processing. Pearson Education, 2011.

[31] A. Oppenheim, A. Willsky, and S. Nawab. Signals \& Systems. Prentice-Hall International, 1997.

[32] F. Pedregosa, G. Varoquaux, A. Gramfort, V. Michel, B. Thirion, O. Grisel, M. Blondel, P. Prettenhofer, R. Weiss, V. Dubourg, J. Vanderplas, A. Passos, D. Cournapeau, M. Brucher, M. Perrot, and E. Duchesnay. Scikit- learn: Machine learning in python. Journal Of Machine Learning Research, 12:2825$2830,2011$.

[33] M. E. Pennesi, A. K. Garg, S. Feng, K. V. Michaels, T. B. Smith, J. D. Fay, A. R. Weiss, L. M. Renner, S. Hurst, T. J. McGill, A. Cornea, K. D. Rittenhouse, M. Sperling, J. Fruebis, and M. Neuringer. Measuring Cone Density in a Japanese Macaque (Macaca fuscata) Model of Age-Related Macular Degeneration with Commercially Available Adaptive Optics, pages 309-316. Springer New York, New York, NY, 2014.

[34] H.-U. Schnitzler and A. Denzinger. Auditory fovea and doppler shift compensation: adaptations for flutter detection in echolocating bats using cf-fm signals. Neuroethology, Sensory, Neural, and Behavioral Physiology, 197(5):541$559,2011$.

[35] G. Schuller and G. Pollak. Disproportionate frequency representation in the inferior colliculus of doppler-compensating greater horseshoe bats: Evidence for an acoustic fovea. Journal of comparative physiology, 132(1):47-54, 1979.

[36] J. Smith. Mathematics of the Discrete Fourier Transform (DFT): With Audio Applications. BookSurge Publishing, 2007. 
[37] J. Smith, S. U. C. f. C. R. i. Music, Acoustics, and S. U. D. o. Music. Spectral Audio Signal Processing. W3K, 2011.

[38] N. Srivastava, G. Hinton, A. Krizhevsky, I. Sutskever, and R. Salakhutdinov. Dropout: A simple way to prevent neural networks from overfitting. Journal of Machine Learning Research, 15:1929-1958, 2014.

[39] J. Su, D. Vasconcellos Vargas, and S. Kouichi. One pixel attack for fooling deep neural networks. ArXiv e-prints, 1710:arXiv:1710.08864, 2017.

[40] H. K. Turesson, S. Ribeiro, D. R. Pereira, J. P. Papa, and V. H. C. de Albuquerque. Machine learning algorithms for automatic classification of marmoset vocalizations. Plos One, 11(9), 2016.

[41] F. V. VEEN. The neural network zoo, 2016.

[42] B. Wandell. Foundations of Vision. Sinauer Associates, 1995.

[43] L. Yann, B. Yoshua, and H. Geoffrey. Deep learning. Nature, 521(7553):436, 2015. 


\section{APPENDIX: MATLAB ARTIFICIAL ACCOUSIC FOVEA CHIRP AND DECK}

\section{CREATION}

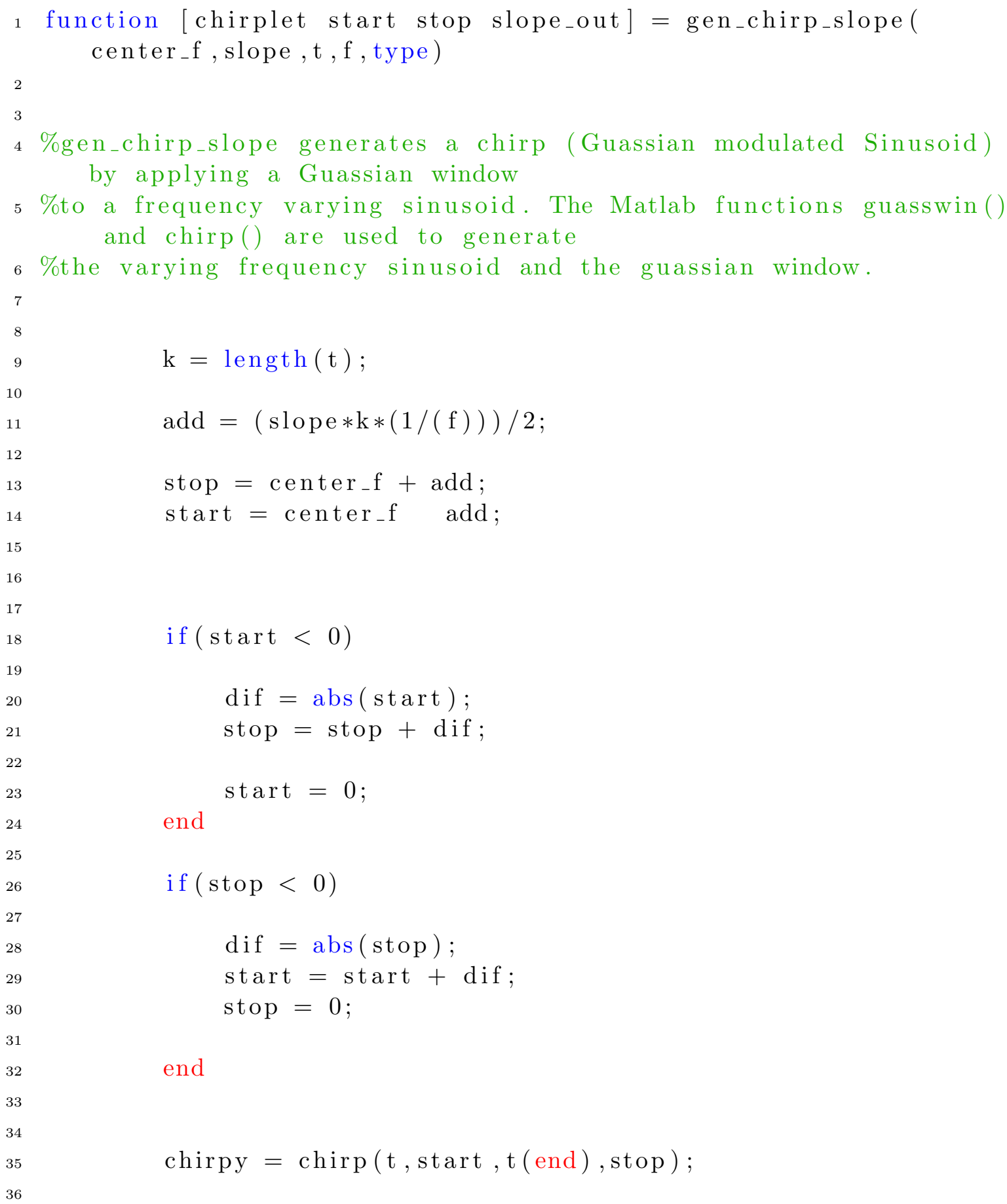


65

67

68

69

70

71

72

73

74

$75 \% a l 1 \_d e s c$

$76 \%$ Description of every chirplet waveform

$77 \%$ Columns as follows.

${ }_{78} \% 1$ Unique Identifier based on slope and length but not frequency

location if $(\operatorname{strcmp}($ type, 'gauss'))

let $=$ gausswin $(\text { length }(\operatorname{chirpy}))^{\prime}$;

elseif ( $\operatorname{strcmp}($ type, ' up_lin'))

let $=$ linspace $\left(0,1\right.$, length $\left(\right.$ chirpy $\left.\left.^{\prime}\right)\right)$;

elseif (strcmp(type, 'down_lin'))

let $=$ linspace $\left(1,0\right.$, length $\left(\right.$ chirpy $\left.\left.^{\prime}\right)\right)$;

els e

return

end

slope_out $=($ stop start $) /(\mathrm{k} / \mathrm{f})$

chirplet $=$ chirpy.$*$ let; function [all_chirps all_desc freq_slopes t window_length_ts chirp_type_key deck_param] = chirplets_f(deck_param)

\%chirplets_f creates all the chirplets comprising a deck given the deck parameters passed in deck_param.

$\%$ Deck_Param

\%Structure defining the chirplet deck.

\%all_chirps

\%The actual sampled chirps.

$\%$ N time cell arrays of all the actual chirplet time waveforms. 
79

88

89

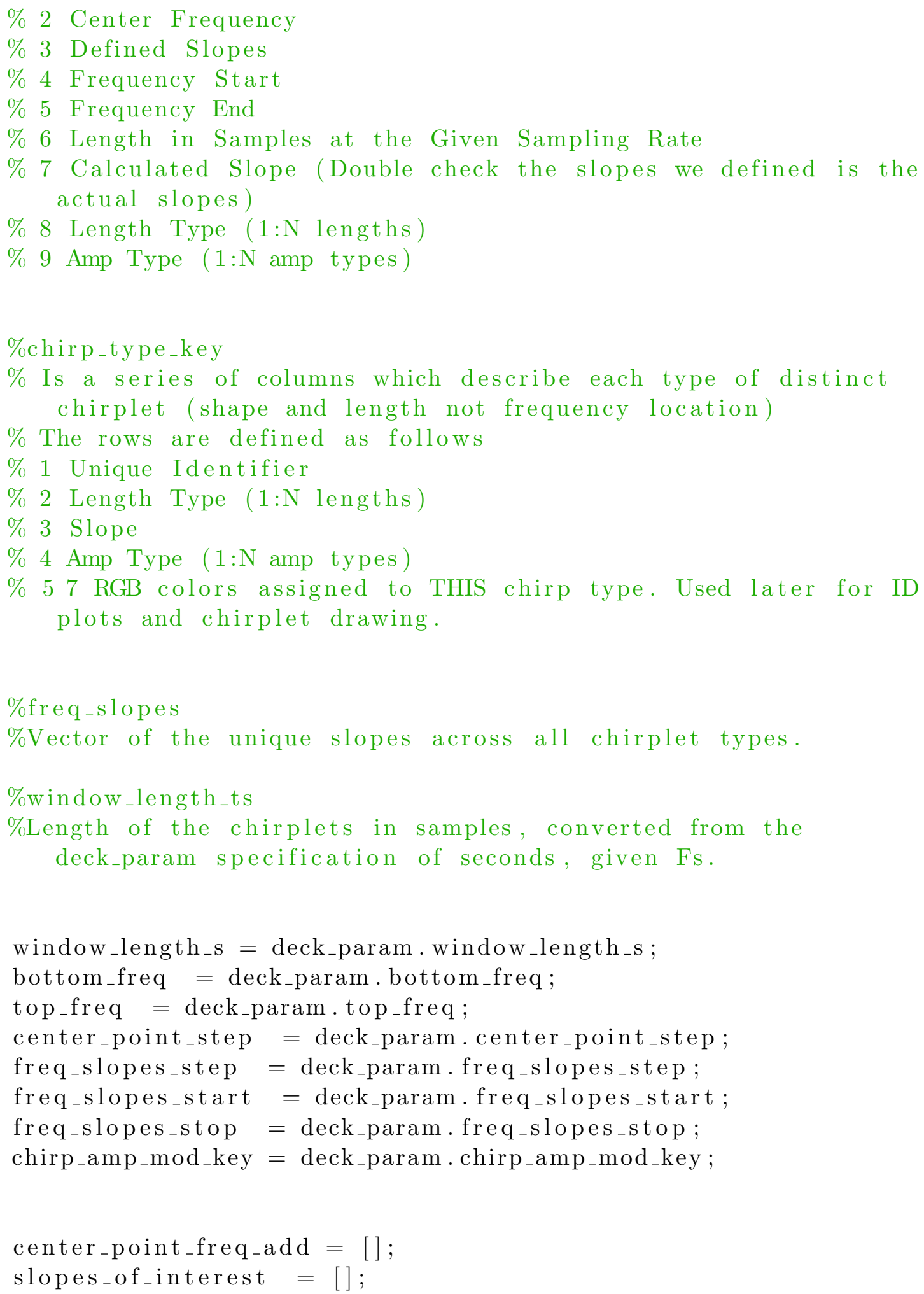


160

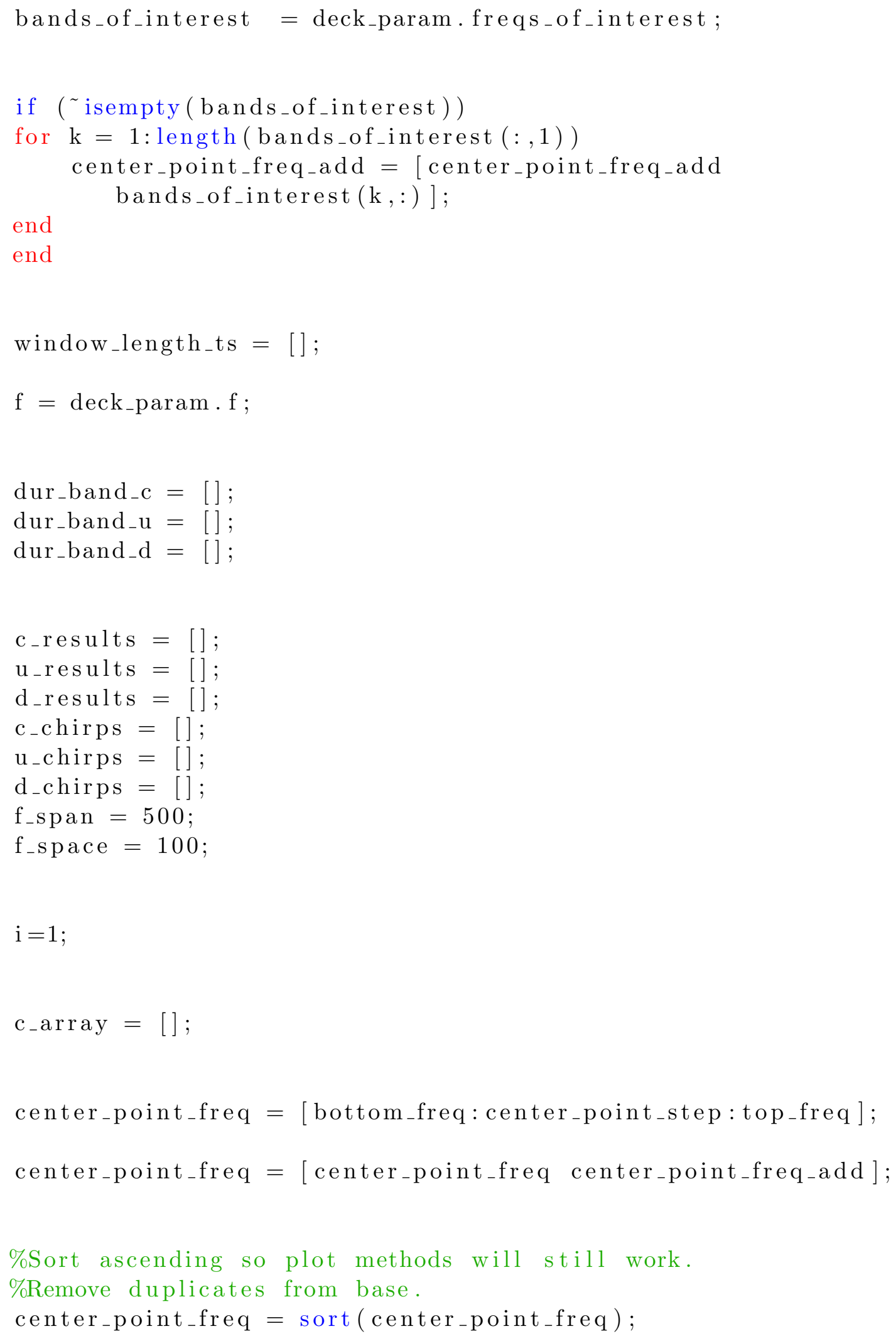


201

202

203

204

205

206

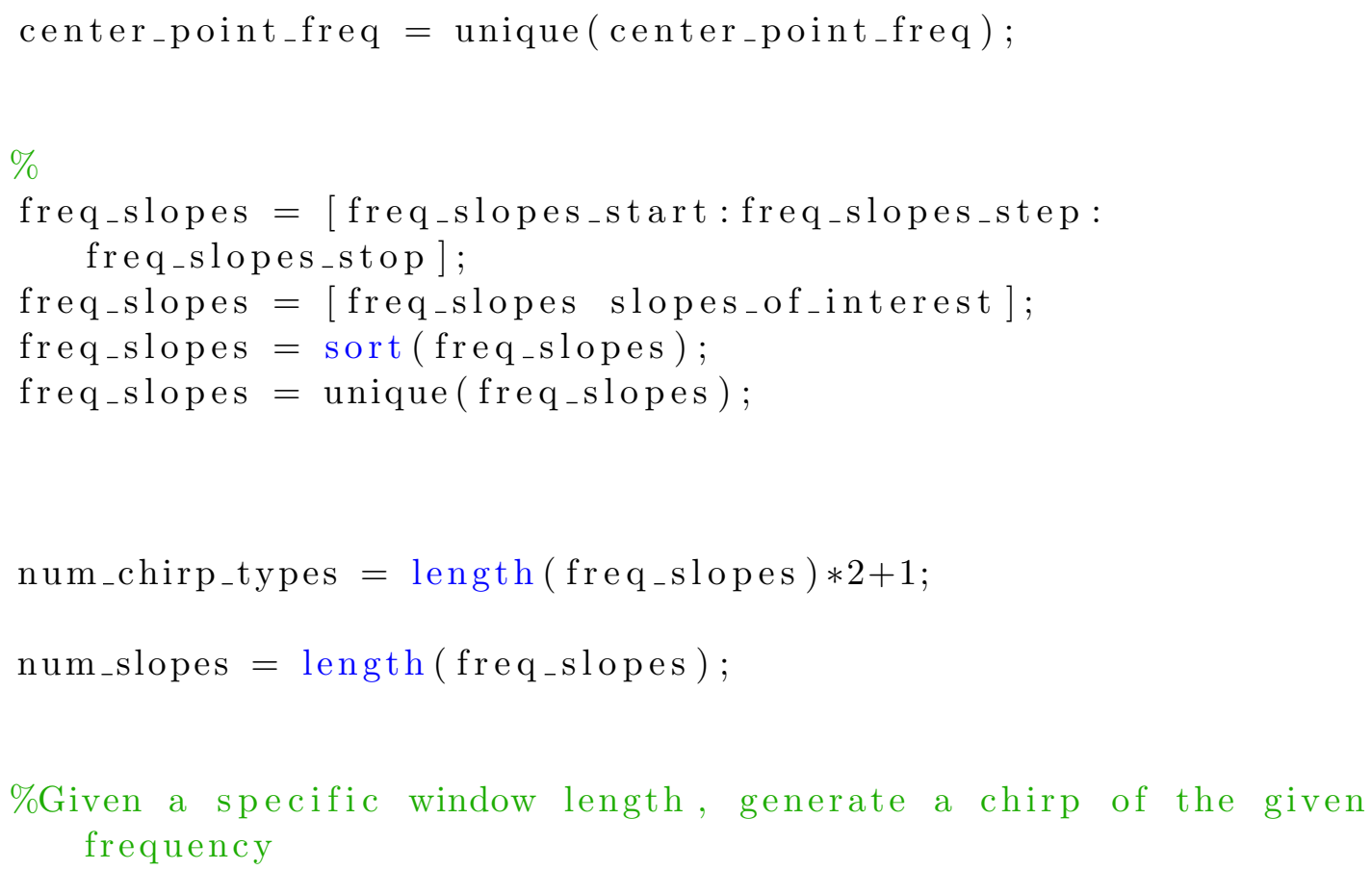




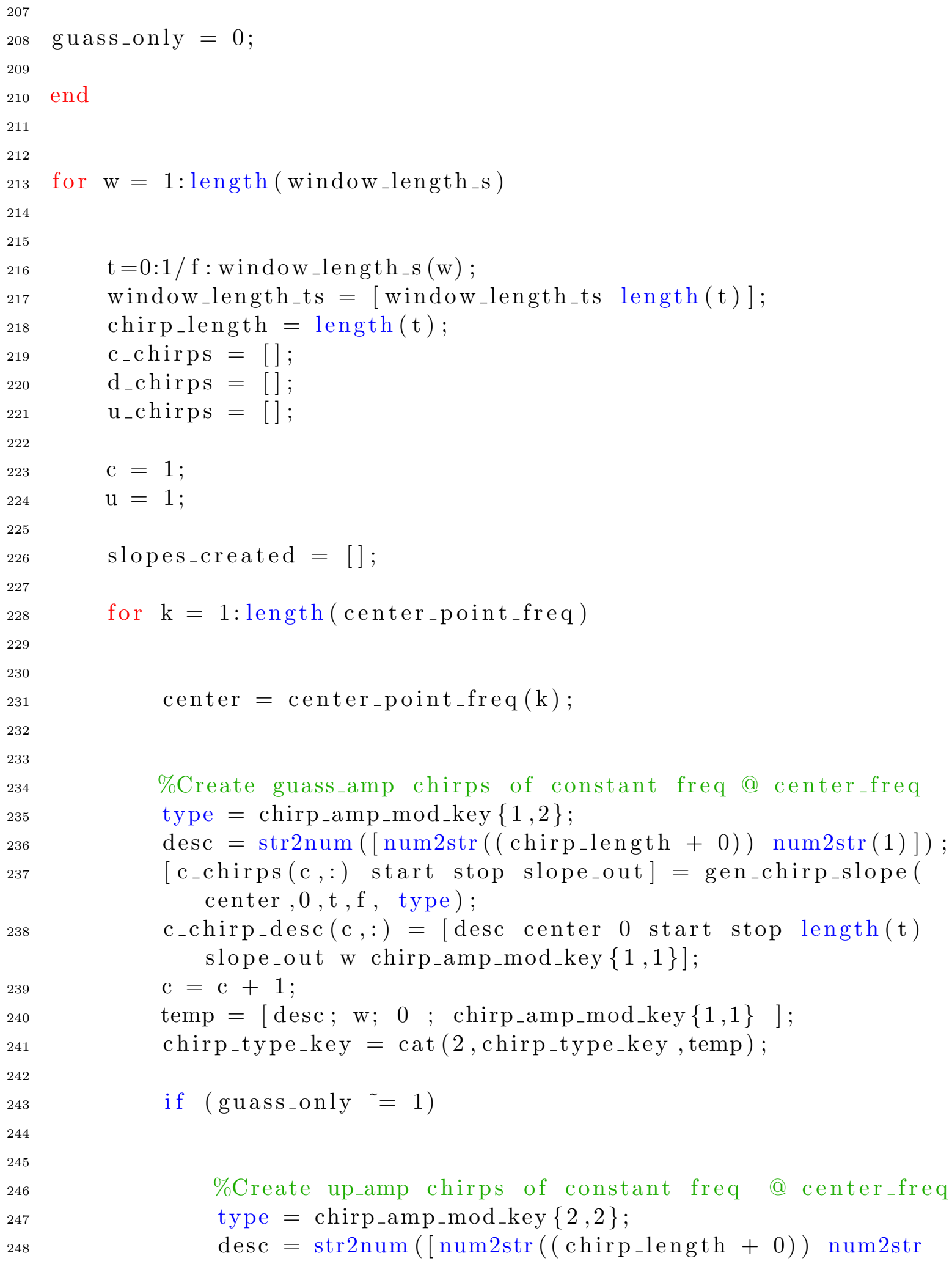




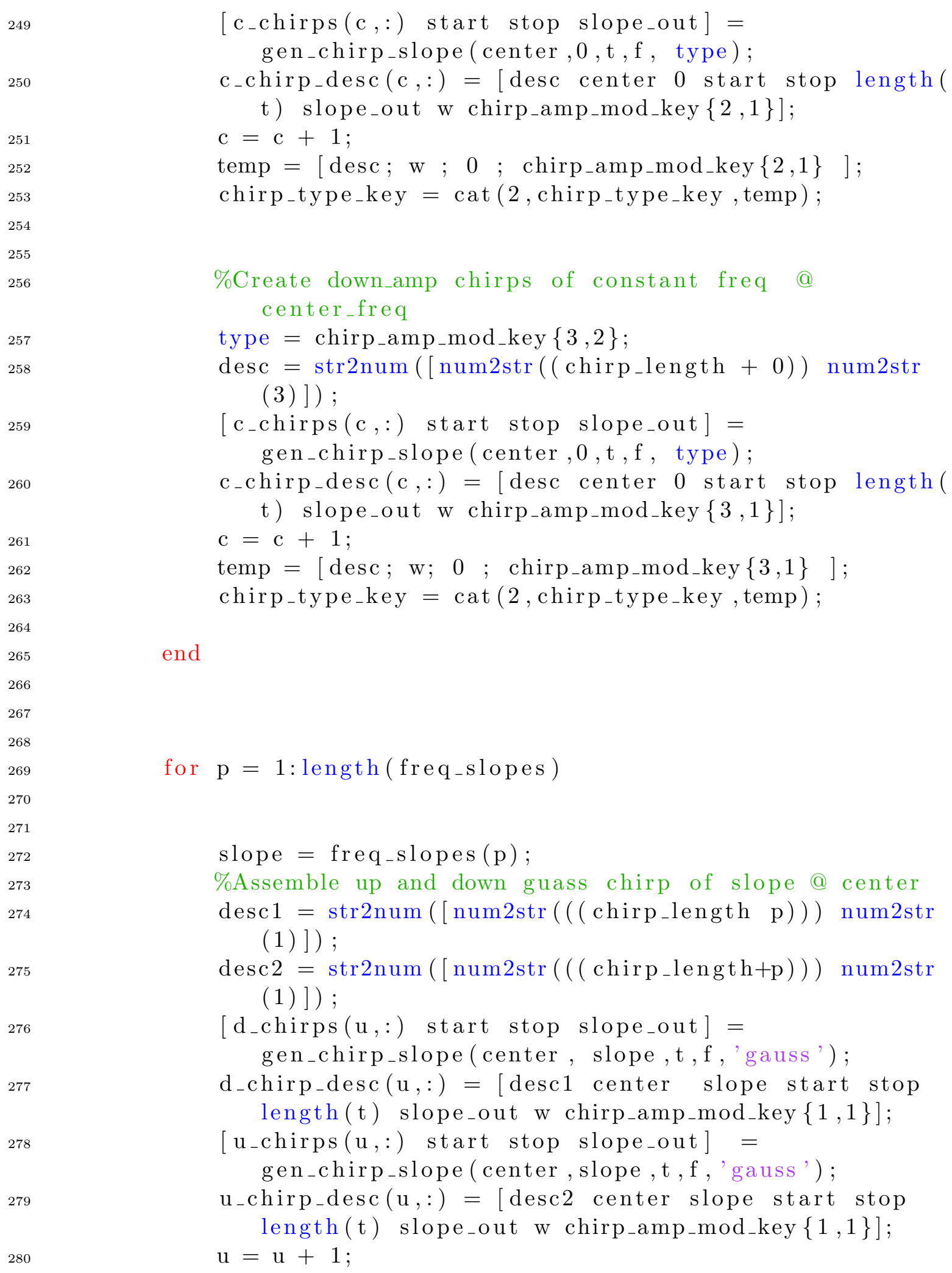

[ $c_{-}$chirps $(\mathrm{c},:)$ start stop slope_out] $=$ gen_chirp_slope (center, $0, \mathrm{t}, \mathrm{f}$, type); $c_{-}$chirp_desc $(c,:)=[$ desc center 0 start stop length ( t) slope_out w chirp_amp_mod_key $\{2,1\}]$;

$\mathrm{c}=\mathrm{c}+1$;

temp $=\left[\right.$ desc $; \mathrm{w} ; 0 ; \operatorname{chirp}{ }_{-}$amp_mod_key $\left.\{2,1\}\right]$; chirp_type_key $=$ cat $(2$, chirp_type_key, temp $)$;

\%Create down_amp chirps of constant freq @ center_freq

type $=$ chirp_amp_mod_key $\{3,2\}$;

desc $=\operatorname{str} 2$ num $([$ num $2 \operatorname{str}(($ chirp_length +0$))$ num 2 str (3) ]) ;

[ $\mathrm{c}_{-} \mathrm{chirps}(\mathrm{c},:)$ start stop slope - out $]=$ gen_chirp_slope (center, $0, \mathrm{t}, \mathrm{f}$, type);

$c_{-}$chirp_desc $(c,:)=[$ desc center 0 start stop length (

t) slope_out w chirp_amp_mod_key $\{3,1\}]$;

$\mathrm{c}=\mathrm{c}+1$

temp $=[$ desc $; \mathrm{w} ; 0 ;$ chirp_amp_mod_key $\{3,1\}]$;

chirp_type_key $=$ cat $(2$, chirp_type_key, temp $)$;

end

for $p=1: \operatorname{length}($ freq-slopes $)$

slope $=$ freq-slopes $(\mathrm{p})$;

\%Assemble up and down guass chirp of slope@ center $\operatorname{desc} 1=\operatorname{str} 2$ num $([$ num $2 \operatorname{str}((($ chirp_length p $)))$ num 2 str (1) ] ) ;

desc2 $=\operatorname{str} 2$ num $([$ num $2 \operatorname{str}((($ chirp_length + p $)))$ num 2 str (1) ]);

$\left[d_{-}\right.$chirps $(u,:)$ start stop slope_out] $=$ gen_chirp_slope (center, slope,t,f, 'gauss') ;

$d_{-}$chirp_desc $(u,:)=[$ desc1 center slope start stop length(t) slope_out w chirp_amp_mod_key $\{1,1\}]$; $\left[u_{-}\right.$chirps $(u,:)$ start stop slope_out] = gen_chirp_slope (center, slope,t, f, 'gauss') ; $u_{-}$chirp_desc $(u,:)=[$ desc2 center slope start stop length(t) slope_out w chirp_amp_mod_key $\{1,1\}]$; $\mathrm{u}=\mathrm{u}+1$; 
temp $=[\operatorname{desc} 1 \operatorname{desc} 2 ; \mathrm{w}$ w; 0 0; chirp_amp_mod_key $\{1,1\}$ chirp_amp_mod_key $\{1,1\} \quad]$; chirp_type_key $=$ cat $(2$, chirp_type_key,temp $)$;

if (guass_only $\sim 1$ )

\%Assemble up and down up lin chirp of slope @ center

slope $=$ freq-slopes $(\mathrm{p})$;

type $=$ chirp_amp_mod_key $\{2,2\}$;

$\operatorname{desc} 1=\operatorname{str} 2 \operatorname{num}\left(\left[\operatorname{num} 2 \operatorname{str}\left(\left(\left(\operatorname{chirp} \_\right.\right.\right.\right.\right.$length $\left.\left.\left.p\right)\right)\right)$ num $2 \operatorname{str}(2)])$;

$\operatorname{desc} 2=\operatorname{str} 2 \operatorname{num}\left(\left[\operatorname{num} 2 \operatorname{str}\left(\left(\left(\operatorname{chir} p_{-}\right.\right.\right.\right.\right.$length $\left.\left.\left.+\mathrm{p}\right)\right)\right)$ num $2 \operatorname{str}(2)])$;

$\left[d_{-}\right.$chirps(u,:) start stop slope_out] $=$ gen_chirp_slope (center, slope,t, f, type);

$d_{-}$chirp_desc $(u,:)=[$ desc1 center slope start stop length(t) slope_out w chirp_amp_mod_key $\{2,1\}]$;

$\left[u_{-}\right.$chirps $(u,:)$ start stop slope_out $]=$ gen_chirp_slope (center, slope,t,f,type);

$\mathrm{u}_{-}$chirp_desc $(\mathrm{u},:)=[\operatorname{desc} 2$ center slope start stop length(t) slope_out w chirp_amp_mod_key $\{2,1\}]$;

$\mathrm{u}=\mathrm{u}+1$

temp $=[\operatorname{desc} 1$ desc $2 ; \mathrm{w}$ w; 0 0; chirp_amp_mod_key $\{2,1\}$ chirp_amp_mod_key $\{2,1\} \quad]$;

chirp_type_key $=$ cat $(2$, chirp_type_key, temp $)$;

\%Assemble up and down up down chirp of slope@ center

slope $=$ freq-slopes $(p) ;$

type $=$ chirp_amp_mod_key $\{3,2\}$;

$\operatorname{desc} 1=\operatorname{str} 2 \operatorname{num}\left(\left[\operatorname{num} 2 \operatorname{str}\left(\left(\left(\operatorname{chirp} \_\right.\right.\right.\right.\right.$length p $\left.\left.)\right)\right)$ $\operatorname{num} 2 \operatorname{str}(3)])$;

$\operatorname{desc} 2=\operatorname{str} 2 \operatorname{num}\left(\left[\operatorname{num} 2 \operatorname{str}\left(\left(\left(\operatorname{chirp} \operatorname{plength}_{\mathrm{p}} \mathrm{p}\right)\right)\right)\right.\right.$ num $2 \operatorname{str}(3)])$;

$\left[d_{-}\right.$chirps $(u,:)$ start stop slope_out] $=$ gen_chirp_slope (center, slope,t,f,type);

$d_{-}$chirp_desc $(u,:)=[$ desc1 center slope start stop length(t) slope_out w chirp_amp_mod_key $\{3,1\}]$;

[u_chirps(u,:) start stop slope_out]= 
322

323

324

325

326

327

328

329

330

331

332

333

334

335

336

337

338

339

340

341

342

343

344

345

346

347

348

349

350 gen_chirp_slope (center, slope,t,f,type); $u_{-}$chirp_desc $(u,:)=[\operatorname{desc} 2$ center slope start stop length(t) slope_out w chirp_amp_mod_key $\{3,1\}]$;

$\mathrm{u}=\mathrm{u}+1$;

temp $=[\operatorname{desc} 1$ desc $2 ; \mathrm{w}$ w; 0 0; chirp_amp_mod_key

$\{3,1\}$ chirp_amp_mod_key $\{3,1\} \quad]$;

chirp_type_key $=$ cat $(2$, chirp_type_key, temp $)$;

end

end

end

$\mathrm{c}_{-} \mathrm{chirps}_{-} \operatorname{cell}\{\mathrm{w}\}=\mathrm{c}_{-}$chirps;

$\mathrm{d}_{-}$chirps_cell $\{\mathrm{w}\}=\mathrm{d}_{-}$chirps;

$\mathrm{u}_{-} \mathrm{chirps} \mathrm{s}_{-} \operatorname{cell}\{\mathrm{w}\}=\mathrm{u}_{-} \mathrm{chirps}$

all_chirps_cell $\{\mathrm{w}\}=\left[\mathrm{c}_{-} \mathrm{chirps} ; \mathrm{d}_{-}\right.$chirps; u_chirps $]$;

all_desc_cell $\{\mathrm{w}\}=\left[\mathrm{c}_{-}\right.$chirp_desc; d_chirp_desc; $\left.\mathrm{u}_{-} \mathrm{chir} \mathrm{p}_{-} \mathrm{desc}_{-}\right]$; end

all_chirps $=$ all_chirps_cell $_{-}$

all_desc $=[]$

for $k=1: \operatorname{length}($ all_desc_cell $)$

all_desc $=[$ all_desc $;$ all_desc_cell $\{\mathrm{k}\}]$

end

chirp_type_key $=$ unique( chirp_type_key', 'rows') '; 


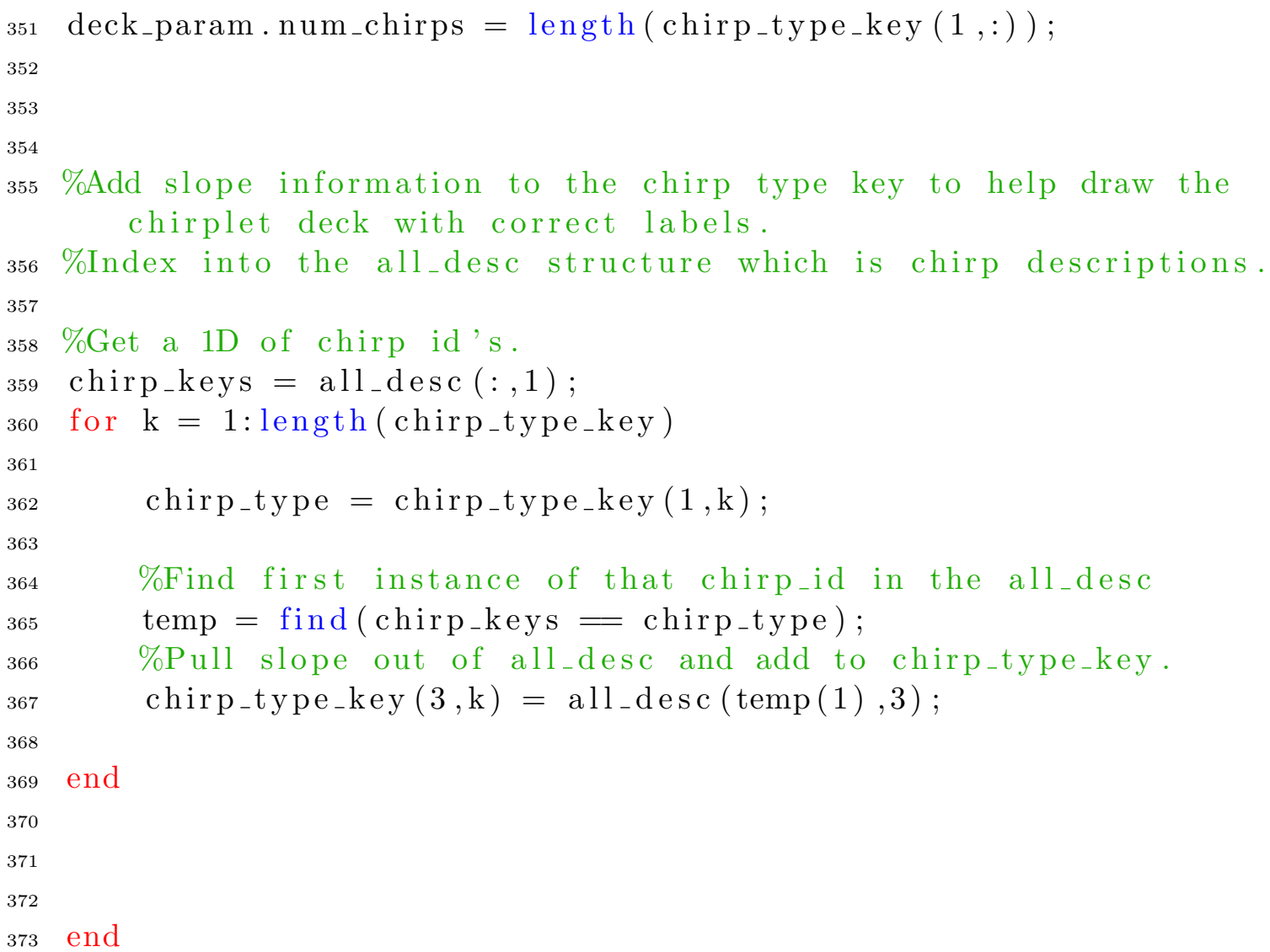

